LBNL-52216

\title{
Instrumented Home Energy Rating and Commissioning
}

\author{
Craig Wray, Iain Walker, Max Sherman
}

\author{
Environmental Energy Technologies Division \\ Indoor Environment Department \\ Lawrence Berkeley National Laboratory \\ Berkeley, CA 94720
}

May 2003

This report describes work supported by the California Energy Commission through the Public Interest Energy Research program under contract no. 500-98-033, and by the Assistant Secretary for Energy Efficiency and Renewable Energy, Building Technologies Program, of the U.S. Department of Energy under contract no. DEAC03-76SF00098. 
The Lawrence Berkeley National Laboratory, 1 Cyclotron Road, Berkeley, CA 94720 is a national laboratory of the U.S. Department of Energy managed by the Regents of the University of California for the U.S. Department of Energy under Contract Number DEAC03-76SF00098. This report was prepared as a result of work sponsored by the California Energy Commission (Commission). It does not necessarily represent the views of the Commission, its employees, or the State of California. The Commission, the State of California, its employees, contractors, and subcontractors make no warranty, express or implied, and assume no legal liability for the information in this report, nor does any party represent that the use of this information will not infringe upon privately owned rights. This report has not been approved or disapproved by the Commission nor has the Commission passed upon the accuracy or adequacy of the information in this report.

THE GOVERNMENT AND THE CONTRACTOR MAKE NO EXPRESS OR IMPLIED WARRANTY AS TO THE CONDITIONS OF THE RESEARCH OR ANY INTELLECTUAL PROPERTY, GENERATED INFORMATION, OR PRODUCT MADE OR DEVELOPED UNDER THIS AGREEMENT, OR THE OWNERSHIP, MERCHANTABILITY, OR FITNESS FOR A PARTICULAR PURPOSE OF THE RESEARCH OR RESULTING PRODUCT; THAT THE GOODS, SERVICES, MATERIALS, PRODUCTS, PROCESSES, INFORMATION, OR DATA TO BE FURNISHED HEREUNDER WILL ACCOMPLISH INTENDED RESULTS OR ARE SAFE FOR ANY PURPOSE INCLUDING THE INTENDED PURPOSE; OR THAT ANY OF THE ABOVE WILL NOT INTERFERE WITH PRIVATELY OWNED RIGHTS OF OTHERS. NEITHER THE GOVERNMENT NOR THE CONTRACTOR SHALL BE LIABLE FOR SPECIAL, CONSEQUENTIAL, OR INCIDENTAL DAMAGES ATTRIBUTED TO SUCH RESEARCH OR RESULTING PRODUCT, INTELLECTUAL PROPERTY, GENERATED INFORMATION, OR PRODUCT MADE OR DELIVERED UNDER THIS AGREEMENT. 


\section{TABLE OF CONTENTS}

ABSTRACT ................................................................................................................................... 1

EXECUTIVE SUMMARY ........................................................................................................... 2

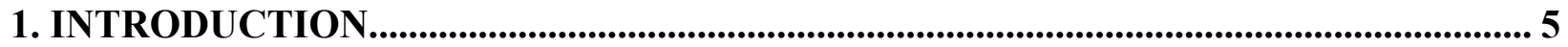

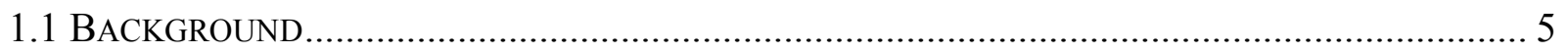

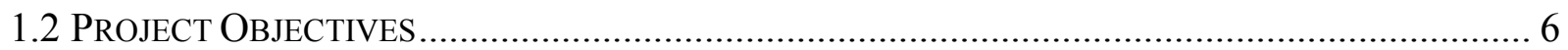

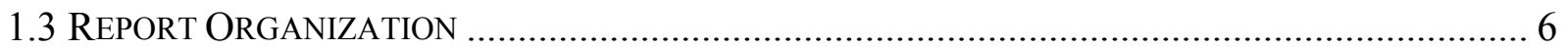

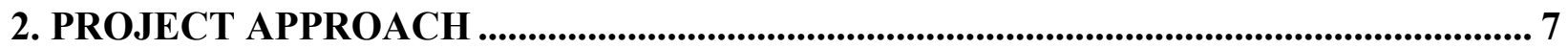

3. PROJECT OUTCOMES............................................................................................................. 8

4. CONCLUSIONS AND RECOMMENDATIONS.................................................................. 12

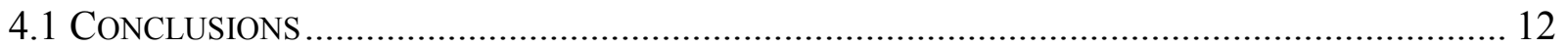

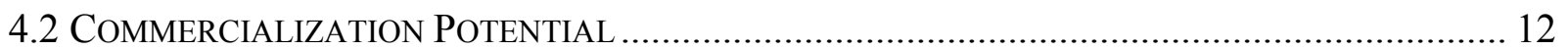

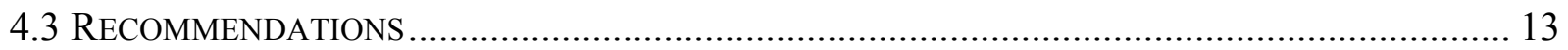

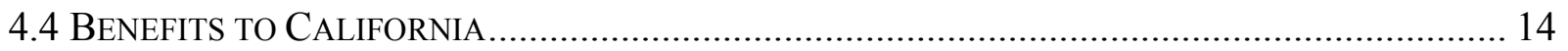

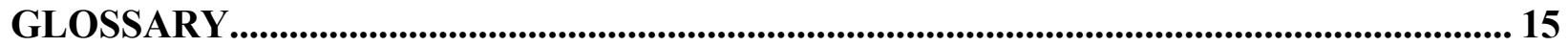

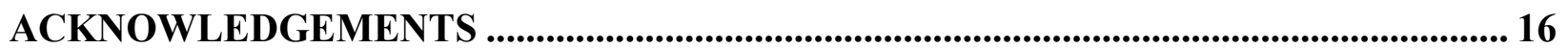

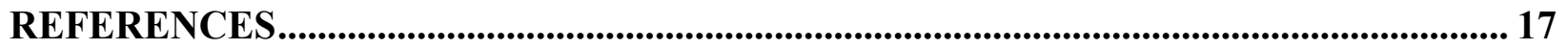

APPENDIX I. GUIDELINES FOR RESIDENTIAL COMMISSIONING...............................19

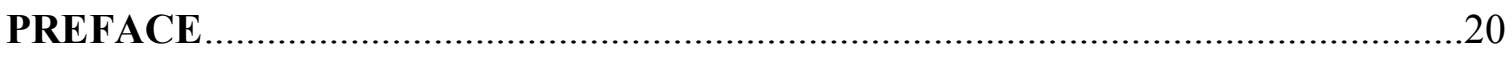

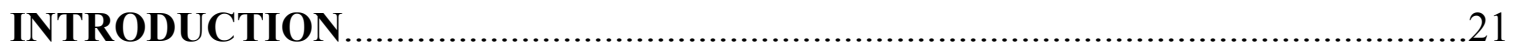

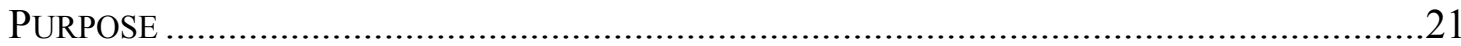

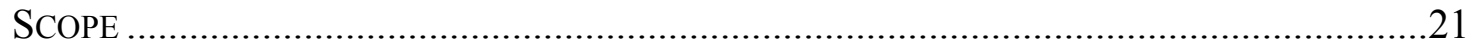

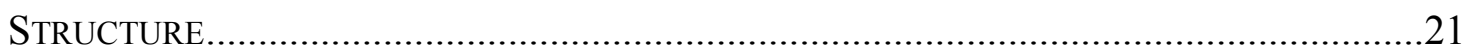

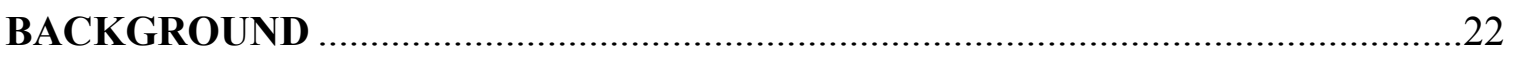

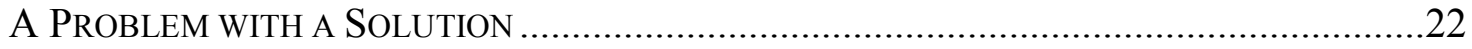

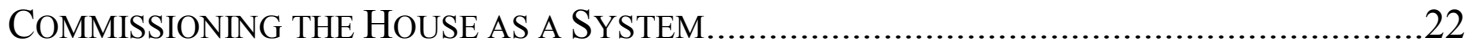

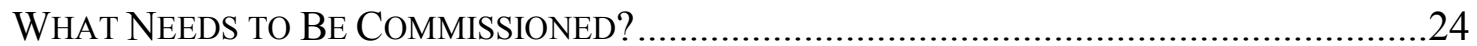

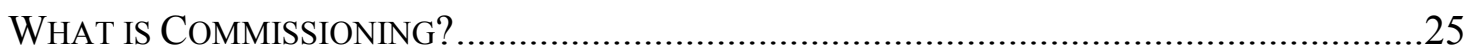

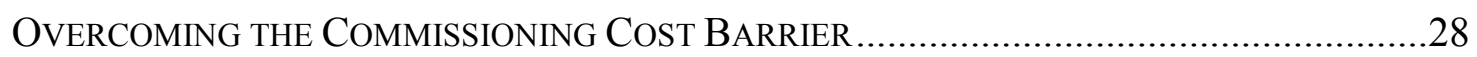

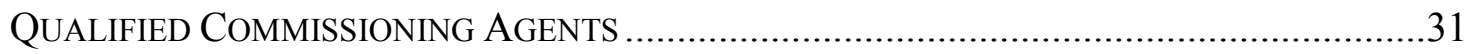

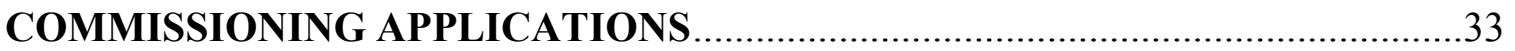

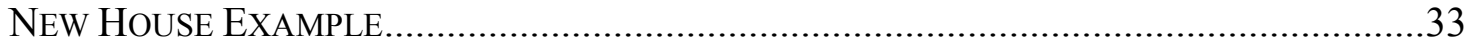

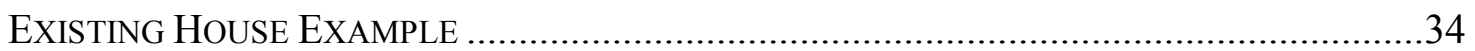

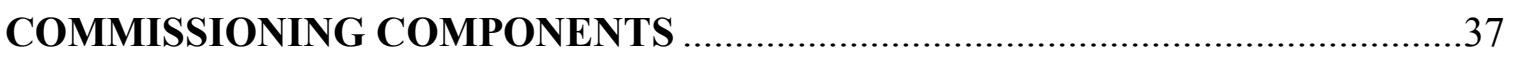

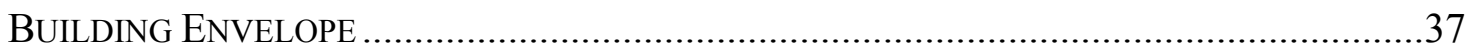




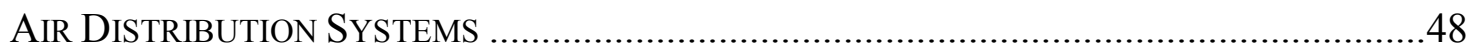

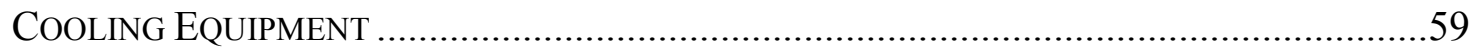

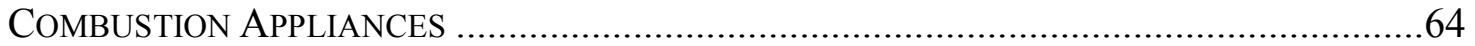

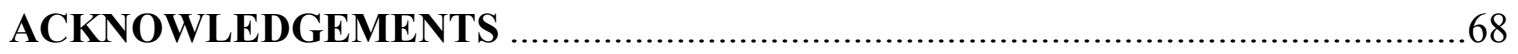

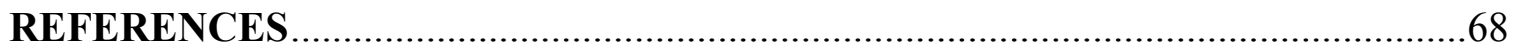

APPENDIX II. EXAMPLE AUDIT AND DIAGNOSTIC PROCESS ...............................74

APPENDIX III. EXAMPLE COMMISSIONING REPORT ...............................................82 


\section{ABSTRACT}

Currently, houses do not perform optimally or even as many codes and forecasts predict, largely because they are field assembled and there is no consistent process to identify deficiencies or to correct them. Solving this problem requires field performance evaluations using appropriate and agreed upon procedures in the form of a new process called residential commissioning. The purpose of this project is to develop and document these procedures and to demonstrate the value that applying them could provide in both new and existing California houses. This project has four specific objectives: to develop metrics and diagnostics for assessing house performance, to provide information on the potential benefits of commissioning using a whole-house approach, to develop programmatic guidelines for commissioning, and to conduct outreach efforts to transfer project results to industry stakeholders. The primary outcomes from this project are the development of residential commissioning guidelines and the analytical confirmation that there are significant potential benefits associated with commissioning California houses, particularly existing ones. In addition, we have made substantial advances in understanding the accuracy and usability of diagnostics for commissioning houses. In some cases, we have been able to work with equipment manufacturers to improve these aspects of their diagnostic tools. These outcomes provide a solid foundation on which to build a residential commissioning program in California. We expect that a concerted effort will be necessary to integrate such a program with existing building industry efforts and to demonstrate its use in the field.

Keywords: Benefits, buildings, commissioning, diagnostics, energy, HVAC, metrics, retrofits. 


\section{EXECUTIVE SUMMARY}

Introduction. Currently, houses do not perform optimally or even as many codes and forecasts predict, largely because they are field assembled and there is no consistent process to identify deficiencies or to correct them. Solving this problem in new and existing houses requires field performance evaluations using appropriate and agreed upon procedures. Many procedural elements to test the energy performance of energy-related components and systems of a home and to assess their impacts on health and safety already exist in a fragmented environment; some are ready now to integrate into a new process called residential commissioning.

Purpose. The purpose of this project is to develop and document residential commissioning procedures and to demonstrate the value that applying these procedures could provide in both new and existing California houses. We expect that this new information will lay the groundwork for a residential commissioning industry in California, which ultimately will increase the use of commissioning for California houses. In turn, we expect that the application of commissioning by people such as home energy raters, home inspectors, auditors, and weatherization contractors will improve or even optimize residential energy and indoor environmental performance, and will improve the energy cost/value of electricity for the state.

Project Objectives. The specific technical objectives for this project were to:

1. Define, assess, and articulate metrics and diagnostics ("yardsticks" and measurement techniques) for residential commissioning, with a focus on delineating them based upon their accuracy and usability in the field.

2. Provide information on the potential benefits of commissioning California houses using a whole-house approach, which recognizes that a house is a system of interacting components.

3. Develop programmatic guidelines for commissioning new and existing California houses.

4. Conduct outreach efforts to transfer project results, such as the residential commissioning guidelines, to industry stakeholders who will use the results of the work in program, code, standard, and technology development.

Project Outcomes: Based on the project objectives, the project has four primary outcomes:

Metrics \& Diagnostics. We identified and described metrics for use in residential commissioning, along with a consolidated set of 24 practical diagnostics that the building industry can use now to evaluate them (Wray et al. 2002a). This set focuses on areas of particular concern where there are significant component and system interactions: envelope insulation quality, windows, airtightness, envelope moisture, HVAC fan and duct system airflows, duct leakage, cooling equipment charge, and combustion appliance backdrafting with spillage. We expect that a trained crew will need from 4 to 6 personhours to commission a house, excluding any corrective adjustments and retrofits to the building and its systems. Most tests can be performed using an equipment package priced toward the lower end of the $\$ 6,000$ and $\$ 15,000$ equipment cost spectrum.

Potential Benefits. Simulations by Matson et al. (2002) of a set of hypothetical California houses indicate that the potential benefits from applying whole-house commissioning range from almost nothing in some well-engineered advanced new houses to substantial savings in poorly-performing existing houses: as much as 70 to $80 \%$ savings in HVAC electricity and natural gas consumption, HVAC operating costs, and 
total HVAC fuel-related carbon emissions. Potential savings for typical houses are about $1 / 3$ to $1 / 2$ of the savings in the poorly performing houses.

In addition to these quantitative benefits, there is a broad spectrum of potential long-term qualitative benefits. Significant ones include more satisfied consumers, reduced callbacks and warranty costs for builders, improved abilities to enforce existing and future energy codes, greater confidence in predicting energy use, and greater assurance that energy efficiency reductions will actually occur.

Guidelines. We have produced a set of guidelines for developing a residential commissioning program that could be integrated with other building industry processes to achieve the benefits that we have identified. These guidelines provide specific recommendations about: how to structure the commissioning process, which diagnostics to use for each house component, and how to use them in a whole-house approach to commission new and existing houses. The guidelines are attached as Appendix I.

Outreach. Apart from our information dissemination to Project Advisory Committee (PAC) members and California Energy Commission staff over the course of the project, there have been substantial other activities to transfer project results to the building industry. Examples include: workshops to discuss the guidelines; presenting papers on residential commissioning topics; participating in related industry focus groups and standards development activities; and preparing a residential commissioning website (http://commissioning.lbl.gov/).

Conclusions. The primary outcomes from this project are the development of programmatic guidelines for residential commissioning and the analytical confirmation that there are significant potential benefits associated with commissioning California houses, particularly existing ones. In addition, we have made substantial advances in understanding the accuracy and usability of diagnostics for commissioning houses. In some cases, we have been able to work with equipment manufacturers to improve these aspects of their diagnostic tools. These outcomes provide a solid foundation on which to build a residential commissioning program in California. A concerted effort will be necessary to integrate such a program with existing building industry efforts and to demonstrate its use in the field, such that residential commissioning can ultimately improve house performance for consumers and the energy cost/value of electricity for the state.

Recommendations. Based on the project findings, our recommendations for further work are:

- Develop a practical diagnostic to evaluate the in-situ thermal conductance of envelope assemblies.

- Develop formal standards for assessing water damage and measuring the moisture content in building assemblies; for calibrating and using airflow diagnostic tools, such as those used to measure distribution system airflows at grilles or through the air-handler; and for superheat and subcooling tests of cooling equipment.

- Develop a method of assessing refrigerant charge in cool weather, and examine the utility of temporarily elevating indoor enthalpy in hot, dry weather, in order to extend the periods when the superheat method can be used to test cooling equipment.

- Assess the impact of wind effects, as well as envelope and duct leakage, on the accuracy and repeatability of duct leakage tests, including the new DeltaQ test. Related work is currently underway with funding from the U.S. Department of Energy and would benefit from co-funding by the California Energy Commission. 
- Further assess the accuracy and repeatability of methods that determine the potential for backdrafting and combustion gas spillage.

- Develop a simple and reliable test for furnace heat exchanger leakage. The norm for the current tracer gas test is poorly defined and may be unreliable for defining acceptable leakage levels of combustion gas products.

- Carry out field demonstrations to confirm that the benefits we have identified can be achieved by applying whole-house commissioning to new and existing California houses.

- Develop and implement a whole-house residential commissioning program for California that is integrated as much as possible with existing building industry processes.

- Continue collaborative work with the U.S. Department of Energy, industry organizations, and the private sector to transfer information to the building industry.

Benefits to California. We have identified several benefits that result directly from this study or that will accrue over time as necessary information and infrastructure develops further:

Electricity and gas consumption benefits. A principal benefit from commissioning houses is significant energy savings. For poorly-performing existing houses, we estimate that commissioning could achieve as much as 70 to $80 \%$ savings in HVAC electricity and natural gas consumption and HVAC operating costs; potential savings for typical houses are much less, but still significant (about $1 / 3$ to $1 / 2$ of these savings). It is difficult to extrapolate these savings over the entire state at this time, because the distribution of houses with problems is poorly defined. As the practice of commissioning grows, this activity will provide a statistical dataset that can be used to make such estimates.

Carbon emission benefits. Another significant benefit from residential commissioning is carbon emission savings resulting from reduced electricity and gas consumption. For poorly-performing existing houses, we estimate that commissioning could achieve as much as $80 \%$ reductions in total HVAC fuel-related carbon emissions. Like the energy savings described above, the potential carbon emission savings for typical houses are about $1 / 3$ to $1 / 2$ of the savings for the poorly-performing houses.

Durability, maintenance, and material replacement benefits. Through commissioning, we expect that the durability of building components will be improved and maintenance and material replacement activities and costs will be reduced. This translates into reduced use of natural resources and correspondingly, a lower embodied energy (the amount of energy required for manufacturing, construction, and deconstruction and waste reduction and decay) over the lifetime of the house.

Thermal comfort and indoor air quality benefits. Commissioning to reduce uncontrolled air infiltration, to provide appropriate ventilation capacity, and to achieve more consistent surface temperatures through better-installed insulation can help reduce moisture and comfort problems. It will also help ensure that the HVAC systems can deliver the expected amount of space conditioning capacity.

Economic benefits. The economy is a direct beneficiary of the building improvements implemented in residential commissioning. Commissioning contributes to reduced energy costs, which frees up increased funds for other purposes; expanded business opportunities; the development of new industries; increased jobs for California residents; and increased tax revenues. 


\section{INTRODUCTION}

\subsection{Background}

California has one of the most advanced energy codes in the United States: Title 24 (California Energy Commission 1998). In spite of this, California houses still do not perform optimally, or even as many forecasts predict based on expectations of this code.

For example, Walker et al. (1998) found large variations in duct leakage of California houses, even between side-by-side houses with the same system design and installation crew. This has resulted in as much as factor of two variations in thermal distribution system efficiency for these houses. This and other studies (e.g., Jump et al. 1996) indicate that duct leakage testing and sealing can readily improve thermal distribution system efficiency and achieve a 25 to $30 \%$ reduction in installed cooling capacity and energy consumption.

As another example, consider that for at least 20 years the building industry has recognized the substantial impact of envelope airtightness on thermal loads, energy use, comfort, and indoor air quality. However, Walker et al. (1998) found 50\% variances in airtightness for California houses with the same design and construction crews, within the same subdivision.

A substantial reason for these problems is that few houses are now built or retrofitted using formal design procedures, most are field assembled from a large number of components, and there is no consistent process to identify related energy and non-energy problems or to correct them. Solving the problems requires field performance evaluations of new and existing houses using appropriate and agreed upon procedures. Many procedural elements already exist in a fragmented environment; some are ready now to integrate into a new process called residential commissioning (Wray et al. 2000). California's Title 24 energy code already provides some commissioning elements for evaluating the energy performance of new houses.

The overall goal of this project is to develop and document residential commissioning procedures and to demonstrate the value that applying these procedures could provide in both new and existing houses. We expect that this new information will lay the groundwork for a residential commissioning industry in California, which ultimately will increase the use of commissioning for California houses. In turn, we expect that the application of commissioning by people such as home energy raters, home inspectors, auditors, and weatherization contractors will improve or even optimize residential energy and indoor environmental performance, and will contribute to improving the energy cost/value of electricity for the state.

This project contributes to the PIER program objective of improving the energy cost and value of California's electricity in three ways. One is by evaluating methods and developing guidelines for reliably identifying and correcting performance problems associated with deficiencies in interacting house components and systems. A second is to eliminate a barrier to performing residential commissioning by providing information on the value of performing residential commissioning services in California houses. A third is by conducting outreach activities to make building industry stakeholders aware of this newly developed information. We expect that the knowledge gained from this research will ultimately be used to develop a robust residential commissioning program in California that is integrated with existing building industry efforts and that can then be used to confirm the value of commissioning through field demonstrations. 


\subsection{Project Objectives}

The specific technical objectives for this project are to:

1. Define, assess, and articulate metrics and diagnostics ("yardsticks" and measurement techniques) for residential commissioning, with a focus on delineating them based upon their accuracy and usability in the field.

2. Provide information on the potential benefits of commissioning California houses using a whole-house approach, which recognizes that a house is a system of interacting components.

3. Develop programmatic guidelines for commissioning new and existing California houses.

4. Conduct outreach efforts to transfer project results, such as the residential commissioning guidelines, to industry stakeholders who will use the results of the work in program, code, standard, and technology development.

\subsection{Report Organization}

This report presents our findings and recommendations that have resulted from investigating the practicality and value of commissioning residential buildings. Most of the work focuses on commissioning the energy performance of energy-related building components and systems rather than on indoor air quality and thermal comfort impacts, because energy-related diagnostics are currently much better developed for use in a process like residential commissioning.

In Section 2 Project Approach, we discuss the tasks that we undertook and our approach to the research to accomplish our objectives. In particular, we discuss changes to the field-testing procedures that we undertook and the need for diagnostic tool evaluations in those tests.

In Section 3 Project Outcomes, we present the key results from our investigations.

In Section 4 Conclusions and Recommendations, we present what we learned from the research and what we recommend for future activities.

Following the Glossary and References, there are three technical Appendices:

"Appendix I. Guidelines for Residential Commissioning" provides a guide for industry stakeholders who are interested in developing whole-house residential commissioning programs. These stakeholders include managers of government and utility energyefficiency programs, representatives of home performance contractor and home energy rater organizations, representatives of builder associations, executives of home inspection and quality home companies, and code official representatives.

Until a program exists that requires specific aspects of commissioning, it is premature to include step-by step procedures in a comprehensive commissioning guide. As a result, this guide is not a test procedure, design, construction, installation specification, home inspection, operation and maintenance, or retrofit/repair manual. Many documents targeted specifically to individual areas in this list already exist, and this guide points to such existing references where appropriate. Even after the building industry develops and implements a commissioning program, it is probably better to leave such details to these documents, which experts in each specific area can update over time within focused consensus processes that are coordinated with each other. 
"Appendix II. Example Audit and Diagnostic Process" provides a flow chart to illustrate a possible sequence for carrying out tasks in the initial audit and diagnostic phase of the residential commissioning process. Supporting text in a table summarizes the commissioning tasks by describing what each task does, what test protocols could be used, what equipment is needed, how long each task takes, and the potential energy savings associated with carrying out each task.

"Appendix III. Example Commissioning Report" provides a sample report that could be used to document the audit and diagnostic findings from commissioning a house. The data included in the report are for an actual house in Concord, California, which has been retrofitted recently as part of a DOE demonstration of residential commissioning.

\section{PROJECT APPROACH}

To achieve the project goals and objectives, the original project plan divided technical tasks into three groups that addressed multiple project objectives in some cases:

- Develop a commissioning process, metrics, and diagnostic approaches to evaluate and improve the energy and non-energy performance of new and existing California houses. A review of literature related to building commissioning was expected to provide information that could be used to select appropriate metrics and diagnostics. Where necessary, we anticipated that some adaptation or minor development of existing diagnostics might be needed to make them more practical for use in residential commissioning.

- Carry out a potentials study to assess the utility and accuracy of the selected metrics and diagnostics in field applications of the commissioning process and quantify the energy savings associated with using the recommended commissioning process. The first part of this effort was to involve field tests of the diagnostics to confirm that the commissioning process we recommended was practical and robust. The second part was to involve computer simulations that demonstrate the potential benefits of carrying out commissioning on various types of hypothetical houses located in various California climates.

- Prepare residential commissioning guidelines to describe the process and the metrics and diagnostics, and to provide guidance on when and how to use the diagnostics. In addition, to obtain feedback and fine-tune the guidelines, conduct outreach activities through workshops for the residential building industry in California.

As in any research effort, the results along the way also shaped the work. Based on input from the PAC and the PIER Buildings Program team, the project evolved. Changes included:

- Our focus shifted away from non-energy-related indoor air quality (IAQ) and thermal comfort diagnostics. At the conclusion of our literature review, it was clear that many well-developed diagnostics are already available to determine non-energy-related metrics. However, they tend to be complex, time consuming, and require expensive equipment or analyses and well-trained diagnosticians. Without selecting an appropriate diagnostic to target specific concerns or dissatisfaction of occupants, the cost of attempting to address the apparent myriad of possible comfort and IAQ problems is prohibitive. As a result, most diagnostics to assess IAQ and comfort tend to be impractical for residential commissioning at this time. 
- Cofunding by the U.S. Department of Energy, as well as related efforts in projects for the California Institute for Energy Efficiency (CIEE) and Pacific Gas and Electric, allowed us to carry out more thorough tests of energy-related diagnostics in the laboratory and field, and to develop a better understanding of the accuracy and usability of these diagnostics (Wray et al. 2002a). The outcome was that many of these diagnostics (e.g., flow hoods) still needed significant development to be useful within a residential commissioning process.

Consequently, we focused our field efforts within this project on further understanding and improving the accuracy and usability of the diagnostics rather than carrying out case studies of houses where the entire commissioning process might have been applied. We also carried out more information dissemination than expected so that our findings would be documented in the archival literature.

Appendix I, along with Wray et al. 2000, Matson et al. 2002, and Wray et al. 2002a, describe our research efforts in detail.

\section{PROJECT OUTCOMES}

This section presents the key results from our investigations, in the same order as the three objectives.

Objective \#1: Define, assess, and articulate metrics and diagnostics ("yardsticks" and measurement techniques) for residential commissioning, with a focus on delineating them based upon their accuracy and usability in the field.

Metrics \& Diagnostics. We identified and described metrics for use in residential commissioning, along with a consolidated set of 24 practical diagnostics that the building industry can use now to evaluate them (Wray et al. 2002a). This set focuses on existing diagnostics in areas of particular concern where there are significant component and system interactions: envelope insulation quality, windows, airtightness, envelope moisture, HVAC fan and duct system airflows, duct leakage, cooling equipment charge, and combustion appliance backdrafting with spillage.

This assessment of metrics and diagnostics is based upon our recent literature review and annotated bibliography for this project (Wray et al. 2000), which facilitates access to 469 documents related to residential commissioning that have been published over the past 20 years. It is also based upon recent laboratory work and field studies in more than 100 houses by LBNL staff and others to evaluate the accuracy and usability of diagnostics. These studies concentrated on evaluating diagnostics in the following four areas: the DeltaQ duct leakage test, air-handler airflow tests, supply and return grille airflow tests, and refrigerant charge tests

Individual equipment costs for the 24 practical diagnostics range from a few hundred dollars to many thousands of dollars; total equipment costs are expected to be between $\$ 6,000$ and $\$ 15,000$. The higher costs are associated with infrared thermography and state-of-the-art automated diagnostic systems. Most tests can be performed in one hour or less, using equipment priced toward the lower end of the cost spectrum. We expect that a trained crew will need from 4 to 6 person-hours to commission a house, excluding any corrective adjustments and retrofits to the building and its systems.

The equipment prices listed here are based on data provided by manufacturers and distributors as of April 2001. These prices may change in the future as residential 
commissioning activities increase. Note that the total price for a diagnostic toolkit to carry out all tests is not the sum of each cost listed. In many cases, the equipment from one diagnostic can be used for other diagnostics, often with only slight modifications. An example is the envelope airtightness test and DeltaQ duct leakage test, where both tests use the same equipment (the DeltaQ test uses a blower door and a pressure measurement device to simultaneously determine envelope airtightness and duct leakage). Another example is using a fan-assisted flow meter to determine air-handler airflow and a fanassisted flow hood to determine duct airflows. Both devices use a fan-assisted flow meter; the latter also has a flow capture hood.

Objective \#2: Provide information on the potential benefits of commissioning California houses using a whole-house approach, which recognizes that a house is a system of interacting components.

Potential Benefits. Based on simulations by Matson et al. (2002) of a set of hypothetical California houses, commissioning can result in better performing systems and houses. In turn, this will result in more efficient use of energy, carbon emission reductions, and improved occupant comfort. In particular, on the spot adjustments to houses ("tuning and tweaking") can save a significant amount of HVAC-related energy (15 to 30\% in existing houses, 10 to $20 \%$ in new conventional houses, and up to $8 \%$ in advanced energy efficiency houses). The process that we considered includes corrective measures that could be implemented together during construction or during a single site visit (e.g., air tightening, duct sealing, and refrigerant and air handler airflow corrections in a new or existing house). Taking advantage of additional, more complex opportunities (e.g., installing new windows in an existing house, replacing the heating and air conditioning system in a new or existing house) can result in additional HVAC-related energy savings (60 to $75 \%$ in existing houses, and 50 to $60 \%$ in new conventional houses).

Consequently, the potential benefits from applying whole-house commissioning range from almost nothing in some well-engineered advanced new houses to substantial savings in poorly-performing existing houses: as much as $80 \%$ savings in HVAC electricity consumption, 70\% savings in HVAC natural gas consumption, 70\% HVAC operating cost savings, and $80 \%$ reductions in total HVAC fuel-related carbon emissions. For typical houses, the potential savings are only about $1 / 3$ to $1 / 2$ of the savings in the poorly performing houses, because the typical houses have fewer opportunities for change. Field demonstrations are needed to confirm that these benefits can be achieved by applying whole-house commissioning to new and existing California houses.

The commissioning-related system and house performance improvements and energy savings translate to additional benefits throughout California and beyond. By applying commissioning principles to their work, the building community (builders and contractors) benefit from reduced callbacks and lower warranty costs. Home energy raters and home inspectors will have access to an expanded market sector. As the commissioning process rectifies construction defects and code problems, building code officials benefit from better compliance with codes. The utilities benefit from reduced peak demand, which can translate into lower energy acquisition costs. As houses perform closer to expectations, governmental bodies (e.g., the California Energy Commission and the Air Resources Board) benefit from greater assurance that actual energy consumption and carbon emissions are closer to the levels mandated in codes and standards, resulting in better achievement of state energy conservation and environmental goals. California residents' quality of life is improved through better indoor environmental comfort and 
lower energy bills. Lower energy bills free up money for residents to spend on other needs or goals, such as additional education and health and welfare. With an expansion of existing industries and the development of new commissioning-related industries, related jobs and tax revenues will increase, further increasing the quality of life for California.

Objective \#3: Develop programmatic guidelines for commissioning new and existing California houses.

Guidelines. We have produced a set of guidelines (Appendix I) for developing a residential commissioning program that can be integrated with other building industry processes to achieve the benefits that we have identified. The guidelines contain three sections. The first section provides an overview of why commissioning is needed and why looking at the house as a system rather than as isolated components is an important element of commissioning. It then describes the characteristic elements of commissioning and how we envision the process would work. This section concludes with a discussion of cost barriers to commissioning and who might do the work.

The second section of the guide discusses the application of commissioning to new and existing houses. For each of these house types, we provide an example to illustrate whole-house commissioning and its benefits. In each example, we first describe the precommissioning state of the house, as could be defined by diagnostic testing. We then describe simple tuning and tweaking improvements to the houses, and then opportunities for further but more complex improvements, with the intent that all the changes could be verified using diagnostic tests.

We have subdivided the third section of the guide into four subsections, each of which discusses the commissioning of a specific house component: the building envelope, air distribution systems, cooling equipment, and combustion appliances. For each component, the relevant subsection discusses what is being commissioned, why testing is needed, applicable metrics, what tests are recommended, and how to choose a particular test from the recommended set. For each test, it also describes equipment needs, their relative cost, and their accuracy (as appropriate and where information is available). The subsection for each component ends with a list of available procedural references that the reader can consult for further information.

In terms of who should do the commissioning, it is important to note that there are several active new construction and home retrofit energy-efficiency programs within California, but few people are currently qualified to commission houses on a wholehouse basis (Knight and Thomas 2000, Wirtshafter Associates 2000). Perhaps the best qualified at this time are home energy raters and building performance contractors, who typically have more building science training and a better understanding of the house-asa-system concept than any other group. If these independent parties are used to commission houses, we envision that builders and perhaps subcontractors may still carry out some commissioning themselves throughout the construction process in preparation for the independent commissioning. However, careful integration of the building team and independent party commissioning with the construction process is important to avoid scheduling conflicts and unnecessary cost. Involving independent parties in the construction process has been problematic in the past (Pacific Consulting Services 2000). For whole-house residential commissioning programs to succeed, training, certification, accreditation, and commissioning management processes need to be developed and 
widely used. We anticipate that involving state agencies and building industry associations in the development of such processes is key.

Objective \#4: Conduct outreach efforts to transfer project results, such as the residential commissioning guidelines, to industry stakeholders who will use the results of the work in program, code, standard, and technology development.

Outreach. Apart from our information dissemination to Project Advisory Committee (PAC) members and California Energy Commission staff at four technical review meetings over the course of the project, there have been substantial other activities to transfer project results to the building industry. Examples of these activities include:

- hosting a guidelines scoping workshop entitled "Defragmenting Your House: Guides for Residential Commissioning" at the ACEEE 2000 meeting (the objective of this workshop was to summarize work to date and to obtain industry input on a format for the commissioning guide, its technical level, and how commissioning can be integrated into existing building industry processes);

- presenting the draft guidelines to invited building industry stakeholders at a workshop to obtain feedback;

- presenting various papers on the concept of residential commissioning and on the accuracy and usability of diagnostic equipment (Wray and Sherman 2001; Walker et al. 2001; Siegel and Wray 2002; Wray et al. 2002b);

- participating in industry focus groups and standards development related to building commissioning (For example, the "Leadership in Energy and Environmental Design - Residential" (LEED-R) Green Building Rating System is being developed by the United States Green Building Council (USGBC). We have been participating in the development of this rating system to incorporate residential commissioning. In particular, we have submitted comments regarding suggested changes to elements of the draft LEED-R document related to performance verification. The revised draft document has incorporated most of our comments.);

- discussions with diagnostic equipment manufacturers about changes needed to improve accuracy and usability (some manufacturers have expressed a continued interest about participating in laboratory and field tests to further evaluate measurement techniques, with a view toward developing improved technologies for residential commissioning); and

- preparing a residential commissioning website hosted by LBNL (http://commissioning.lbl.gov/). 


\section{CONCLUSIONS AND RECOMMENDATIONS}

\subsection{Conclusions}

The primary outcomes from this project are the development of programmatic guidelines for residential commissioning and the analytical confirmation that there are significant potential benefits associated with commissioning California houses, particularly existing ones. In addition, we have made substantial advances in understanding the accuracy and usability of diagnostics for commissioning houses. In some cases, we have been able to work with equipment manufacturers to improve these aspects of their diagnostic tools. These outcomes provide a solid foundation on which to build a residential commissioning program in California. A concerted effort will be necessary to integrate such a program with existing building industry efforts and to demonstrate its use in the field, such that residential commissioning can ultimately improve house performance for consumers and the energy cost/value of electricity for the state.

\subsection{Commercialization Potential}

The output of this project is new knowledge about how to evaluate the energy performance of houses, about the usability and accuracy of specific diagnostic technologies both old and new, and about the value of applying commissioning to California houses. Apart from proposing a commissioning process, this project does not develop a particular technology, but it does provide a blueprint for the nascent commissioning industry that will improve end-use energy efficiency and will further develop improved diagnostic technologies.

In the near term, we expect that the results of this project will lower the implementation barriers for technologies and services already known. Our tools and evaluations give users confidence in the performance (or non-performance) of many housing components. In the medium term, we expect to see new kinds of services develop that would use the results of our project to facilitate a new kind of rating, auditing, or commissioning. In the long term, we expect to see innovative replacements for the lower performing equipment installed in the housing stock and more care taken in correct installation (and operation and maintenance) of envelope and HVAC systems. Industrialized construction, smart envelopes, and whole-building control may all factor into these designs.

We expect that commissioning could be integrated as part of California Title 24, which would potentially allow the energy budget of the integrated design to be increased, while at the same time lowering the actual energy usage of the building. Integrating commissioning with Title 24 will create a new value-added service that also has the indirect effect of lowering the value of products and installations that do not easily perform as expected and raising the value of those that meet or exceed their specifications. Commissioning would allow the market to more properly value different technologies and should have the effect of increasing the market presence/share of the good ones. Steps in this direction have already begun through the incorporation of additional performance testing requirements in the recent $\mathrm{AB} 970$ revisions to Title 24 .

This project has benefited from over $\$ 400 \mathrm{k}$ of support from the Building Technologies office at the U.S. Department of Energy. DOE plans to continue supporting work in this area, including continued efforts to develop residential commissioning diagnostics, and best practice guidelines for retrofitting houses to address performance problems. 


\subsection{Recommendations}

Based on our findings, our recommendations for further work are as follows, arranged in the same order as the three objectives:

Objective \#1: Define, assess, and articulate metrics and diagnostics ("yardsticks" and measurement techniques) for residential commissioning, with a focus on delineating them based upon their accuracy and usability in the field.

Recommendation \#1: Develop a practical diagnostic to evaluate the in-situ thermal conductance of envelope assemblies.

Recommendation \#2: Develop formal standards for: assessing water damage and measuring the moisture content in building assemblies; calibrating and using airflow diagnostic tools, such as those used to measure distribution system airflows at grilles or through the air-handler; and superheat and subcooling tests of cooling equipment.

Recommendation \#3: Develop a method of assessing refrigerant charge in cool weather, and examine the utility of temporarily elevating indoor enthalpy in hot, dry weather, in order to extend the periods when the superheat method can be used to test cooling equipment.

Recommendation \#4: Assess the impact of wind effects, as well as envelope and duct leakage, on the accuracy and repeatability of duct leakage tests, including the new DeltaQ test. This work is currently underway with funding from the U.S. Department of Energy and would benefit from co-funding by the California Energy Commission.

Recommendation \#5: Further assess the accuracy and repeatability of methods that determine the potential for backdrafting and combustion gas spillage.

Recommendation \#6: Develop a simple and reliable test for furnace heat exchanger leakage. The norm for the current tracer gas test is poorly defined and may be unreliable for defining acceptable leakage levels of combustion gas products.

Objective \#2: Provide information on the potential benefits of commissioning California houses using a whole-house approach, which recognizes that a house is a system of interacting components.

Recommendation \#7: Carry out field demonstrations to confirm that the benefits we have identified can be achieved by applying whole-house commissioning to new and existing California houses.

Objective \#3: Develop programmatic guidelines for commissioning new and existing California houses.

Recommendation \#8: Develop and implement a whole-house residential commissioning program for California that is integrated as much as possible with existing building industry processes.

Objective \#4: Conduct outreach efforts to transfer project results, such as the residential commissioning guidelines, to industry stakeholders who will use the results of the work in program, code, standard, and technology development.

Recommendation \#9: Continue collaborative work with the U.S. Department of Energy, industry organizations, and the private sector to transfer information to the building industry. 


\subsection{Benefits to California}

We have identified the following benefits that result directly from this study or that will accrue over time as necessary information and infrastructure develops further:

Electricity and gas consumption benefits. One of the principal benefits from commissioning houses is significant energy savings. For poorly-performing existing houses, we estimate that commissioning could achieve as much as $80 \%$ savings in HVAC electricity consumption and 70\% savings in HVAC natural gas consumption $(70 \%$ HVAC operating cost savings); potential savings for typical houses are much less, but still significant (about $1 / 3$ to $1 / 2$ of these savings). It is difficult to extrapolate these savings over the entire state at this time, because the distribution of houses with problems remains poorly defined. As the practice of commissioning grows in California, data from such activities will provide a statistical profile that can be used to make such estimates.

Carbon emission benefits. Another significant benefit from residential commissioning is carbon emission savings resulting from reduced electricity and gas consumption. For poorly-performing existing houses, we estimate that commissioning could achieve as much as $80 \%$ reductions in total HVAC fuel-related carbon emissions. Like the energy saving described above, the potential carbon emission savings for typical houses are about $1 / 3$ to $1 / 2$ of these savings.

Durability, maintenance, material replacement, and house resale value benefits. Through commissioning, we expect that building envelope and equipment durability will be improved and maintenance and material replacement activities and costs will be reduced. In terms of the environment, this translates into a lower use of natural resources and correspondingly, a lower embodied energy (the amount of energy required for manufacturing, construction, and deconstruction and waste reduction and decay) over the lifetime of the house. Increased energy savings can increase house resale values (Nevin and Watson 1998).

Thermal comfort and indoor air quality benefits. Commissioning to reduce uncontrolled air infiltration, to provide appropriate ventilation capacity, and to achieve more consistent surface temperatures through better-installed insulation can help reduce moisture and comfort problems. It will also help ensure that the HVAC systems can deliver the expected amount of space conditioning capacity.

Economic benefits. The economy, within and beyond the State of California, is a direct benefactor of the building improvements implemented in residential commissioning. Commissioning contributes to reduced energy costs, which frees up increased funds for other purposes; expanded business opportunities; the development of new industries; increased jobs for California residents; and increased tax revenues. 


\section{GLOSSARY}

ACEEE American Council for an Energy-Efficient Economy

ASHRAE American Society of Heating, Refrigerating, and Air-Conditioning Engineers

CIEE California Institute for Energy Efficiency

DOE U.S. Department of Energy

HVAC Heating, ventilating and air conditioning

IAQ Indoor air quality

LBNL Lawrence Berkeley National Laboratory

LEED-R "Leadership in Energy and Environmental Design - Residential” - Green Building Rating System

PAC Project Advisory Committee

PIER Public Interest Energy Research

RD\&D Research, Development, and Demonstration

UC University of California 


\section{ACKNOWLEDGEMENTS}

The authors wish to acknowledge the significant contributions of Energy Performance of Buildings Group staff and students who were involved in analytical and experimental work that directly supported the outcomes of this project: Bass Abushakra, Darryl Dickerhoff, Jennifer McWilliams, Nance Matson, Jeff Siegel, Duo Wang, and Jacob Wempen. We also wish to acknowledge the technical assistance and field support of the designers and manufacturers of the equipment that we evaluated in this project.

Our Project Advisory Committee (PAC) members contributed at several critical junctures, and we would like to acknowledge their support and input. The PAC members are:

Ed Becker, Southern California Gas Company;

Karl Brown, California Institute for Energy Efficiency;

Rick Chitwood, Chitwood Energy Management;

Lance Elberling, Pacific Gas and Electric Company;

David Goldstein, Natural Resources Defense Council;

Raymond Hall, H\&L Energy Savers;

Rob Hammon, ConSol Inc.;

Marshall Hunt, Pacific Gas and Electric Company;

Scott Johnson, Action Now!;

Joe Lstiburek, Building Science Corporation;

Bill Pennington, California Energy Commission;

John Proctor, Proctor Engineering Group;

Robert Raymer, California Building Industry Association;

Robert Scott, California Home Energy Efficiency Rating System;

Bruce Wilcox, Berkeley Solar Group; and

Rick Wylie, Beutler Heating \& Air Conditioning Inc.

This work was also supported by the Assistant Secretary for Energy Efficiency and Renewable Energy, Building Technologies Program, of the US Department of Energy under Contract No. DE-AC03-76SF00098 through Esher Kweller, Program Manager, U.S. Department of Energy, who also provided important technical and programmatic suggestions.

Lastly, we would like to acknowledge the support and contributions of the PIER Contract Managers, Chris Scruton, Martha Brook, Dale Trenschel, and Ann Peterson, as well as the rest of the Buildings Program team under the leadership of Nancy Jenkins. 


\section{REFERENCES}

California Energy Commission. 1998. "Low-Rise Residential Alternative Calculation Method Approval Manual for 1998 Energy Efficiency Standards for Low-Rise Residential Buildings". Sacramento, CA: California Energy Commission. http://www.energy.ca.gov/title24/residential acm/index.html.

Jump, D.A., I.S. Walker, and M.P. Modera. 1996. "Field Measurements of Energy Efficiency and Duct Retrofit Effectiveness in Residential Forced Air Distribution Systems". Proceedings, 1996 ACEEE Summer Study on Energy Efficient Buildings. Washington, D.C.: American Council for an Energy-Efficient Economy.

Knight, R.L. and G. Thomas. 2000. "Whole-House Contractor Team Accreditation:

Development of a Feasible Model and Implementation Process". Report of Performance Systems Development, Inc. and Bevilacqua-Knight, Inc. to California Energy Commission. P400-00013CR. July.

Matson, N.E., C.P. Wray, I.S. Walker, and M.H. Sherman. 2002. "Potential Benefits of Commissioning California Homes". Lawrence Berkeley National Laboratory report LBNL48258. http://epb1.lbl.gov/EPB/Publications/lbnl-48258.pdf.

Nevin, R. and G. Watson. 1998. "Evidence of Rational Market Valuations for Home Energy Efficiency.” The Appraisal Journal, October 1998, The Appraisal Institute, Chicago, Illinois.

Pacific Consulting Services. 2000. "Market Assessment \& Evaluation Study in Support of Codes and Standards". Final Report of Pacific Consulting Services, Davis Energy Group, and Eley Associates to Pacific Gas \& Electric. PG\&E Study ID number 411. August 31.

Siegel, J.A. and C.P. Wray. 2002. "An Evaluation of Superheat-Based Refrigerant Charge Diagnostics for Residential Cooling Systems". Lawrence Berkeley National Laboratory report LBNL-47476. http://epb1.lbl.gov/EPB/Publications/lbnl-47476.pdf. Also in ASHRAE Transactions, Vol. 108, Part 2. Atlanta, GA: American Society of Heating Refrigerating Engineers, Inc.

Walker, I., M. Sherman, M. Modera, and J. Siegel. 1998. "Leakage Diagnostics, Sealant Longevity, Sizing and Technology Transfer in Residential Thermal Distribution Systems". Lawrence Berkeley National Laboratory report LBNL-41118. http://epb1.lbl.gov/EPB/Publications/lbl-41118.pdf.

Walker, I.S., M.H. Sherman, J. Wempen, D. Wang, J.A. McWilliams, and D.J. Dickerhoff. 2001. "Development of a New Duct Leakage Test: Delta Q". Lawrence Berkeley National Laboratory report LBNL-47308. http://epb1.lbl.gov/EPB/Publications/lbnl-47308.pdf.

Wirtshafter Associates. 2000. "Residential Contractor Program Evaluation, Phase II Final Report”. Quakertown, PA: Volume 1, Summary Report. July 10.

Wray, C.P., M.A. Piette, M.H. Sherman, R.M. Levinson, N.E. Matson, D.A. Driscoll, J.A. McWilliams, T.T. Xu, and W.W. Delp. 2000. "Residential Commissioning: A Review of Related Literature". Lawrence Berkeley National Laboratory report LBNL-44535. http://epb1.lbl.gov/EPB/Publications/lbnl-44535.pdf.

Wray, C.P. and M.H. Sherman. 2001. "Residential Commissioning to Assess Envelope and HVAC System Performance”. Lawrence Berkeley National Laboratory report LBNL-47412. http://epb1.lbl.gov/EPB/Publications/lbnl-47412.pdf. Also in Proceedings - Performance of 
Exterior Envelopes of Whole Buildings VIII Conference, Clearwater Beach, FL, December. Atlanta, GA: American Society of Heating Refrigerating Engineers, Inc.

Wray, C.P., I.S. Walker, J.A. Siegel, and M.H. Sherman. 2002a. "Practical Diagnostics for Evaluating Residential Commissioning Metrics" Lawrence Berkeley National Laboratory report LBNL-45959. http://epb1.lbl.gov/EPB/Publications/lbnl-45959.pdf.

Wray, C.P., I.S. Walker, and M.H. Sherman. 2002b. "Accuracy of Flow Hoods in Residential Applications". Lawrence Berkeley National Laboratory report LBNL-49697. http://epb1.lbl.gov/EPB/Publications/lbnl-49697.pdf. Also in Proceedings, 2002 ACEEE Summer Study on Energy Efficient Buildings. Washington, D.C.: American Council for an Energy-Efficient Economy. 


\section{APPENDIX I. GUIDELINES FOR RESIDENTIAL COMMISSIONING}

Craig Wray, Max Sherman, and Iain Walker

Lawrence Berkeley National Laboratory

Berkeley, California 


\section{PREFACE}

Currently, houses do not perform optimally or even as many codes and forecasts predict, largely because they are field assembled and there is no consistent process to identify problems or to correct them. Residential commissioning is a solution to this problem.

This guide is the culmination of a 30-month project that began in September 1999. The ultimate objective of the project is to increase the number of houses that undergo commissioning, which will improve the quality, comfort, and safety of homes for California citizens. The project goal is to lay the groundwork for a residential commissioning industry in California focused on end-use energy and non-energy issues. As such, we intend this guide to be a beginning and not an end. Our intent is that the guide will lead to the programmatic integration of commissioning with other building industry processes, which in turn will provide more value to a single site visit for people such as home energy auditors and raters, home inspectors, and building performance contractors.

Project work to support the development of this guide includes:

- a literature review and annotated bibliography, which facilitates access to 469 documents related to residential commissioning published over the past 20 years (Wray et al. 2000),

- an analysis of the potential benefits one can realistically expect from commissioning new and existing California houses (Matson et al. 2002), and

- an assessment of 107 diagnostic tools for evaluating residential commissioning metrics (Wray et al. 2002).

In this guide, we describe the issues that non-experts should consider in developing a commissioning program to achieve the benefits we have identified. We do this by providing specific recommendations about: how to structure the commissioning process, which diagnostics to use, and how to use them to commission new and existing houses. Using examples, we also demonstrate the potential benefits of applying the recommended whole-house commissioning approach to such houses. 


\section{INTRODUCTION}

\section{Purpose}

The purpose of this guide is to describe the residential commissioning process, to identify which components in a house need commissioning, to recommend how to commission them, and to provide examples that demonstrate the benefits of whole-house commissioning.

\section{Scope}

We have developed this document as a guide for industry stakeholders who are interested in developing whole-house residential commissioning programs. These stakeholders include managers of government and utility energy-efficiency programs, representatives of home performance contractor and home energy rater organizations, representatives of builder associations, executives of home inspection and quality home companies, and code official representatives.

Until a program exists that requires specific aspects of commissioning, it is premature to include step-by step procedures in a comprehensive commissioning guide. As a result, this guide is not a test procedure, design, construction, installation specification, home inspection, operation and maintenance, or retrofit/repair manual. Many documents targeted specifically to individual areas in this list already exist, and this guide points to such existing references where appropriate. Even after the building industry develops and implements a commissioning program, it is probably better to leave such details to these documents, which experts in each specific area can update over time within focused consensus processes that are coordinated with each other.

\section{Structure}

This guide contains three sections. The first section provides an overview of why commissioning is needed and why looking at the house as a system rather than as isolated components is an important element of commissioning. It then describes the characteristic elements of commissioning and how we envision the process would work. This section concludes with a discussion of cost barriers to commissioning and who might do the work.

The second section of the guide discusses the application of commissioning to new and existing houses. For each of these house types, we provide an example to illustrate whole-house commissioning and its benefits. In each example, we first describe the pre-commissioning state of the house, as could be defined by diagnostic testing. We then describe simple tuning and tweaking improvements to the houses, and then opportunities for further but more complex improvements, with the intent that all the changes could be verified using diagnostic tests.

We have subdivided the third section of the guide into four subsections, each of which discusses the commissioning of a specific house component: the building envelope, air distribution systems, cooling equipment, and combustion appliances. For each component, the relevant subsection discusses what is being commissioned, why testing is needed, applicable metrics, what tests are recommended, and how to choose a particular test from the recommended set. For each test, it also describes equipment needs, their relative cost, and their accuracy (as appropriate and where information is available). The subsection for each component ends with a list of available procedural references that the reader can consult for further information. 


\section{BACKGROUND}

This section provides an overview of the emerging process of residential commissioning. We first describe the need for commissioning, particularly within a "house as a system" approach. Next, we describe what components need commissioning, the characteristic elements of the process, how we envision the process could be structured, the benefits of commissioning, and who could do it today. Note that this discussion is largely preliminary, because the practice of residential commissioning does not yet exist. A goal of this guide is to provide the basis for developing programs that address these residential commissioning issues.

\section{A Problem with a Solution}

California has one of the most advanced energy codes in the United States: Title 24 (CEC 1998). In spite of this, California houses still do not perform optimally, or even as many forecasts predict based on expectations of this code.

For example, Walker et al. (1998a) found large variations in duct leakage of California houses, even between side-by-side houses with the same system design and installation crew. This has resulted in as much as factor of two variations in thermal distribution system efficiency for these houses. This and other studies (e.g., Jump et al. 1996) indicate that duct leakage testing and sealing can readily improve thermal distribution system efficiency and achieve a 25 to $30 \%$ reduction in installed cooling capacity and energy consumption.

As another example, consider that for at least 20 years the building industry has recognized the substantial impact of envelope airtightness on thermal loads, energy use, comfort, and indoor air quality. However, Walker et al. (1998a) found 50\% variances in airtightness for California houses with the same design and construction crews, within the same subdivision.

A substantial reason for these problems is that few houses are now built or retrofitted using formal design procedures, most are field assembled from a large number of components, and there is no consistent process to identify related energy and non-energy problems or to correct them. Solving the problems requires field performance evaluations of new and existing houses using appropriate and agreed upon procedures. Many procedural elements already exist in a fragmented environment; some are ready now to integrate into a new process called residential commissioning (Wray et al. 2000). California's Title 24 energy code already provides some commissioning elements for evaluating the energy performance of new houses.

\section{Commissioning the House as a System}

The various building envelope and mechanical equipment components of a house interact to form a complex system that hopefully can produce an acceptable indoor environment for the occupants. Primary interactions are due to flows of air, heat, and moisture, all of which can affect occupant safety, health, or comfort, as well as building durability. Inevitably, when one component is installed incorrectly, or when it degrades over time or is modified, these flows change and there is a resulting impact on whole-house performance and the occupants.

Traditionally, new construction and retrofit markets construct or improve houses using a component-based approach with separate trades being responsible for individual pieces of the house. Because this approach does not deal with the house as a system, it cannot fully incorporate parallel issues of energy consumption, peak power, thermal comfort, pollutant control, or the benefits of multifunctional operations and associated synergies. 
As building technology, utility regulation, and homeowner needs have become more complicated to address historic problems of excessive energy consumption and discomfort, the traditional approach has become problematic. For example, consider that regulators and homeowners expected a substantial increase in whole-house energy efficiency due to the increases in equipment efficiency and envelope insulation levels of the 1980's. However, actual increases were less than expected, in part because the poor thermal efficiency of air distribution systems connecting these components was not accounted for when estimating the increases in wholehouse energy efficiency.

As a more specific example of the complicated interactions that occur when a house operates as a system, consider the impacts of a leaky supply and return duct system located in a hot unconditioned attic (attics can reach $150^{\circ} \mathrm{F}$ or more on sunny summer days). When the airhandler operates, hot air from the attic is drawn into the return leaks. This leakage raises the temperature of the return air and acts to reduce the effective capacity of the air-conditioning system. Conditioned air that leaks out of supply ducts is essentially lost to outside, because the attic is outside the thermal and air barrier of the building envelope. As a result, occupant comfort may be impaired because insufficient cooling is delivered to the rooms that the supply ducts serve (Walker et al. 1998b).

If there is an imbalance between the supply and return leaks, there are additional "indirect effects" of the duct leakage. The imbalance will result in pressurization (larger return leaks) or depressurization (larger supply leaks) of the house. These pressure changes in turn affect the infiltration rate of the house and thus the thermal and humidity load that the space-conditioning system must deal with to maintain comfort (Modera and Jansky 1992). In some cases, a larger capacity space conditioning system with a larger air-handler airflow will be required to accommodate the additional loads. When there is a central return grille, the increased return airflow can lead to excessive house depressurization if interior door undercuts are too small to allow supply air to flow easily from each room back to the return grille. If there is a naturallyaspirated combustion appliance in the house near the return, such as a fireplace, the resulting depressurization may contribute to backdrafting of the flue and indoor spillage of toxic combustion gas products. Operating an exhaust fan for local ventilation, such as a kitchen fan during cooking, can cause further depressurization.

Attempts to seal only the building envelope to reduce the infiltration and associated loads (without sealing the duct leaks) can exacerbate the combustion-related problems by increasing depressurization and reducing whole-house ventilation. They can also reduce the run time of the space conditioning system, thus reducing its efficiency due to increased cycling losses. During the cooling season, this can also result in poorer humidity control. It can also reduce mixing of air throughout the house, which in turn can lead to unacceptably warm rooms where there are significant solar loads.

In the case where the return leaks are larger than the supply leaks, sealing the return leaks but not the supply leaks can also cause unintended problems. In particular, the supply leak now becomes an unbalanced leak with the attendant house depressurization problems described above. Clearly, in the house as a system view, the ducts are not simply the components attached between the airhandler and the house. Instead, they also include the occupied spaces and envelope of the house. Residential commissioning can account for these systemic issues, but needs to be done on a whole-house basis rather than component by component. 


\section{What Needs to Be Commissioned?}

Many components and systems in a house can benefit substantially from commissioning. For simplicity and practicality, one might consider only evaluating individual components and systems. However, this approach is insufficient, because many of them interact. Therefore, to account for the interactions of individual components in the complete building system and to identify the energy and non-energy opportunities for improving the performance of the components, commissioning needs to be carried out on a whole-house basis. As a result, this guide focuses on areas of particular concern with significant interactions: building envelope and HVAC systems. These areas include insulation quality, windows, airtightness, envelope moisture, fan and duct system airflows, duct leakage, cooling equipment charge, and combustion appliance backdrafting with spillage.

- Building Envelope: The building envelope is important to the performance of a house, because envelope loads dominate the house heat transfer mechanisms. Assumed thermal loads, equipment sizing, structural durability, and occupant comfort for houses are based on having the building envelope perform as intended, including insulation levels, windows, and airtightness. In new houses, installation failures, especially in insulation and air sealing, can cause immediate energy consumption and comfort problems. As the building ages, subsequent loss of durability caused by poor material selection and installation (e.g., insulation settling, air barrier damage from UV exposure, moisture damage) can result in further performance reductions over time.

- Air Distribution Systems: Ducts that are part of the thermal distribution system may be the single worst performer in the energy performance of a house (Jump et al. 1996). Duct leakage, duct insulation compression, and other poor installation practices can reduce duct efficiency by $30 \%$ from even a moderate level of design performance. Compared to the space conditioning system, the ventilation system in most houses is simple. It consists of operable windows, infiltration, and a few (if any) intermittently-operated local exhaust fans. However, some houses use whole-house ventilation as well, sometimes directly linked to the space conditioning system. The delivery effectiveness and room by room distribution efficiency of both the thermal and ventilation distribution systems thus depends on the proper flow of air through the air moving equipment. Poor operation of the air distribution systems wastes energy and can cause comfort problems, structural moisture problems, and poor indoor environmental quality.

- Cooling Equipment: Even in new houses, cooling systems rarely perform as intended (Sherman et al. 1987). In particular, refrigerant charge levels and airflow across coils often do not meet manufacturers specifications used in the system design. As a result, the capacity and efficiency of the equipment can be substantially degraded.

- Combustion Appliances: Fueled appliances must vent as intended. Poor installation of either the combustion equipment or air moving equipment can reduce efficiency and also lead to backdrafting and combustion gas spillage or other hazards. Such events, along with insufficient ventilation for unvented combustion appliances, can directly affect the indoor environment and cause health or comfort problems.

Related to the performance of thermal and ventilation distribution systems is a host of indoor air quality (IAQ) issues. These issues include the generation, transport, and removal of pollutants, which are difficult to account for directly in commissioning. Although we recommend 
commissioning ventilation systems to deal with related transport and removal processes, there are numerous pollutant sources in every house and accounting for each is impractical during residential commissioning. Example sources are people, building materials, and combustion appliances that vent indoors. Example pollutants related to these sources include gaseous ones such as carbon monoxide and nitrogen oxides; biologically active ones such as molds, fungi, and mites; and particulates such as skin flakes and soot. As a result, we do not provide a separate section in this guide to discuss IAQ commissioning. Instead, our discussions of component commissioning describe IAQ issues when appropriate.

Although there are few controls in a house compared to those in commercial buildings, controls and occupant interactions with them can play an important role in house performance, especially when the systems become complex (e.g., multistage systems, integrated heat-pump/ventilation systems). Even common heating-setback / cooling-setup thermostats need to be properly commissioned and occupants need to be educated in their proper use. Making sure that these controls are doing what was intended or is appropriate is often crucial to achieving good energy performance. In this guide, we do not provide a separate section to discuss controls, because their effects are integrated with component and whole-house performance. Instead, our discussions of components describe control issues when appropriate.

Apart from the HVAC system, there are many other large appliances in the house. Some of them (e.g., stoves, water heaters, refrigerators, clothes dryers) can use considerable amounts of energy. Improper configuration of these appliances (e.g., a high temperature setting for the water heater, a clogged dryer vent) can cause poor performance. Most such appliances require only simple commissioning through visual inspection and comparisons to manufacturer recommendations or to occupant expectations. Except for appliance operation that might affect other components (e.g., room depressurization from dryer operation, which then leads to backdrafting of a combustion appliance), we do not discuss these appliances in this guide.

\section{What is Commissioning?}

Commissioning has its roots in shipbuilding, where the term describes the process that ensures a new vessel is sea worthy and ready for service. This process has many specific definitions. The variation relates to the scope of commissioning, and the activities related to it. Some commissioning projects begin early in the design stage and continue through ongoing operations and maintenance. Others include activities to optimize performance beyond design intent (supercommissioning) or to adjust performance of existing facilities (retro-commissioning).

In a very narrow sense, one can simply think of residential commissioning as a process only for new houses. This process would assure the owner that all required equipment is correctly installed, the final product is correctly assembled, and the house can perform as intended. Such a process might be carried out after installation and construction are complete and before the buyer occupies the new house.

For the purposes of this guide, we broaden our definition of residential commissioning to include many other activities such as auditing, rating, super-commissioning, or retro-commissioning. As such, it represents an expansion of processes currently carried out by people such as home energy raters, home inspectors, auditors, and weatherization contractors. This expansion includes the energy performance of the large number of existing houses, as well as the indoor environmental performance of all houses. 


\section{Characteristic Elements of Commissioning}

Residential commissioning, like every other commissioning process, includes three characteristic elements. The following defines these elements and offers examples to aid understanding:

Metrics: There are three broad whole-building objectives related to achieving a safe, healthy, comfortable, and affordable house that has a minimal adverse impact on the external environment:

- energy performance,

- indoor environmental performance, and

- durability.

Various qualitative or quantitative metrics can represent each objective.

- For energy related to providing building services, they include fuel consumption, peak demand, and operating costs.

- For the indoor environment, they include indoor air quality and comfort.

- For durability, they include material degradation due to moisture (other causes of degradation include sunshine, earthquakes, and fire, but we only deal with moisture in this guide) and maintenance costs.

Each metric is simply a scale to rank the performance element of interest. Metrics can also represent the performance of building components or systems. Three examples of such metrics are:

- $\quad$ specific leakage area, which is a metric for the airtightness of the building envelope;

- duct leakage, which is a metric for the air leakage from a duct system; and

- house depressurization, which is a metric for the backdrafting potential of combustion appliances.

Relationships between metrics need to be considered, due to the energy, indoor environmental, and durability performance interactions between components and systems (Koles et al. 1996). For example, it is necessary to quantify specific leakage area, duct leakage, and house depressurization to understand the impact that reducing duct leakage flows to save energy can have on combustion safety in tight houses.

Diagnostics: Diagnostics are usually defined as relatively quick short-term field procedures. These procedures involve measurements and perhaps analyses to evaluate performance metrics for a system or component under functional test or actual building site conditions.

An example of a diagnostic is the use of airflow measuring equipment that creates and measures pressure differences, which one can then use in subsequent computerized analyses to calculate duct leakage.

While it is also possible and sometimes preferable to evaluate metrics using data taken over an entire season, time limitations make it impractical to collect and analyze such long-term information during residential commissioning. These limitations will largely depend on the value of the commissioning process to the involved parties. However, for an existing house, 
commissioning can often use readily available historical data either as part of diagnostics or to set norms.

Norms: A norm is a benchmark or set of benchmarks against which one compares component, system, or whole-building performance. Examples of norms are the various building standards that specify requirements for minimum or maximum specific leakage area, for maximum duct leakage, and for maximum house depressurization levels. A specific example is the California Title 24 norm that duct leakage be $6 \%$ or less of the nominal total airflow through the air handler.

New and existing houses can use the same metrics and diagnostics, although some diagnostics may not be appropriate at all stages of the construction process. However, the norms for existing houses will have to be adjusted to account for the stage of the house in its life-cycle and the economic viability of meeting stricter standards than those in place at the time of construction. For example, a house built in 1930 does not come close to meeting current energy consumption limits. The retrofitting required to meet new insulation level requirements in this example would be prohibitively expensive.

\section{The Commissioning Process}

Many in the building industry think commissioning applies only to commercial buildings, even though it is still uncommon to commission these buildings at any stage of their life cycle. Most descriptions of commercial building commissioning (Wray et al. 2000) include the following three general steps:

- develop a commissioning plan,

- carry out inspections and functional performance tests, and

- review operations, maintenance, and training procedures.

Commissioning processes for houses are different. One reason is that commercial buildings tend to be unique compared to one another, are large, have complex control systems, and have personnel that manage operations and maintenance (O\&M). As a result, a common step in the commissioning process for these buildings is developing unique documentation: a distinct commissioning plan and a building-specific O\&M manual. This step is not warranted for most houses, because they tend to be more similar compared to one another, are small, have few control systems, and have no O\&M personnel. Another reason is that we anticipate residential commissioning can sometimes provide better performance than is called for in the design; most commercial commissioning ignores this goal.

The residential commissioning process that we envision has three main phases that can probably be encompassed in generic guidelines for specific commissioning issues or system and component types:

- audit and diagnostic,

- tuning and tweaking, and

- opportunity identification.

The following describes these three phases. 
Audit and Diagnostic: The first phase of commissioning uses audit and diagnostic techniques to survey metrics for the house and then compares survey results with appropriate norms for the house.

For new construction, the norms will be those such as California Title 24 standards or the equivalent local building codes.

For an existing house, one might base the norms on design intents (if any were ever documented). Alternatively, one might base them upon what a particular component should be able to do relative to those in other similar houses.

Tuning and Tweaking: The performance of many components and systems may not meet the norms, but it may be possible to improve their performance immediately by making minor adjustments, repairs, or retrofits on the spot.

Examples include sealing leaky ducts or correcting a refrigerant charge deficiency in a central air conditioner. Such tuning and tweaking can often provide significant improvements in performance for very little marginal cost.

The purpose of this step is to improve the performance of the house to at least the design intent. Sometimes, that intent will be unknown. In those cases, the optimization will be to other norms, such as the best performance achievable within cost limits.

Opportunity Identification: After the tuning and tweaking, there still may be components that are not performing up to their potential. This commissioning step provides the client with information about what potential repair or retrofit opportunities need further consideration. Even when components are performing to their norms, the improved performance of new components or systems may make replacement worthwhile.

Examples include adding insulation to areas such as the ceiling, walls, and floors; replacing windows that a homeowner wishes to change anyway for aesthetic reasons or to solve comfort, condensation, or maintenance problems; and replacing an oversized inefficient cooling or heating system that is near the end of its service life with a properly sized and more efficient system.

\section{Overcoming the Commissioning Cost Barrier}

A barrier to commissioning is that the building industry and the consumer currently view the process as a cost, without sufficient perceived compensating benefit. Because of the emphasis that these parties place on first cost, such a view has resulted in little demand and effort to provide commissioning services. To overcome this barrier, it is important to not only provide an integrated set of simple, rapid, inexpensive, and reliable commissioning methods, but also to quantify the potential benefits of commissioning.

\section{Cost of Commissioning}

The cost of commissioning will be highly variable. It will depend on the specific implementation of a commissioning program and will depend on how commissioning is folded in with other programs. It will also depend on the training of the personnel and on the level of commissioning chosen. Commissioning could be a loss leader, a built-in cost, a profit center, or part of a public purpose program or regulation. Costs may also change in the future as residential commissioning activities increase. 
Because of all this variability, dollar-cost estimates for commissioning are difficult to establish at this time. However, we can indicate some ranges of required resources. Testing a house as part of the commissioning described in this guide should take a trained crew from 4 to 6 personhours, excluding tuning and tweaking of the building and its systems. The amount of specialpurpose equipment required for the tests would cost between $\$ 6,000$ and $\$ 15,000$. The biggest variation in the total cost comes from using additional equipment to automatically control duct leakage and grille airflow tests. Together, a data acquisition system and computer for this control cost about $\$ 3,000$ to $\$ 5,000$. Automatic control is not necessary to carry out these tests, but tests without it will take about 50\% longer (6 hours rather than 4 hours). With automatic control, it is likely that a two-person commissioning team could test two houses a day, excluding travel time. Another large variation in the total cost is the price of blower doors: they currently cost about $\$ 1,600$ to $\$ 3,500$.

\section{Tunneling through the Cost Barrier}

A whole-house commissioning approach can maximize the return on investment from commissioning and allow houses to reach the next level of performance - beyond that of the current Title 24. In doing so, it "tunnels through the cost barrier" (Rocky Mountain Institute 1997, Lovins 1999) to the next level for new construction, as well as for the retrofit of existing buildings. While the performance expectations are clearly different for each set of houses, the approach is similar.

To understand the cost barrier, consider the situation in which a set of energy-efficiency construction options or retrofits is prioritized through design or commissioning by return on investment (ROI). Starting with the highest ROI measure and adding individual measures in descending order will eventually mean that the available budget is expended or that the incremental ROI for each additional measure is so small that it is not worth implementing. At this point, the cost effectiveness limit has been reached. However, if additional issues such as capital cost, maintenance cost, risk, and comfort are included, or if a retrofit is carried out simultaneously with other renovations that would have been done anyway, it is possible to go beyond this artificial cost barrier. In these cases, the resulting system will be more energyefficient at a lower total cost as a whole than the individual components alone would dictate. This approach only works if the house is considered as a system and if commissioning is used to ensure that the benefits can indeed be captured. It involves saving more capital cost from eliminated loads in an integrated system than was paid to eliminate the loads. Integration allows energy-efficiency measures that make little sense individually (e.g., windows) to be costeffective and attractive together within the whole system, due to concurrent benefits such as reduced equipment size and improved comfort.

\section{Potential Benefits of Commissioning}

Matson et al. (2002) have assessed the potential benefits of whole-house commissioning for new and existing coastal and inland valley California houses. In this assessment, tuning and tweaking houses as part of commissioning included tightening the building envelope, tightening and insulating ducts, and correcting refrigerant charge and HVAC system airflow deficiencies. For new Title 24 houses, this phase of commissioning also included correcting insulation and window problems, because builders can more easily do this when tuning and tweaking activities identify such problems during construction. Existing houses and new Title 24 houses offer further opportunities for significant changes beyond tuning and tweaking. In the assessment, these opportunities included installing more efficient HVAC equipment for both house types, 
adding insulation to the existing houses, and upgrading the windows in the existing houses. Advanced houses included in the assessment do not have these additional opportunities and tend not to need insulation corrections, because they are well engineered and use technologies less prone to insulation quality problems.

- The magnitude of potential benefits from whole-house commissioning varies widely, depending on climate, house type, equipment type, and the types of improvement opportunities available. Benefits range from almost nothing in some well-engineered advanced new houses to large savings in poorly-performing existing houses: as much as $80 \%$ savings in HVAC electricity consumption, 70\% savings in HVAC natural gas consumption, $70 \% \mathrm{HVAC}$ operating cost savings, and $80 \%$ reductions in total HVAC fuel-related carbon emissions. Interestingly, the potential energy savings from tuning and tweaking new Title 24 houses are similar to the results of capturing all available opportunities in smaller existing houses. For typical houses, the potential savings are only about $1 / 3$ to $1 / 2$ of the savings in the poorly-performing houses, because the typical houses have fewer opportunities for change.

- Qualitatively, commissioning offers a broad spectrum of potential long-term benefits for various stakeholders such as builders, consumers, code officials, utilities, state agencies, and energy planners. Builders and/or commissioning agents will be able to improve system performance and reduce consumer costs associated with building energy use. Consumers will be more likely to get what they paid for and builders can show they delivered what consumers expected. Reduced callbacks and warranty costs will provide the business community with increased profits. Code officials will be better able to enforce existing and future energy codes. As energy reduction measures are more effectively incorporated into the housing stock, utilities and energy planners will benefit through greater confidence in predicting energy use and greater assurance that energy efficiency reductions will actually occur. Performance improvements will also reduce emissions from electricity generating plants and residential combustion equipment, which will benefit the environment as a whole. As the commissioning industry expands, a larger workforce will be required, which will lead to new jobs that benefit the state and the economy.

\section{Reducing the Need for Commissioning}

One way to reduce commissioning costs is to build a house that does not need as much commissioning. For example, production builders outside California have recently used the "house as a system" approach in advanced houses to substantially improve house performance without changing the appearance of the house and with little or no additional construction cost increase compared to conventional construction. In particular, Pulte Homes in Las Vegas, together with Building Science Corporation and the DOE Building America program, have implemented a system involving several integrated key changes to conventional construction (ESB 1997, HEM 1998, Building America 2000). These changes include:

- Moving insulation from the ceiling to the roofline, eliminating attic venting, and centrally locating the air-distribution system. The ducts remain in the attic, but they are now located within the conditioned thermal envelope rather than in an unconditioned hot attic. In addition, the central location means that shorter duct runs are required. These changes increase cooling efficiency, save energy, and increase comfort. They also save on duct material and installation costs, because it is now less important from an energy standpoint to 
seal and insulate the shorter ducts as much as in a conventional attic. Side benefits of eliminating the attic vents are reduced construction cost and reduced attic infiltration.

- Changing framing from $2 \times 4$ to $2 \times 6$ studs and reducing the number of framing members in the building envelope by $30 \%$ (Building America 2000). This change reduces construction cost (fewer materials and less labor for construction) and enhances the building strength. It also reduces the thermal load on the cooling system, because it reduces the area of thermal bridges and allows more insulation to be installed between studs.

- Extensive air sealing of the envelope, which includes a double air-barrier system. This system uses rigid foam insulation as the sheathing in place of oriented-strand board (OSB) and building paper or house wrap. The result is reduced construction cost, better rain control, reduced infiltration, reduced thermal loads, and improved building durability.

- Reducing the cooling capacity of the cooling system to $60 \%$ of the size required without other improvements, such as from a 2.5 ton unit to a 1.5 ton unit. This change can be made because thermal loads are reduced. A benefit is that the first cost of the smaller system is lower (savings are on the order of $\$ 300$ to $\$ 650$ per ton, ESB 1997) and it is less expensive to operate.

- Cost savings associated with the other changes are used to pay for improved windows (vinyl frames with low-e double glazing) and a controlled whole-house mechanical ventilation system. Benefits of these changes are further reductions in thermal loads, improved comfort, reduced ultraviolet light damage to interior surfaces, reduced window condensation, and improved and more reliable indoor air quality.

For the builder, benefits associated with the house as a system approach described above include improved work crew productivity, reduced commissioning needs, reduced callbacks, and the ability to sell the house for more than the increase in cost. For the homeowner, in addition to improved comfort and a two-year guarantee of reduced energy consumption (about $30 \%$ compared to Model Energy Code requirements, LVR-J 2000), benefits include improved affordability and greater financing options, because the utility bills are more predictable. Other benefits include reduced maintenance costs and increased resale value.

\section{Qualified Commissioning Agents}

A common question regarding commissioning is "Who is qualified and who should do it?" The most common method for commissioning commercial buildings today is to hire an independent third party (Commissioning Authority) to lead the commissioning effort. The independence allows the Commissioning Authority to maintain neutrality and avoid conflicts of interest, which is difficult if the design team also does the commissioning.

In spite of the benefits of independence, many design engineers argue that they are the best qualified to conduct the commissioning. One reason is that they are closest to the design. A second is that they understand the functional intent. A third reason is that they believe they should be involved in defining and performing test sequences. A problem with this arrangement is that the design team is less interested in uncovering design problems that an independent party might more fully explore.

Even when the Commissioning Authority is an independent third party, the job can be complicated by design problems. For example, the Commissioning Authority is supposed to 
ensure that the installed system functions in an optimal fashion, but is in a quandary when problems with the original design are found after installation. Commissioning Authorities are not usually responsible for the design. Therefore, to facilitate feedback to the designers on how building systems actually perform, the Commissioning Authority should be engaged early in the process.

Other problems can arise in commissioning when the Authority does not become involved until late during the design or early in construction. An example is that the collection of information (such as design specifications and drawings) required to perform commissioning is more difficult later in the process. Another example is that late scheduling of tests into a typically rushed and inflexible construction and start-up schedule is more difficult and therefore more expensive.

Many of these principles apply to houses as well, even though they are not typically "designed". In addition to builders and their subcontractors, likely parties who will be directly involved in residential commissioning include the State through statewide energy codes, home energy auditors and raters, home inspectors, and weatherization contractors. Other parties involved may include utilities, realtors, financial and insurance institutions, and environmental groups.

Within California, there are several active new construction and home retrofit energy-efficiency programs, but few people are currently qualified to commission houses on a whole-house basis (Knight and Thomas 2000, Wirtshafter Associates 2000). Perhaps the best qualified at this time are home energy raters and building performance contractors, who typically have more building science training and a better understanding of the house-as-a-system concept than any other group. If these independent parties are used to commission houses, we envision that builders and perhaps subcontractors may still carry out some commissioning themselves throughout the construction process in preparation for the independent commissioning. However, careful integration of the building team and independent party commissioning with the construction process is important to avoid scheduling conflicts and unnecessary cost. Involving independent parties in the construction process has been problematic in the past (Pacific Consulting Services 2000). For whole-house residential commissioning programs to succeed, training, certification, accreditation, and commissioning management processes need to be developed and widely used. We anticipate that involving state agencies and building industry associations in the development of such processes is key. 


\section{COMMISSIONING APPLICATIONS}

This section provides two examples that demonstrate the application of whole-house commissioning to new and existing California houses. In each example, we first summarize problems that diagnostic testing could find. We then describe the corrections needed to improve house performance: "on the spot" tuning and tweaking changes to correct as many problems as possible, and then the opportunities for further changes. At each correction step, we also describe the benefits of making the changes. These benefits include HVAC electricity consumption savings, natural gas consumption savings, operating cost savings, and carbon emission savings.

The two examples are extracted from the set of hypothetical California houses simulated in the potential benefits analysis of Matson et al. (2002). In particular, we use the typical pre-Title 24 existing and Title 24 houses located in Sacramento. "Typical" represents our assessment of the penetration of individual energy efficiency measures within California houses. Therefore, the savings that we report in our examples represent typical combinations of problems, which assume some houses do not have all the problems that we list. If all the problems that we list in our examples occurred together, the savings from commissioning these houses would be even greater than what we report here. Field demonstrations are needed to confirm that these benefits can be achieved by applying whole-house commissioning to new and existing California houses.

There are various associated qualitative benefits from applying whole-house commissioning to these houses, which are discussed by Matson et al. (2002), but not here.

\section{New House Example}

This example describes a hypothetical 2,500 $\mathrm{ft}^{2}$, two-story, four-bedroom wood-frame house with an uninsulated slab-on-grade foundation. It was to be built according to Title 24 Package D minimums. Commissioning starts during the construction process, which allows problems to be found and corrected early.

\section{As Found}

Commissioning this new house finds several problems:

1. The walls have nominal R-19 fiberglass insulation and $25 \%$ framing, but the net wall thermal resistance is R-11.5 rather than the expected R-13.8, due to $30 \%$ insulation void area found by visual inspection.

2. The ceiling has nominal R-38 blown cellulose insulation and $10 \%$ framing, but the net ceiling thermal resistance is R-22.3 rather than the expected R-37.5, due to $2.5 \%$ insulation void area and 20\% fluffing loss found by visual inspection. Fluffing is defined as installing the insulation so that it appears to be thick enough but its density is insufficient.

3. Visual inspection and a glazing emittance classification test shows that the windows are clear double-pane glazing mounted in aluminum-frames (no thermal break) instead of the expected low-solar-gain low-e double-pane glazing mounted in vinyl frames.

4. Based on a blower door test, the envelope has a normalized leakage area (NL) of 0.75 (SLA of 6.1), which is much leakier than the expected NL of 0.5 (SLA of 4.1). Using ASHRAE Standard 136, the effective ventilation rate for this house as found is $0.57 \mathrm{ach}$, which is almost double what ASHRAE Standard 62.2P requires for this house ( $0.34 \mathrm{ach})$. The house will have too much ventilation. 
5. A duct leakage test indicates the ducts have $22 \%$ total leakage to outside (split evenly between the supply and return), instead of the expected $6 \%$ total leakage.

6. Visual inspection shows the natural-gas-fired furnace has an AFUE rating of $80 \%$ rather than the expected $90 \%$.

7. Based on a visual inspection, the cooling system has the required thermostatic expansion valve (TXV) refrigerant control. A subcooling test indicates the cooling system is $15 \%$ undercharged, and an air-handler airflow test shows that it only has $85 \%$ of its required evaporator airflow (resulting in $340 \mathrm{cfm} /$ ton instead of $400 \mathrm{cfm} / \mathrm{ton}$ ). There is a standard fan motor for the air-handler instead of a more efficient ECM motor.

\section{Tuning and Tweaking}

By correcting problems during construction so that components meet their expected norms (correcting insulation problems in the walls and ceiling, installing the correct windows, sealing the envelope and ducts, charging the cooling system properly, and adjusting the air-handler airflow), our simulations indicate that tuning and tweaking this new house can capture the following savings:

1. $200 \mathrm{kWh} / \mathrm{yr}(9 \%)$ HVAC electricity consumption savings,

2. 110 therms/yr (24\%) HVAC natural gas consumption savings,

3. $\$ 90 / \mathrm{yr}(17 \%)$ HVAC operating cost savings, and

4. $580 \mathrm{~kg} / \mathrm{yr}(26 \%) \mathrm{HVAC}$ carbon emission savings.

These changes also lower the effective ventilation rate of the house to $0.39 \mathrm{ach}$, which is much closer to the 0.34 ach rate required by ASHRAE Standard 62.2P for this house.

\section{Opportunity Identification}

In this new house, the AFUE and motor problem can be corrected by replacing the furnace with the expected equipment (90\% AFUE with an ECM motor). These changes can result in additional savings. Our simulations indicate that the total savings captured by commissioning with these additional opportunities are:

1. $230 \mathrm{kWh} / \mathrm{yr}(11 \%) \mathrm{HVAC}$ electricity consumption savings,

2. 140 therms/yr (31\%) HVAC natural gas consumption savings,

3. $\$ 110 / \mathrm{yr}(22 \%)$ HVAC operating cost savings, and

4. $750 \mathrm{~kg} / \mathrm{yr}(33 \%) \mathrm{HVAC}$ carbon emission savings.

These changes will not lower the effective ventilation rate of the house.

\section{Existing House Example}

This example describes a hypothetical 1970's pre-Title $241,455 \mathrm{ft}^{2}$, one-story, three-bedroom wood-frame house and an uninsulated slab-on-grade foundation. In this case, commissioning occurs many years after construction, which means some problems like insulation deficiencies are more difficult to detect and more effort is required to correct the problems now. 


\section{As Found}

Commissioning this existing house finds several problems:

1. The walls have no insulation, based on probing stud cavities in places such as closets, where it is easier to repair the holes made for probing. The wall's 2"x4" framing will limit the amount of insulation that can be placed in the wall cavities.

2. The ceiling has nominal R-30 blown cellulose insulation and $10 \%$ framing, but the net ceiling thermal resistance is R-18.5 rather than the expected R-29.5, due to $2.5 \%$ insulation void area and $20 \%$ fluffing loss found by visual inspection.

3. Visual inspection shows that the windows are clear single-pane glazing mounted in aluminum-frames (no thermal break).

4. Based on a blower door test, the envelope has a normalized leakage area (NL) of 1.2 (SLA of 12), which is much leakier than the NL needed to ventilate the house by infiltration alone (0.49, SLA of 4.9). Using ASHRAE Standard 136, the effective ventilation rate for this house as found is $0.93 \mathrm{ach}$, which is more than double the rate that ASHRAE Standard 62.2P requires for this house $(0.38 \mathrm{ach})$. The house will have too much ventilation.

5. A duct leakage test indicates the ducts have $28 \%$ total leakage to outside (split evenly between the supply and return). This is much leakier than the total leakage that can be achieved by duct sealing (6\%). Visual inspection shows that the ducts are uninsulated.

6. Visual inspection shows the natural-gas-fired furnace has an AFUE rating of only $78 \%$.

7. Based on a visual inspection, the cooling system has a capillary-tube refrigerant control. A superheat test indicates the cooling system is $15 \%$ undercharged, and an air-handler airflow test shows that it only has $85 \%$ of its required evaporator airflow (resulting in $340 \mathrm{cfm} / \mathrm{ton}$ instead of $400 \mathrm{cfm} / \mathrm{ton})$. There is a standard fan motor for the air-handler instead of a more efficient ECM motor.

\section{Tuning and Tweaking}

Tuning and tweaking this existing house is limited to sealing the envelope, insulating and sealing the ducts, charging the cooling system properly, and adjusting the air-handler airflow. Other improvements require more effort and must be left to the opportunity identification phase of commissioning. Our simulations indicate that the following savings can be captured:

1. $540 \mathrm{kWh} / \mathrm{yr}(16 \%) \mathrm{HVAC}$ electricity consumption savings,

2. 110 therms/yr (21\%) HVAC natural gas consumption savings,

3. $\$ 130 / \mathrm{yr}(18 \%)$ HVAC operating cost savings, and

4. $610 \mathrm{~kg} / \mathrm{yr}(23 \%) \mathrm{HVAC}$ carbon emission savings.

These changes also lower the effective ventilation rate of the house to $0.39 \mathrm{ach}$, which is much closer to the 0.38 ach rate required by ASHRAE Standard 62.2P for this house.

\section{Opportunity Identification}

In this existing house, there are major opportunities to improve house performance beyond tuning and tweaking. These include: 
1. Insulating the empty wall cavities with nominal R11 blown cellulose insulation (net wall thermal resistance becomes R-9.1).

2. Adding more blown cellulose insulation to the ceiling to achieve a nominal R-38 level (net ceiling thermal resistance becomes R-37.5).

3. Replacing old windows with new low-solar-gain low-e double-pane vinyl-frame windows.

4. Replacing the existing heating system with a more efficient (90\% AFUE) furnace equipped with an ECM motor, and replacing the evaporator with one equipped with TXV control.

Our simulations indicate that there are considerable total savings captured by commissioning with these additional opportunities. They are:

1. $2,240 \mathrm{kWh} / \mathrm{yr}(67 \%) \mathrm{HVAC}$ electricity consumption savings,

2. 270 therms/yr (54\%) HVAC natural gas consumption savings,

3. $\$ 410 / \mathrm{yr}(61 \%)$ HVAC operating cost savings, and

4. $1,640 \mathrm{~kg} / \mathrm{yr}(63 \%)$ HVAC carbon emission savings.

These changes will not lower the effective ventilation rate of the house. 


\section{COMMISSIONING COMPONENTS}

\section{Building Envelope}

The building envelope includes the floors, walls (including windows and doors), ceiling, and roof of the building. It serves as the building structure and is a heat, airflow, moisture, and pollutant retarder between outdoors and indoors. The envelope is important to house performance, because heating and cooling loads are dominated by heat and moisture transfer through the envelope. HVAC system loads, equipment sizing, structural durability, and occupant comfort are all based on having the envelope perform as intended.

\section{Insulation Quality}

\section{Introduction}

The purpose of envelope insulation is to reduce heat transfer across opaque building envelope elements and to control assembly temperatures for comfort and for avoiding condensation. The amount of insulation that is appropriate depends on how severe the climate is, on the cost of materials and installation, and on the building operating costs.

In new houses, installation failures cause immediate problems. Christian and Kosny (1998) indicate that insulation installation deficiencies can increase whole-wall heat transfer by about $14 \%$, which will increase energy consumption and reduce comfort. Examples of deficiencies in California houses include missing or compressed fiberglass insulation in walls. Based on a survey of 24 California houses, Davis Energy Group (2000) found that such deficiencies on average affect about $8 \%$ of the net wall area (excludes windows and doors) and reduce the cavity nominal thermal resistance by about $30 \%$, with extreme cases affecting about $20 \%$ of the net wall area together with almost 50\% thermal resistance reductions. Particular areas of concern to focus on in commissioning are knee walls, skylight shafts, and areas with electrical or plumbing services. Even greater problems can occur for ceilings due to the large temperature differentials between the attic and house in conjunction with ceiling insulation deficiencies, such as completely uninsulated regions or "fluffing" the insulation to look thicker (Uniacke 2000, Davis Energy Group 2000).

Compounding these problems, as the building ages, loss of durability caused by poor material selection and installation (e.g., insulation settling, air barrier damage from pre-installation UV exposure, water intrusion) can result in further performance reductions in existing houses.

\section{Applicable Metrics}

Insulation "level", location, and framing fraction are simple metrics for opaque envelope elements. The insulation "level" can be defined in terms of its type, thickness, and/or density. Framing fraction is defined as the amount of thermal bridging between insulation cavities relative to the total envelope element area. Other related qualitative metrics include the presence of anomalies such as missing insulation, insulation settling in a wall, or uneven distribution over a ceiling. Because these metrics can be easily determined through visual inspection during construction, we recommend that they be the primary metrics for use in commissioning. Due to the lack of practical functional performance diagnostics, we suggest that these metrics also be used for existing houses, even though it is much more difficult to determine them through visual inspection in such houses. 
Two common metrics that are more quantitative are the thermal conductance or resistance of opaque elements, respectively denoted by the assembly U-value (heat loss or gain per unit area and per unit temperature difference) and assembly R-value (inverse of U-value). Christian and Kosny (1995) have refined the conductance metric for wall sections using terms such as centerof-cavity (not including framing or additional elements such as doors or windows), clear-wall (including framing but no additional elements such as doors or windows), and whole-wall conductance (including framing, doors, and windows). Even though building requirements often define norms for these metrics, we do not recommend their use in commissioning because they are practically unverifiable in real houses.

\section{Recommended Diagnostics}

The literature that we reviewed described several quantitative techniques for evaluating the thermal performance of insulation systems (Wray et al. 2000). However, due to measurement biases in some cases, lack of portability or commercial availability in other cases, the need for expert users, excessive time requirements, and expensive equipment, these diagnostics are impractical in a residential commissioning environment. As a result, qualitative visual inspections will often play a key role instead, especially in new houses during construction. Qualitative infrared inspection can be used as a supplementary diagnostic in existing houses to support visual inspections, but is severely restricted in its applicability due to environmental constraints.

- Visual Inspection: Visual inspection (Consol 1999) determines the presence or absence of deficiencies such as incorrect framing, incorrect insulation type, incorrectly installed insulation, incorrect air and vapor barrier placement, barrier damage, and blocked ventilation pathways (e.g., between attic and soffitt vents).

Such inspections are simpler and most useful when carried out during construction. The inspection of building assemblies should happen before construction crews seal the framing and insulation from view, while it is easier to correct problems. In existing houses, it is often difficult or impossible to inspect these assemblies without probing or dismantling exterior or interior surfaces of the assembly. Other visual inspection steps can include checking that labels from each insulation bag are stapled in the attic. The presence of these labels helps ensure that the correct total amount of insulation is installed, but not how it has been distributed.

Visual inspections during construction require no equipment other than a ruler or tape measure, and possibly a flashlight. Common building construction tools to dismantle and repair assemblies may be required to inspect insulation in existing houses.

These inspections do not quantify assembly thermal resistance or conductance (R- or Uvalue). However, one can subsequently calculate assembly thermal performance using data from these inspections in conjunction with building plans or an assembly material audit. Well-developed commercially available software can facilitate these calculations. Some of this software can also predict thermal parameters such as local and average surface temperatures, as well as heat loss or gain rates. Unfortunately, most of this software is intended for research use rather than design or commissioning. Current efforts sponsored by the ASHRAE Technical Committee on Energy Calculations to create simplified analysis methods may help alleviate this shortcoming. 
- Infrared Inspection: Infrared thermography systems and thermometers (radiometers) detect long-wave radiation during a scan of a building assembly and convert the long-wave radiation signal to a surface temperature using a fixed surface emissivity. Most thermography systems and scanning radiometers produce false color images of the scan on a video display for easier viewing of intensity differences. Scanning thermography equipment is expensive: the cost of a new system is in the range of $\$ 12,000$ to $\$ 60,000$. "Point source" radiometers are substantially less expensive ( $\$ 200$ to $\$ 1,300)$. They simply display a spot-area-average temperature as a meter reading. The spot area increases as the distance from the surface of interest increases. This device provides a crude screening mechanism to rapidly locate envelope element deficiencies.

The field use of any infrared inspection technique (ASTM 1990) is limited and may not be repeatable for several reasons. These include: equipment thermal instability at very high or very low outdoor temperatures; poor resolution at low indoor-outdoor temperature differences; and distortion of surface temperature patterns by solar radiation and wind effects, differential heating or cooling rates of dry and wet areas, and non-uniform or specular emissivities. Infrared inspection generally is not useful for quantifying assembly thermal conductance or even framing fractions (Davis Energy Group 2000).

Within the limitations described above, infrared inspection can qualitatively identify areas of anomalous heat transfer caused by design and/or construction deficiencies of the building envelope. Examples include lack of insulation, displaced or improperly installed insulation, wet insulation, thermal bridging, air leakage, and air intrusion. Pressurizing or depressurizing the building using a blower door during a thermographic scan can highlight air leakage paths that might otherwise be confused as insulation anomalies. Thermographic scanning can also be useful in identifying envelope sections with internal convective loops.

\section{Procedural References}

1. ConSol. 1999. "CIEE Final Project Report: Protocols for Energy Efficient Residential Building Envelopes”. Stockton, CA; ConSol. Also available from the California Energy Commission at http://www.energy.ca.gov/efficiency/qualityhomes/protocols.html.

2. ASTM. 1990. "ASTM Standard C1060-90, Standard Practice for Thermographic Inspection of Insulation Installations in Envelope Cavities of Frame Buildings". Philadelphia, PA; American Society for Testing and Materials. http://www.astm.org.

\section{Windows}

\section{$\underline{\text { Introduction }}$}

The primary purpose of windows is to allow occupants to see outdoors. They also serve as a light source, as an aperture for solar heat gains (desirable during the heating season, but undesirable during the cooling season), and as openings (if operable) for ventilation and cooling. With the exception of some advanced windows that are not commonly installed, window thermal resistance is much lower than that of opaque elements. Because of these characteristics, windows can be one of the largest contributors to heating and cooling loads in a house. In addition, during the heating season, the use of an inappropriate window can lead to low temperatures on the window's interior surfaces, which in turn can cause thermal comfort and indoor air quality problems (e.g., increased radiative heat loss from occupants to nearby cool window surfaces, 
biological growth due to condensation on windows). As a result, having an appropriate window type installed correctly is important.

For example, Carmody et al. (2000) indicate that the thermal conductance and solar heat gain coefficient (SHGC) for a double-glazed window can each be reduced about $60 \%$ by using a lowsolar-gain low-emittance (low-e) coating and a vinyl frame, compared to using clear glazing and an aluminum frame. In turn, this can reduce the peak-cooling load for a typical southern-climate house by about $25 \%$. Glazing emittance and the location of the low-e coating (on the inside surface of the outer pane) are the most important contributors to this difference.

In spite of the importance of these factors, mislabeled windows are still installed in some new California houses. A recent survey involving about 110 houses (approximately 2,800 windows) found on average that about $3 \%$ of the windows are mislabeled (ConSol 2000). In two of the houses, as many as $17 \%$ of their windows were mislabeled. The mislabeling occurs during window manufacturing and is related to placing the low-e coating on the wrong pane (increases SHGC by about $20 \%$ ) or to having clear glazing instead of low-solar-gain low-e glazing. Without diagnostic tools, it is extremely difficult to visually detect emittance problems.

ASHRAE (2001a) indicates that inter-pane gap thickness also has a substantial effect on window conductance. For example, the conductance of a low-solar-gain low-e double-glazed vinylframed window with a 1/2-inch air- or argon-filled gap is about $15 \%$ lower than with a 1/4-inch gap. However, there appears to be no documented evidence that builders are installing windows with other than the requested gap.

\section{Applicable Metrics}

New windows have labels to list window characteristics (e.g., the National Fenestration Rating Council label). Metrics listed that describe a window's radiative behavior include emittance, solar heat gain coefficient (SHGC), daylight transmittance, and UV transmittance. These radiative metrics are simple to determine in the field and commercially available diagnostic tools are available for this purpose. Because of the considerable impact of window emittance on building energy use, we recommend that window diagnostics emphasize evaluating this metric.

Like opaque envelope elements, windows also use the conductance metric, but the lack of a practical conductance diagnostic makes it difficult to actually determine in the field. As a result, we recommend that commissioning should exclude the conductance metric at this time.

However, we recommend inter-pane gap thickness checks be carried out at the same time as emittance checks, because gap thickness is important to conductance, the diagnostic process is simple, the commercially available tools are inexpensive, and these tools are needed anyway to assess low-e coating location.

\section{Recommended Diagnostics}

Until recently, there were no practical field diagnostics to evaluate window properties and radiative behavior (e.g., glazing gap thickness, emittance, solar heat gain coefficient, daylight transmittance, and UV transmittance). A few inexpensive (\$130 to \$700) quantitative tools are now commercially available and more tools are under development. These tools can be used to evaluate windows in new and existing houses. They are most useful during construction (ideally used before the windows are installed), when it is less expensive to replace an incorrect window.

Note that using non-contact infrared thermography techniques in the field to assess window performance is generally impractical, due to radiation transmission and reflection effects. The 
magnitude of these effects can be highly variable depending on the physical properties of the glass, the presence of surface coatings, and the surface finish.

- Emittance Classification: A prototype handheld spectrometer can be used to determine window emittance class (Griffith 1999). This surface-contact tool uses an infrared emitter and detector to evaluate the aggregate normal reflectance of a multi-pane glazing assembly. Simple LEDs indicate whether the glazing is clear, has a high-solar-gain (low-e) coating, or has a low-solar-gain (spectrally-selective low-e) coating. The spectrometer works best when used on the pane with the coating, but it cannot distinguish which pane has the coating.

Commercially-available solar transmission meters are also available to determine window emittance class (EDTM 2000). However, they are less direct and more difficult to use than the handheld spectrometer, because they require measuring the solar irradiance with and without the glazing in the irradiance path. This can be difficult for fixed (non-opening) windows.

Gap Measurement and Low-E Coating Location: Other tools that use laser reflection can measure glazing gap thickness and distinguish which pane has the low-e coating (EDTM 2000). A digital numeric display or visible laser reflections on a sliding scale indicate gap thickness and which pane has the coating.

\section{Procedural References}

Although there are existing standards for measuring window emittance (e.g., NFRC 301-1993), they focus on laboratory procedures and are not practical for field use. There appear to be no standards for measuring the inter-pane gap or determining the low-e coating location. As a result, commissioning must rely upon procedures defined by manufacturers of window diagnostic tools.

\section{Airtightness}

\section{Introduction}

Air leakage through holes in the building envelope occurs due to indoor-outdoor pressure differentials created by stack effects (combined temperature and height differences), wind effects, and the operation of mechanical devices that move air between indoors and outdoors. The mechanically-induced airflows can be deliberate (e.g., a bathroom exhaust fan) or unintentional (e.g., supply duct leaks to outside).

Historically, homes were ventilated using a mixture of infiltration from leaky envelopes and occupant-controlled openings (i.e., windows). Until the energy crisis of the 1970s, many people thought this ventilation was sufficient, and there was less concern about the residential indoor environment. Today, people expect their house to isolate them from its surroundings and are more concerned about indoor air quality. Consequently, house envelopes have become tighter, proper ventilation is more necessary now, and mechanical systems may be installed in attempts to meet this need.

Installing mechanical ventilation systems in houses means that the designer needs to know the envelope air leakage. If the house turns out to be leakier than assumed, the house will be overventilated, which will unnecessarily increase building thermal loads. In this case, if the mechanical ventilation system is not balanced, then larger than expected infiltration might cause uncomfortable drafts during the heating season. If instead the house turns out to be tighter than assumed, the lack of leakage can lead to inadequate ventilation, elevated indoor humidity levels, and envelope moisture problems. An unbalanced ventilation system in these houses can lead to 
excessive house depressurization that in turn could cause combustion appliances to backdraft and spill combustion products indoors, or to increase the transport of pollutants into the house from adjacent unconditioned spaces (e.g., soil gases, vehicle exhaust gases).

How tight a house should or can be is a tradeoff between the cost of sealing and the cost of ventilating (naturally and/or mechanically), while maintaining acceptable indoor air quality. It is easier to make new houses tight than it is to seal older houses. However, building new houses with just the right leakage is a difficult task, because air leakage is strongly dependent on construction quality, even for houses with the same design. In the end, diagnostic testing is the only way to ensure that the desired envelope airtightness is achieved.

\section{Applicable Metrics}

The airtightness of the building envelope elements, both at the component level and together as a system, is a generic metric. We do not recommend the use of component level metrics for commissioning, because of the time and expense associated with diagnostic testing to determine them.

Specific metrics describe the airflow or air change rate at a standard pressure differential (e.g., $\mathrm{CFM}_{50}, \mathrm{CFM}_{25}, \mathrm{ACH}_{50}$ ), or effective leakage area (e.g., ELA 4 ). In some cases, intermediate parameters in equations used to calculate these metrics are used instead. These include terms such as the airflow coefficient and pressure exponent. In rare cases, economic factors are included with the airtightness metrics (e.g., $\$ / \mathrm{CFM}_{50}$ ).

Floor area and/or building height are sometimes used to normalize airtightness metrics to allow comparison between buildings or to Standards. An example is the specific leakage area (SLA) metric in Title 24, which represents the effective leakage area normalized by conditioned floor area. Another example is the normalized leakage (NL) in ASHRAE Standard 119 (1994), which represents effective leakage area normalized by conditioned floor area and by eave height relative to that of a one-story house.

For energy purposes, NL is a perhaps the most convenient metric because it approximately represents the seasonal time-weighted average air change rate due to natural infiltration alone. For an airtight one-story house, a normalized leakage of 0.1 is approximately equivalent to 2.1 $\mathrm{ACH}_{50}$ or an effective leakage area of $1.4 \mathrm{in}^{2} / 100 \mathrm{ft}^{2}(\mathrm{SLA}=1.0)$. An NL of 0.1 for a two-story house is approximately equivalent to $1.7 \mathrm{ACH}_{50}$ or an effective leakage area of $1.2 \mathrm{in}^{2} / 100 \mathrm{ft}^{2}$ $(\mathrm{SLA}=0.8)$. Using the same NL regardless of house height ensures that different height houses are on a level playing field in terms of energy consumption associated with infiltration. It also means that taller houses need to be more airtight. In contrast, the SLA metric allows taller houses to have greater natural infiltration and to use more energy compared to a one-story house.

An NL of 1.0 corresponds to a reference climate of about $3,500^{\circ} \mathrm{F}$-infiltration degree-days (IDD) with a seasonal infiltration energy load per unit floor area of $13 \mathrm{kBtu} / \mathrm{ft}^{2}$. NL scales inversely with IDD. As a result, a house in a climate with double the IDD needs to have half the NL to achieve the same infiltration energy load.

Because the normalized leakage metric in ASHRAE Standard 119 has more intrinsic meaning than any other metric, we recommend that it be the primary metric for commissioning all types of houses. 
A simple set of metrics related to airtightness includes air barrier type and location. It is easy to evaluate these two metrics using visual inspection during construction, but this is difficult afterwards.

\section{Recommended Diagnostics}

- Blower Door: Only one diagnostic tool is practical for commissioning the airtightness of the building envelope: a blower door. It consists of a calibrated flow meter combined with a fan mounted in a fabric or rigid panel, which is located in an open door or window. The device determines airflow through the envelope as a function of pressure differences imposed by the blower (ASTM 1997, ASTM 1999a, CGSB 1986). Including pressure gauges, it typically costs about $\$ 1,600$ to $\$ 3,500$.

Procedurally, a two-point blower door test with multiple pressure difference and flow readings at each point provides a statistically better envelope leakage estimate than multiple points with a single reading at each point (Sherman and Palmiter 1994, ASTM 1997). However, a two-point test cannot distinguish leakage differences due to envelope changes at different pressures (e.g., a vent damper opening as the pressure differential increases). Multiple points (greater than two) can more clearly show such compliance, as well as instrumentation failures. Single point tests, although quick, are unreliable for quantifying leakage, because there is no method to check the accuracy of the result. However, singlepoint tests are useful qualitatively during air-sealing work to quickly assess progress toward a planned air-tightness goal.

Blower door tests are susceptible to wind effects (Modera and Wilson 1990) and stack effects. A practical wind speed limit with a single outdoor pressure tap located in a sheltered region is approximately $8 \mathrm{mph}(13 \mathrm{~km} / \mathrm{h})$ or less. This constraint limits times for accurate testing. Manifolds connecting pressure taps on four faces of a building can be used in some cases for linear averaging and fluctuation damping of pressure-differences in an attempt to reduce wind-effect-related precision errors and to extend conditions under which the test may be carried out (CGSB 1986). General guidelines suggest testing only when the outdoor temperatures are in the range of 41 to $95^{\circ} \mathrm{F}$. In cold climates, this constraint severely limits times for accurate testing.

Blower doors used together with commercially-available smoke tubes help identify envelope leak locations and increase the effectiveness of envelope sealing efforts (ASTM 1995). This technique shows the location of leak entries and exits indoors or outdoors, but not the leakage paths themselves within the envelope assemblies. Smoke released near a leak is sucked into leak entries and is diffused at leak exits. This technique is inexpensive and rapid, but knowledge of likely leak locations is required to minimize the search effort. Searching for leaks on the building exterior can be especially difficult or impossible due to wind effects. The acidic nature of the smoke can cause material damage or occupant irritation if used in large quantities. Smoke tubes cost about $\$ 30$ to $\$ 50$ per tube.

To supplement the smoke tube testing, comparisons of thermographic scan images before and after pressurizing or depressurizing the building using a blower door can highlight air leakage paths that might otherwise be confused as insulation anomalies. Sufficient time between these scans must elapse for the leaks to equilibrate to the temperature of the air passing through them. The time depends on the airflow rate through the cracks, the temperature differential, and the envelope thermal mass. Although this technique is rapid, it 
is expensive and subject to the limitations described in the section about using infrared thermography for inspecting opaque building envelope assemblies. Consequently, this technique alone is not a reliable means to locate leaks; instead, smoke tube tests are required to confirm leaks located using this technique.

\section{Procedural References}

1. ASTM. 1999a. "ASTM Standard E779-99, Standard Test Method for Determining Air Leakage Rate by Fan Pressurization”. Philadelphia, PA; American Society for Testing and Materials. http://www.astm.org.

2. ASTM. 1997. “ASTM Standard E1827-96, Standard Test Methods for Determining Airtightness of Buildings Using an Orifice Blower Door". Philadelphia, PA; American Society for Testing and Materials. http://www.astm.org.

3. CGSB. 1986. "CAN/CGSB-149.10-M86, Determination of the Airtightness of Building Envelopes by the Fan Depressurization Method". Ottawa, CA; Canadian General Standards Board. http://www.pwgsc.gc.ca/cgsb/catalogue/specs/149/149 010-e.html.

4. ASTM. 1995. “ASTM Standard E1186-87, Standard Practices for Air Leakage Site Detection in Building Envelopes". Philadelphia, PA; American Society for Testing and Materials. http://www.astm.org.

\section{Moisture}

\section{Introduction}

Moisture problems are becoming increasingly prevalent throughout the country due to changes in the way buildings are constructed. Increasing energy efficiency and changes in the markets have driven construction practices toward designs that are much more susceptible to moisture problems. Even in California, whose dry climate normally exempts it from moisture concerns, the growth of moisture-related problems is troubling for builders, consumers, and policy-makers.

Various studies have attributed poor durability, material degradation, odors, and unhealthy indoor environments to these moisture problems. Poor durability can lead to increased maintenance costs (e.g., repairing peeling paint or wallpaper, removing efflorescence). Moisture in building components can produce a "self-composting house" in which wood rot and fastener corrosion eventually causes structural failure (Halvorsen et al. 2000). Loss of structural integrity is particularly troublesome in California, because the ability to withstand seismic loading may be impaired. While odors may only be unpleasant, they can indicate the presence of toxigenic molds. Microbiological growth adjacent to infiltration paths due to moisture within the building envelope can contaminate the indoor air and increase health problems. Snell et al. (2000) have linked asthma to mold growth supported by increased moisture levels in the indoor air and on surrounding surfaces. The American Lung Association (1999) now lists asthma as the leading serious chronic illness of children in the U.S.

\section{Applicable Metrics}

There are several metrics for moisture damage, but the literature that we reviewed does not commonly refer to them. Simple qualitative metrics include visible wetness or degradation of interior or exterior finishes and structural components. Degradation can include staining, streaking, bacterial or fungal growth, and wood rot. All these metrics are useful for 
commissioning existing houses; however, other than visible wetness, they are not relevant for new construction.

More complex and quantitative terms used by researchers involved with this issue and moisturedamage susceptibility include vapor partial pressure, condensation potential, mass of condensed water, surface water activity, water intrusion rate, diffusion path length, drying potential, and moisture content. The latter metric is the one most easily quantified during a field test of a new or existing house. At this time, we recommend that commissioning exclude the other quantitative metrics listed here, because of the difficulty in determining them.

\section{$\underline{\text { Recommended Diagnostics }}$}

Diagnostics to assess moisture damage are poorly developed. In particular, most diagnostics can only evaluate the presence of moisture, rather then the susceptibility to moisture damage. The recommended diagnostics involve visual and electrical inspection techniques.

In some cases, researchers have used conventional or pulsed infrared thermography to carry out rapid, non-destructive, broad-area scans with the intent of locating moist areas. However, these infrared methods are still in development; require expensive cameras, high-power flashes, sophisticated analyses, and highly trained personnel; and are useful only under limited conditions. As such, infrared techniques for moisture inspection are not ready for use in residential commissioning.

- Visual Inspection: A visual surface inspection for excess moisture within the building envelope can be carried out by determining the presence or absence of deficiencies such as wetness, microbiological growth (e.g., mold and mildew), discoloration, texture changes, material dimensional changes, decay, or structural dislocation. Surface inspections are a useful screening technique, but the absence of visible deficiencies does not exclude the presence of excess moisture within the envelope. As a result, invasive tests may be necessary when occupants have associated allergic symptoms and/or there is high relative humidity indoors with no significant internal moisture sources and proper ventilation. Invasive tests can include tactile probing of envelope sections with a sharp probe and dismantling of envelope sections for internal inspection. Internal inspection involves looking for similar problems as those listed for surface inspections, as well as for problems such as leaks from plumbing systems.

Visual inspection focuses on observing existing problems. It is not appropriate in general for use during construction, except for evaluating envelope detailing and plumbing system integrity.

- Electrical Inspection (Impedance Scanning \& Conductance Probing): Two types of electrical devices are well-developed and commercially available for field-assessments of the moisture content in building materials: surface scanning dielectric meters and penetrating conductance meters. The dielectric devices cost about $\$ 300$ to $\$ 6,000$; the conductance devices cost about $\$ 150$ to $\$ 900$.

Surface scanning devices emit low-frequency electromagnetic waves and detect their disturbance to determine average moisture content. These devices are believed to be good indicators of high relative moisture content near the surface of non-conductive porous building materials (wood, drywall, plaster, roofing, insulation, carpet, and concrete). As a result, they are best used for comparative sampling over different regions of a surface to 
indicate the range of moisture content (e.g., a suspected wet region to a known dry region of similar material). Apparent high moisture content regions can then be checked with a subsurface conductance probe. An advantage of this device is that it does not damage envelope surfaces. A further advantage is that it can rapidly test large regions and provide continuous readings. These devices are contact pressure sensitive (Margotta et al. 1984).

Penetrating conductance devices determine the moisture content of non-conductive porous building materials near their exposed surfaces. This method measures the resistance between the probes and correlates the resistance to moisture content. A disadvantage of this method is that it damages the envelope surface during probe insertion, which means that surfaces often require repair after such tests, even when there are no moisture problems. It is also time consuming to insert and remove probes at each test site. However, probe insertion has the advantage that it can also provide a tactile indicator of subsurface structural decay.

Although several studies describe the accuracy of these devices for use with wood products (James 1988, Warren 1994, ASTM 1998), there appear to be no published data describing their accuracy for other materials, especially when combined as building assemblies. For wood, ASTM (1998) describes conductance meter accuracy of about 7\% relative error (about \pm 0.5 to $2 \%$ moisture content); dielectric meter accuracy is about $\pm 5 \%$ moisture content. Warren (1994) notes that dielectric meters improperly weight moisture content for materials more than about $3 \mathrm{~mm}(0.125 \mathrm{inch})$ thick and are sensitive to undesirable field penetration beyond the target zone.

Simpson (1999) indicates that accuracy decreases when wood fibers are saturated (25 to $30 \%$ moisture content). Above $30 \%$, readings are at best relative comparisons of whether the sampled material is gaining or losing moisture. These devices are susceptible to static electricity and related errors at low (less than $8 \%$ ) moisture content. Conductive materials such as metal fasteners, flashing, and joints can cause falsely elevated moisture content readings.

\section{Procedural References}

To evaluate moisture-damage susceptibility of new construction, one can refer to best practices guides and lists of likely defects that may lead to future damage:

1. Lstiburek, J. and J. Carmody. 1994. "Moisture Control Handbook: Principles and Practices for Residential and Small Commercial Buildings". New York: Van Nostrand Reinhold. http://www.buildingscience.com/resources/books/default.htm.

2. RDH. 2000. "Building Envelope Rehabilitation: Consultant Guide". Report of RDH Building Engineering Limited to Canada Mortgage and Housing Corporation. Ottawa, ON: Canadian Housing Information Centre. April. http://www.cmhc-schl.gc.ca/publications/en/rh-pr/tech/2000-115.htm.

There are no formal standards for assessing water damage or measuring the moisture content in building assemblies. Two inspection agencies have recently developed diagnostic protocols for use in existing houses in an attempt to fill this void (NHCID 1998, GAHI 2000). These protocols address water damage that has occurred in southeastern US houses clad with Exterior Insulation and Finish Systems (EIFS).

1. NHCID. 1998. "Moisture Testing Guide for Wood Frame Construction Clad with Exterior Insulation and Finish Systems (EIFS), Version 3.01". Prepared for EIFS Review Committee. 
Wilmington, NC: New Hanover County Inspection Department. August 4. http://www.toolbase.org/Docs/MainNav/MoistureandLeaks/876 protocol5A.pdf.

The NHCID (New Hanover County Inspection Department) protocol is the most developed one available. It provides guidelines for locating and assessing moisture related damage in wood frame structures clad with drainable EIFS. It describes the use of a dielectric moisture meter for qualitative investigation, followed by subsequent quantitative assessment of suspected wet areas with a conductance meter. It also describes locations for wall scanning and probing, including below windows, doors, flashing, penetrations, and joints. In addition, it provides a comprehensive reporting format to document measurements. This protocol contains no precision and bias specifications, but it does suggest a field method for "calibrating" a dielectric meter.

2. GAHI. 2000. "The GAHI Protocol for Exterior Insulation Finishing Systems (EIFS) Moisture Intrusion Inspections - One \& Two Family Homes”. Atlanta, GA: Georgia Association of Home Inspectors. November 1. http://www.gahi.com/EfisProt.htm.

The GAHI (Georgia Association of Home Inspectors) protocol provides brief guidelines and recommendations for visually inspecting and testing houses clad with EIFS. The visual inspections focus on observing exterior water damage, as well as assessing exterior water drainage and intrusion management systems. It recommends that visual observations suggesting water entry be followed by moisture testing using a dielectric meter and then a conductance meter. It suggests conducting destructive structural investigations if moisture contents greater than $30 \%$ are measured. A related document (EIMA 2000) is largely based on the GAHI protocol. Its visual inspections focus on observing interior water damage, as well as assessing exterior water drainage and intrusion management systems. The EIMA (EIFS Industry Members Association) protocol requires only a conductance meter. It suggests conducting destructive structural investigations beginning at a lower threshold: $20 \%$. The EIMA protocol also provides a cursory form as guidance for recording inspection data. 


\section{Air Distribution Systems}

Residential air distribution systems include fans and ducts for space conditioning and ventilation. Poor construction and operation of the air distribution systems can cause comfort problems, poor indoor air quality, and structural moisture problems, as well as wasted energy. In particular, ducts that are part of the thermal distribution system may be the single worst performer in the energy performance of a house (Jump et al. 1996). Much of the problem is due to installing ducts outside conditioned space, duct leakage, duct insulation compression, and other poor installation practices.

Compared to the space conditioning system, the ventilation system in most houses is simple. It consists of operable windows, infiltration, and a few (if any) intermittently-operated local exhaust fans. However, such systems are not always reliable for their intended purposes. To address this issue, more houses are also beginning to use whole-house ventilation. For some of these houses, this function is an integral part of the space conditioning system operation. Furthermore, the proposed ASHRAE Standard 62.2 (2002) requires mechanical ventilation in all new houses. Such ventilation practices and requirements mean that minimizing the impact of airhandler and ventilation airflows on fan power and thermal loads is of increasing importance to utilities and energy planners (in terms of peak power) and residents (higher energy bills). At the same time, ensuring good delivery effectiveness and room-by-room distribution efficiency of thermal and ventilation distribution systems depends on maintaining proper airflow through these systems.

The simplest and most practical commissioning of air distribution systems involves assessing duct leakage, airflow through the air handler, and duct airflows.

\section{Duct Leakage}

\section{Introduction}

The intent of a residential thermal distribution system is to supply heating and cooling throughout the house. Of the 12 million residences in California, about 8 million have some sort of forced air heating and/or cooling system (Walker 2001). These systems use fans to circulate indoor air through sheet metal or flexible plastic ducts located in attics, crawlspaces, and garages, which are usually outside the heated and cooled parts of the house. Metal ducts are more common in older houses; almost all new houses use plastic ducts. Existing duct systems typically are only 50 to $75 \%$ efficient, depending on the climate, the specific location of these ducts, their leakage, and their insulation levels.

When the system is operating, air leaks in and out of ducts at all the connections within the system. These leaks occur at connections between the furnace/air conditioner and the duct system, at branches in the duct system, and at connections from the ducts to grilles. One of the greatest leakage problems occurs when there simply is not any duct: cavities in the walls or floors of the house are used as the "duct system".

One effect of duct leakage is that air that the occupants have paid to heat or cool escapes from the system before it can actually heat or cool the house. Another effect is that air leaking into the ducts increases the amount of outside air that needs heating or cooling. To understand this latter effect, consider that outside air is usually cooler (for heating) or warmer and more humid (for cooling) than air inside the house. As a result, the heating or cooling capacity of the system is then used to heat or cool this outside air instead of the air in the house. In particular, attics are 
perhaps the worst place to put the ducts, because sun shining on the roof during summer can heat these spaces to temperatures over $150^{\circ} \mathrm{F}$. This in turn heats up the ducts and increases the temperature of air entering the house through the supply registers, which decreases the ability of the air conditioning system to cool the house. In particularly poor systems, the air supplied to the house through the registers can be hotter than the air in the house and the cooling system ends up heating the house rather than cooling it. Even with the heating and cooling system off, the leaks in the ducts increase the ventilation rate of the house, which increases the need for heating or cooling.

\section{Applicable Metrics}

There are numerous metrics related to duct leakage and the thermal performance of residential air distribution systems, most of which have been developed over the past ten years.

Duct leakage metrics include duct air leakage flows (e.g., $\mathrm{CFM}_{25}, \mathrm{CFM}_{50}$ ), duct leakage class, and effective duct leakage area (e.g., ELA $4, \mathrm{ELA}_{25}$ ). These metrics can be subdivided into leakage to indoors and outdoors, as well as into return, supply, cabinet, and boot components. Related metrics include airflow through the air handler, airflow and pressure drop within a duct, power delivered to the duct system, power lost from supply ducts due to conduction and leakage, and fractional conduction loss (Walker et al. 1996).

Duct air leakage flows can be normalized by the airflow through the air-handler or by the conditioned floor area of the house. Title 24 explicitly uses the airflow rather than floor area to normalize duct leakage. The floor area is implicitly included in this metric because it assumes a nominal air-handler airflow rate of $400 \mathrm{cfm} /$ ton of cooling and these systems are often sized based on floor area per ton.

For commissioning existing houses, we recommend that duct air leakage flows to outside (separated into supply and return leakage at operating pressures) be used as the primary metric, normalized by the actual airflow through the air-handler. For assessing energy and indoor air quality issues, especially in existing houses with leaky systems, it is a more robust metric than duct leakage area, which requires supplementary knowledge of pressure differentials at each leak to describe flows. It is practically impossible to know those pressures. For commissioning houses with tight duct systems, either metric can be used, because the leakage flows are small and the uncertainty in defining leak pressure differentials is less important.

Metrics that are more complicated include thermal regain, delivery effectiveness, and distribution system efficiency (ASHRAE 1999). Each of these can be defined either on a design condition basis or on a seasonal basis. Thermal regain represents the fraction of heat transferred unintentionally into or out of a duct that returns to the conditioned space. Delivery effectiveness is the thermal energy transferred to or from the conditioned space divided by the thermal energy transferred at the equipment-distribution system heat exchanger. Distribution system efficiency is the energy actually consumed by the equipment connected to the distribution system divided by the energy that the equipment would consume if the distribution system was adiabatic, had no impact on equipment operation, and did not influence building loads. Obtaining all the necessary information in a house to evaluate these metrics is impractical during commissioning. However, as Title 24 already does, several assumptions can be made about house characteristics (e.g., duct surface area) to make evaluating these metrics practical when commissioning a house. 


\section{Recommended Diagnostics}

Several quantitative diagnostics are available to uniquely determine duct leakage (Walker et al. 1998a, Walker et al. 1999, Francisco and Palmiter 2000): the duct pressurization test, the DeltaQ test, the house pressure test, and the nulling pressure test. The pressure pan test (Siegel and Manclark 1998) is not included in this list, because its pressure indication does not uniquely determine leakage. As a result, we do not recommend it for commissioning except as a rapid screening supplement to more elaborate tests.

Researchers are in the process of improving the cost and accuracy of duct leakage testing. They are also rewriting the ASTM Standard E1554 (1994) for measuring duct leakage to include newer methods. We expect its revision will be complete in 2003. The standard (and our discussion) focuses on the existing duct pressurization test and the new DeltaQ test, because they are the simplest in terms of equipment and procedure, are more robust, and in some cases are more flexible in terms of the types of houses or systems that can be tested.

- DeltaQ Test: This new test is based on changing the airflows through distribution system leaks by turning the air handler on and off while pressurizing and then depressurizing the building envelope to various pressure differentials using a blower door. The test gets its name from the difference in flows (the common symbol for flows being Q), hence the name DeltaQ. A unique feature of this test (compared to duct pressurization) is that it determines envelope leakage at the same time as duct leakage.

Air leakage to outside through supply and return leaks is determined directly with the ducts at operating pressures. Testing the system at operating pressure significantly reduces the uncertainty that occurs when converting measured duct leakage at other than operating pressures to measured operating pressures. The DeltaQ test can only be used in finished houses, because it requires an envelope that can be pressurized.

A substantial advantage of the DeltaQ test is that it is cheaper than other duct leakage tests. It uses equipment (a blower door) that is widely available and that almost all people interested in duct leakage already have (e.g., weatherization program participants). This reduces the capital cost to carry out the tests. In addition, there are no requirements for grille sealing or for separation of the supply and return systems at the air handler by a seal. Compared to the duct pressurization test, the DeltaQ test is less time-consuming (lower labor costs) and is less susceptible to leakage caused by poor sealing or seal failure during the test. However, there can be a tradeoff between increased capital costs for automating the test and the time savings resulting from the automation. Together, data acquisition systems and computers to record the data cost about $\$ 3,200$ to $\$ 4,500$. However, the time saving, record keeping ability, and operator error reductions resulting from automating the test outweigh the higher capital cost of this option. Without automation, pressure gauges to carry out this test cost about $\$ 450$ to $\$ 750$.

Test precision and bias are susceptible to wind speed and direction variation that result in envelope pressure fluctuations. Resulting fractional errors in duct leakage can be large, although absolute flow errors will remain small. The practical limit for wind speeds when accurate testing can be carried out is not currently known; research to determine the wind limitations for this test is in progress. Preliminary data for a single building indicate good repeatability: 0.5 to $1.0 \%$ of air-handler airflow with a $95 \%$ confidence interval (Walker et al. 2001). These data are from 20 tests in mild weather for wind speeds of $5 \mathrm{mph}(8 \mathrm{~km} / \mathrm{h})$ or 
less with a tight duct system and envelope (about 7\% return leakage, $2 \%$ supply leakage, and a normalized envelope leakage of about 0.5 ).

This test is also susceptible to stack effects, which may affect the apparent leakage area of the envelope and duct system. However, this bias is not expected to be large. Outdoor temperature limits to reduce stack-effect-induced pressure variation are unknown, but we expect that the suggested outdoor temperature limitations for envelope airtightness tests are probably also applicable.

- Duct Pressurization Test: This test is the most common one performed on duct systems. It is analogous to airtightness testing of building envelopes in that the test measures airflows at specified pressure differences. All the supply and return grilles in a system are covered and a measured amount of air is blown into the ducts through a small fan-assisted flow meter. Resulting flows and duct pressures indicate how leaky the ducts are. However, the test only measures the combined size of holes in the duct system, and not the flow through them to outside at operating conditions (duct pressures are approximately uniform during this test, whereas operating duct pressures are not uniform). The test device is expensive: typical costs are about $\$ 1,500$ to $\$ 2,000$.

To translate from hole size to airflow, these tests require that a duct system operating pressure be assumed (often based on measured plenum and/or register pressures). An advantage of measuring these pressures is that they are also a useful indicator of ducts that are undersized or that are restrictive due to their topology and fittings.

The conversion from hole size to airflow has large uncertainties for leaky duct systems, because the assumed pressure may be a poor indicator of the pressures at duct leaks, whose location is unknown. However, for verifying that ducts have little leakage, a pressurization test is useful because even large errors in estimating the pressure difference across small holes will not result in large leakage flow uncertainties. This is one reason why many utility and weatherization programs use this test as a screening tool and why codes and standards are gradually adopting it. Other reasons include:

- Robustness. The duct pressurization test has almost no restrictions on the type of system it can be used on, or the weather conditions during the test. In particular, it can be used in unfinished or finished houses. This test is susceptible to wind and stack effects, but less so than for the DeltaQ test, because it does not use envelope pressure in its calculations to determine duct leakage.

- Repeatability. The combined results of several research projects (Walker et al. 1998a, 1998b) together with the field experience of other users have shown that the repeatability of duct pressurization testing is very good.

- Simplicity. It is easy to interpret the results of a duct pressurization test without having to perform many (or any - with the appropriate hardware) calculations. This allows the test crew to evaluate the ducts during the test and to immediately assess the validity of the results.

- Familiarity. Work crews that have performed envelope leakage tests are familiar with the test method for ducts, because envelope testing uses a similar apparatus and calculation methods. 
There are several variations of this duct leakage test, which involve increasing time and equipment requirements (and complexity):

- Total Leakage. This is the simplest and most often used test. Supply and return ducts are tested together, so the split between supply and return leaks must be guessed. In addition, the fraction of total leakage that leaks to outside for energy losses also must be guessed.

- Leakage to outside. For these tests, another fan is used to pressurize the house to the same pressure as the ducts, so that any remaining duct leakage flows are only to outside. This test has the additional complication of requiring two fans and extra pressure measurements. In addition, it requires more time because the pressures across the ducts and the building envelope must be balanced.

- Supply/return split. To separate supply and return ducts, a physical barrier must be installed, usually inside the air handler cabinet or return plenum. The two sides of the duct system can then be tested separately to determine the supply/return split.

Because the pressurization test is usually implemented to measure the total leakage of the ducts and not just the leakage to outside, it will overestimate the leakage required for energy loss estimates. However, from a code compliance testing point of view, this error is in the right direction because it means that the true losses will be less than those indicated by the test. In other words, a system whose total leakage passes a leakage specification is guaranteed to have leakage to outside that is less than the specification.

For energy ratings of homes with leaky ducts (e.g., assessing distribution system efficiency using ASHRAE Standard 152P or Title 24 methods), the simple pressurization test can be inappropriate due to the assumptions about pressures across the duct leaks (Walker et al. 1998b, Francisco and Palmiter 1999). In these cases, other methods such as the DeltaQ test may give better results. Leaky ducts tend to produce a larger pressure signal for the DeltaQ test, which reduces its uncertainty.

One of the biggest drawbacks to the pressurization test is the need to cover all the grilles and attach the flow and pressurization equipment. Sealing grilles can be difficult (e.g., hard to reach on high vaulted ceilings) and time consuming (e.g., taping each grille, often while standing on a ladder). In addition, detailed versions of the test require inserting a blockage to separate the supply and return and using a blower door to match the duct and house pressures - both of which can be time consuming. Sometimes, it is also difficult or impossible to install the air-handler seal due to the equipment configuration. All the grille and air-handler seals are susceptible to failure during the test. If any of the seals fail, the test will overestimate the duct leakage because it will include the seal leakage.

\section{Procedural References}

1. ASTM. 1994. "ASTM Standard E1554-94, Standard Test Methods for Determining External Air Leakage of Air Distribution Systems by Fan Pressurization". Philadelphia, PA; American Society for Testing and Materials. http://www.astm.org.

2. ASHRAE. 1999. "BSR/ASHRAE Draft Standard 152P-1999, Method of Test for Determining the Design and Seasonal Efficiencies of Residential Thermal Distribution Systems". Atlanta, GA; American Society of Heating, Refrigerating, and Air-Conditioning Engineers, Inc. 


\section{Air-Handler Airflow}

\section{Introduction}

ACCA Manual S (1995) describes a design procedure for estimating the air-handler airflow required for a residential cooling system at design conditions. It results in flows of 415 to 545 $\mathrm{cfm} /$ ton of total evaporator capacity (sum of sensible and latent capacities). The lower airflows correspond to high latent loads (humid climates) and result in a colder evaporator and more moisture removal than occur with higher airflows. These flows are intended to minimize fan power and achieve adequate humidity control while avoiding evaporator icing. Manufacturers typically recommend a lower airflow range: 350 to $450 \mathrm{cfm}$ per nominal ton of evaporator total capacity. The airflow target in the AB970 2001 revisions to Title 24 is in the lower half of the manufacturer range: $385 \mathrm{cfm} /$ ton (Wilcox et al. 2001).

Often, installed equipment does not meet any of the targets described above. For example, Blasnik et al. (1996) tested 28 systems and found that more than 50\% had flows below 350 $\mathrm{cfm} /$ ton. The average system had $86 \%$ of the manufacturer's recommended airflow (standard deviation of $16 \%$ ). The worst system had as little as $57 \%$ of the manufacturer's recommended airflow. Seven systems had flows that were at or above the manufacturers recommended flow. A common reason for low airflow was an undersized, restrictive flex duct system.

Manufacturers recommend corrective action when airflows are below $350 \mathrm{cfm} /$ ton, because airflows this low can cause evaporator icing. Ice on the evaporator insulates it and increases system flow resistance, which further reduces air-handler airflow. The resulting reduction in heat transfer from the air to the refrigerant can cause liquid refrigerant to enter the compressor and damage it.

Too low an airflow also reduces total and sensible cooling capacity, and reduces efficiency. However, latent capacity increases as airflow decreases (ACCA 1995). This occurs because sensible capacity decreases faster than total capacity. Rodriguez (1995) tested the effect of reduced airflow on two 3.5-ton heat pumps. One heat pump had a reciprocating compressor and a short-tube-orifice expansion device; the other had a scroll compressor and a TXV. For the short-tube-orifice-controlled heat pump, these tests indicate that a $14 \%$ reduction in flow (e.g., to $344 \mathrm{cfm} /$ ton from $400 \mathrm{cfm} /$ ton) reduces total capacity by $7 \%$ and energy efficiency ratio (EER) by $6 \%$. For the TXV-controlled heat pump, the reductions in capacity and EER are less (only about $2 \%$ at the same airflow reduction).

\section{Applicable Metrics}

In the simplest form, metrics for air-handler airflow are in flow units when designing a house. Metrics that are slightly more complex are normalized by floor area (e.g., $\mathrm{cfm} / \mathrm{ft}^{2}$ when discussing ventilation) or by total cooling capacity (e.g., cfm/ton when discussing thermal loads) to allow system-to-system or house-to-house comparisons. When commissioning a house, we recommend that the unnormalized metric be used alone (e.g., cfm at operating conditions by itself), because the total flow affects both thermal loads and ventilation.

\section{Recommended Diagnostics}

Several diagnostic tools and techniques can be used to determine airflow through the air-moving equipment of cooling and heating systems. Two of the most promising practical and commercially available tools include a fan-assisted flow meter (Walker et al. 1999) and a flow plate and grid (Palmiter and Francisco 2000). The sum of individual grille airflows measured 
with a fan-assisted flow hood can also be used to assess air-handler airflow through tight duct systems, but this method requires accurate duct leakage measurements to confirm that the ducts are indeed tight.

There are other techniques to assess air-handler airflow, but we do not recommend using them. They include using:

- the air temperature difference across the air-handler (Downey and Proctor 1999),

- a tracer gas (Walker et al. 1998b), or

- fan curve interpolation.

Of these other techniques, the first is prone to errors of more than $20 \%$ in many cases and nearly a factor of two in worst cases, due to flow nonuniformities and radiant effects (Palmiter and Francisco 2000). It is particularly problematic when used to assess airflows in systems that have inadequate refrigerant charge, because of the wide variation in air temperatures downstream of the evaporator coil (Wray et al. 2002).

While using a tracer gas can be very accurate, it requires expensive, delicate equipment and a well-trained technician. It is also subject to flow-nonuniformity-induced errors, which are difficult to assess in the field.

Fan curve interpolation cannot be relied upon, because manufacturer's fan curve data are not easily or generally available for residential systems. When it is available, it may not accurately represent the installed performance of the fan due to the inlet and exit flow restrictions of the airhandler cabinet that are not included in manufacturer's data. These effects can vary significantly and are difficult to estimate. In addition, it is not possible to measure the static pressure drop in the duct system at the same locations as those used to produce the manufacturer's fan curve.

- Fan-Assisted Flow Meter: This device uses a calibrated flow meter combined with a fan to determine air-handler airflow. It can be attached to the air-handler cabinet at its access door, or at a return grille if the return ducting is well sealed. In either case, it is time-consuming to attach the flow measurement device. Additional time is required to seal off the return upstream of the air handler if the device is connected to the air-handler cabinet.

With the air handler running, air is blown into the air handler or return (with the return sealed if connecting to the air handler). The goal of this test is to reproduce the pressure difference between the supply plenum and conditioned space under normal system operating conditions. If this operating pressure cannot be achieved due to capacity limits of the fan on the flow meter, then multiple measurements of flow and supply plenum static pressure are used to extrapolate to the operating pressure. The measurement device is essentially the same as the one used in duct pressurization tests (cost is about $\$ 1,500$ to $\$ 2,000$ ).

The accuracy of this method is not well defined at this time and cannot be easily estimated, due to the possibility of flow pattern changes within the air handler or static pressure measurement errors. However, based on field tests using tracer gas (Walker et al. 1998b), we expect the accuracy of the test method is approximately $\pm 10 \%$. Wray et al. 2002 have demonstrated that airflows obtained using this method in three houses agree very well with the measured sum of supply register flows and supply duct leakage (within 2 to 3\%). Agreement was poorer when compared to the sum of return flows and return duct leakage, 
but this may have been due to difficulties in measuring return flows. Further research is needed to establish the accuracy of this method.

- Flow Plate and Grid: This new device uses a calibrated multiple-orifice plate with attached upstream and downstream pressure manifolds to determine air-handler airflow. The device is inserted into an air filter body in place of the filter. Its pressure drop is intended to be similar to that of an air filter. In general, the "measured" airflow needs to be corrected to account for the difference in supply plenum static pressure with the device installed instead of the filter. In addition, when the flow plate is mounted in place of a grille filter, the return duct leakage needs to be added to the measured flow. The device costs about $\$ 1,100$.

Using fan-assisted flow meter measurements as a reference, preliminary comparisons of airflows obtained using the flow plate and grid in 74 houses indicate its measurements are within 17\% of the "reference" method (Palmiter and Francisco 2000). In 54\% of the houses, differences are less than 5\%. Reasons for differences are not reported, but might be related to plenum static pressure measurement errors. Francisco (2000) has indicated that upstream flow disturbances, such as $90^{\circ}$ bends in the return duct as close as 3 inches $(75 \mathrm{~mm})$ to the plate, do not adversely affect measurement accuracy.

Comparisons of field measurements in three houses by Wray et al. (2002) indicate a negative bias of 9 to $14 \%$ for airflows obtained using the flow plate and grid relative to fan-assisted flow meter measurements. The reason for the bias is unknown, but it is unlikely due to flow bypass because the rigid plate was taped to the air handler cabinet in each test.

- Sum of Grille Airflows: Measuring individual airflows at grilles, summing them, and accounting for duct leakage is a practical method to determine air-handler airflow, especially if these flows and the duct leakage are already being measured as part of whole-house commissioning. However, caution in diagnostic equipment selection is required. In particular, if grille airflow measurements are to be used to estimate air-handler airflow and a reasonable limit for deciding to change the equipment airflow is a $5 \%$ deviation from a specified norm, then the measurement needs to have an overall accuracy better than about $\pm 10 \%$. For tight duct systems (less than $6 \%$ duct leakage), a fan-assisted flow hood meets these requirements. Commercially-available flow hoods should not be used for this purpose because they are not accurate enough (typically $20 \%$ to $30 \%$ errors in measured flow, Wray et al. 2002).

\section{Procedural References}

There are no formal consensus standards for field measurement of air-handler airflow. As a result, commissioning must rely upon procedures defined by manufacturers of airflow diagnostic tools, subject to the accuracy constraints described above.

\section{Distribution System Airflows}

\section{Introduction}

For many years, the HVAC industry has measured grille airflows. However, until recently, almost all measurements were made in non-residential buildings, usually as part of a testing and balancing procedure for the HVAC system. Residential HVAC systems very rarely have been tested (usually by the research community). Now, utility programs, weatherization programs, and developing codes and standards (that require commissioning of residential HVAC systems) are beginning to consider addressing this important aspect of residential HVAC system performance. 
Measurements of individual grille flows can be used to identify parts of a system with large leaks (e.g., disconnected ducts), to determine flow imbalance between different spaces in a building, and, when combined with temperature and humidity measurements, to determine if enough heating or cooling is delivered by a grille to the space it serves. For identifying large leaks or disconnected ducts, the airflow changes are large and the accuracy of the measurement is not critical: being within $\pm 50 \%$ of the correct flow is reasonable. Similarly, imbalances in residential airflows between different spaces in a building are only critical at higher flow rates and an accuracy of $\pm 25 \%$ is probably reasonable.

The accuracy requirement related to providing enough heating or cooling airflow to a space for occupant comfort depends on how well the space, the rest of the building, and outside are thermally connected. For most typical residential rooms, there is little thermal resistance to other parts of the building; this reduces the sensitivity of room temperature to deviations in individual supply grille airflows. A simple steady-state heat balance for a room with an insulated exterior wall containing a window and uninsulated partitions for its other surfaces shows that a reasonable limit is to have supply grille airflows within $\pm 20 \%$ of their design specification to keep the room temperature within approximately $\pm 1^{\circ} \mathrm{C}\left(2^{\circ} \mathrm{F}\right)$ of the adjoining room temperatures. Therefore, the accuracy of the flow measurement device needs to be better than this $\pm 20 \%$ requirement. It is important to note that the sensitivity depends on building loads and therefore climate. The larger the load, the more precisely one needs to know the grille flow in order to maintain reasonable temperature limits. In addition, if transient performance is an issue (how fast a particular room is heated or cooled by the system), then the supply grille airflows need to be better defined.

\section{Applicable Metrics}

There are numerous airflow and ventilation-related metrics, which have been developed over about a 20 year period. Many can be used at component, room, or whole-house levels. They include metrics such as duct and ventilation airflows, air exchange rates, temporal and spatial ventilation effectiveness and efficiency, and indoor-outdoor and interzonal pressure differentials.

Some of these metrics can be subdivided. In particular, the temporal distribution of air within a room or entire house can be represented by metrics such as age of air, turnover time, and effective ventilation rate. Carbon dioxide $\left(\mathrm{CO}_{2}\right)$ levels indoors are sometimes used as a surrogate metric to quantify ventilation adequacy, but may be inappropriate when there are other pollutants of concern within the house. An additional metric useful to discussing infiltration-based ventilation airflow potential is infiltration degree-days (IDD). Parameters used in infiltration or ventilation simulation models also represent metrics that can be used to characterize how a house will perform in terms of ventilation. Such terms include surface pressure coefficients, as well as terrain and shielding parameters, all of which are related to wind effects.

When assessing time-varying airflows and ventilation in terms of controlling indoor air quality, the quantity of interest is often the temporal average rather than the peak. Apart from duct and ventilation airflows, and indoor-outdoor and interzonal pressure differentials, perhaps the most important airflow and ventilation-related metric that can be practically determined during residential commissioning is the effective ventilation rate (Sherman and Wilson 1986, Yuill 1986, Yuill 1991, ASHRAE 2001a).

The effective ventilation rate is the steady-state rate that would yield the same average contaminant concentration over the period of interest in the occupied space as does the actual 
sequence of time-varying discrete ventilation rates over the same period and in the same space. This effective rate is only equal to the simple arithmetic average rate when the discrete ventilation rates are constant over the period of interest and the contaminant concentration has reached its steady state value. Simple arithmetic averaging of instantaneous ventilation rates or concentrations cannot generally be used to determine these averages due to the non-linear response of indoor concentrations to the ventilation rate variations.

ASHRAE Standard 136 (ASHRAE 2001b) provides a set of factors to assist in calculating the annual effective air change rate of houses, for use in evaluating the impact of these air change rates on indoor air quality. Metrics such as envelope airtightness (in terms of normalized leakage area as defined by ASHRAE Standard 119) and ventilation system airflows need to be determined to use Standard 136.

Several metrics represent the performance of heat recovery devices in ventilation processes. These include terms such as sensible, latent, and total energy recovery effectiveness; sensible and total heat recovery efficiency; temperature ratio; ventilation reduction factor; and exhaustair-contamination ratio. Determining these metrics requires complex tests, which are not practical during residential commissioning.

\section{Recommended Diagnostics}

Several diagnostics are available to determine airflow rates through air distribution systems. They include: a fan-assisted flow hood (Walker et al. 1999), a conventional flow hood (no fan assist), anemometry, a pitot-static-tube traverse, or a flow grid. Of these, only a fan-assisted flow hood is consistently accurate and reliable. At this time, it is the only method we recommend for commissioning residential air distribution system airflows.

The other methods are error prone due to the effects of flow nonuniformities, difficulties in estimating effective flow areas (anemometry), uncertainty in determining insertion depth (pitotstatic tube), low velocities, misaligned sampling of the air stream, fouling (flow grid), and duct leakage. Of particular note, conventional non-powered flow hoods are sometimes an order of magnitude less accurate than fan-assisted flow hoods. Wray et al. (2002) have shown that measurement errors for these non-powered hoods are typically 20 to $30 \%$, but individual errors can be as large as $50 \%$.

A new simple $\$ 200$ device has recently become available to measure exhaust fan flows (Nelson 2001). It involves placing a gasketed box with a calibrated variable-sized orifice over the fan inlet and measuring the pressure drop across the orifice. The manufacturer reports that its accuracy is about $10 \%$ over a range of 10 to $120 \mathrm{cfm}$. However, it requires independent testing to assess its accuracy and usability.

- Fan-Assisted Flow Hood: A fan-assisted flow hood is similar to a conventional hood, but also has a fan-assisted flow meter attached by a flex duct. Unlike a conventional hood, this device does not require an elaborate seal at the gap between the hood and the adjacent building surface, because the integrated fan maintains a relatively neutral pressure difference between the interior and exterior of the hood. At the same time, the fan assist also eliminates backpressure problems that conventional flow hoods create. The location of the measurement device at the end of 6 feet $(2 \mathrm{~m})$ or more of flex duct attached to the flow hood virtually eliminates errors due to flow non-uniformities. 
Laboratory tests of a fan-assisted flow hood by Wray et al. (2002) indicate this device can achieve significantly better accuracy than conventional non-powered flow hoods: its RMS error is approximately $3 \%$ and no error is worse than $5 \%$. Unfortunately, such devices are not yet commercially available, but can be easily constructed of readily available components.

\section{Procedural References}

There are no formal standards for field measurement of air distribution airflows using fanassisted flow hoods. As a result, commissioning must rely upon procedures defined by manufacturers of related diagnostic tools, subject to the accuracy constraints described above.

For assessing effective ventilation rates, ASHRAE Standard 136 together with ASHRAE Standard 119 should be used.

1. ASHRAE. 2001b. “ANSI/ASHRAE Standard 136-1993 (RA 2001), A Method of Determining Air Change Rates in Detached Dwellings". Atlanta, GA; American Society of Heating, Refrigerating, and Air-Conditioning Engineers, Inc. http://www.ashrae.org/.

2. ASHRAE. 1994. “ANSI/ASHRAE Standard 119-1988 (RA 94), Air Leakage Performance for Detached Single-Family Residential Buildings". Atlanta, GA; American Society of Heating, Refrigerating, and Air-Conditioning Engineers, Inc. http://www.ashrae.org/. 


\section{Cooling Equipment}

\section{Refrigerant Charge}

\section{Introduction}

Residential central cooling systems typically are split systems, which have a condenser, fan, and compressor located outdoors adjacent to the house within a packaged condensing unit, and an evaporator and refrigerant control located indoors within the air-handler cabinet or an attached plenum. Two copper tubes containing refrigerant (i.e., the suction and liquid lines) penetrate the building envelope and connect these components.

When the cooling system works properly, the refrigerant flowing through the evaporator removes heat from the warmer indoor air blown across it. Evaporating the low-temperature, lowpressure refrigerant at constant temperature and pressure absorbs heat. The refrigerant leaves the evaporator as a superheated vapor and passes through the suction line into the compressor. In the compressor, the refrigerant temperature and pressure increase as the vapor is compressed. The hot vapor exiting the compressor enters the nearby condenser, where the cooler outdoor air blown across it removes heat. Condensing the high-temperature, high-pressure refrigerant at constant temperature and pressure rejects heat. The refrigerant leaves the condenser as a subcooled liquid and passes through the liquid line into the refrigerant control near the evaporator. This control acts as a flow restriction, which reduces the refrigerant pressure back to the evaporator pressure. The refrigerant temperature decreases back to the evaporator temperature as the pressure drops. As a result, low-temperature, low-pressure refrigerant enters the evaporator and the cycle repeats.

Even in new houses, cooling systems rarely perform as intended (Sherman et al. 1987). Aside from inadequate airflow across evaporator coils, refrigerant charge deficiency is a significant cause of this problem. Based on tests of more than 4000 residential cooling systems in California, it is clear that many systems have an incorrect refrigerant charge (Proctor 2000). Data from these tests indicate that about $34 \%$ are undercharged, about $28 \%$ are overcharged, and only about 38\% have correct charge. In the past, data from Blasnik et al. (1996) and Proctor (1997) have indicated that an undercharge of $15 \%$ is common.

Refrigerant charge has a particularly important impact on the capacity and efficiency of cooling equipment without a thermostatic expansion valve (TXV). For example, laboratory test data from Farzad and O'Neal (1988) for capillary-tube-controlled equipment indicate that a common charge deficiency of $15 \%$ can reduce equipment cooling capacity by 8 to $22 \%$ and the energy efficiency ratio (EER) by 4 to $16 \%$, depending on outdoor conditions.

\section{Applicable Metrics}

Because the amount of refrigerant in the cooling system has such an important impact on the performance of non-TXV-controlled equipment, the refrigerant mass ("charge") within the system is a metric. Coil volume and refrigerant line length are related metrics, because they affect the amount of refrigerant that a system requires.

Equipment manufacturers' refer to cooling equipment performance in terms of capacity and efficiency. Capacity represents the ability of the cooling equipment to remove heat.

Manufacturers often report it in units of tons $(12,000 \mathrm{Btu} / \mathrm{h})$ and usually refer to it in terms of the nameplate rating or the ARI rating. Sometimes, it is called the "installed" capacity. These metrics typically represent the capacity for a fixed set of indoor and outdoor operating 
conditions. A related metric is the required capacity that is determined using load calculations and that is used to size equipment. These capacities can represent the system as a whole or its components (e.g., evaporator, condensing unit).

Common metrics associated with the equipment efficiency are the energy-efficiency ratio (EER) and the seasonal energy-efficiency ratio (SEER). EER is an instantaneous measure that represents the total cooling capacity of the cooling equipment (Btu/h) divided by the total electrical input (Watts). The electrical input includes the power supplied to the condensing unit and to the air-handler fan. SEER is an integrated measure that represents the total cooling capacity of the cooling equipment $(\mathrm{Btu} / \mathrm{h}$ ) during its normal annual usage period divided by the total electrical input (Watt-hours) during the same period. It includes the effects of equipment cycling on capacity and power consumption. The term coefficient of performance (COP) is also a common metric that is applied to heat pumps more so than to air conditioners. It is defined in the same way as the EER, but it uses common units instead and is dimensionless.

To account for equipment, installation, and operation deficiencies, Neal (1998) has proposed the use of another metric: field adjusted SEER (SEERFA). This metric represents the seasonal performance of an installed cooling system (equipment and attached ducts). System performance degradation may occur due to improper refrigerant charge, inadequate evaporator airflow, duct leakage, and equipment oversizing. Neal also describes an approximate method for quantifying SEERFA. That method uses several correction factors to adjust the "rated" SEER of the cooling equipment and to extend it to encompass system performance. Neal bases the correction factors on limited data from his literature review. There has been no subsequent fieldwork to validate their use. Substantial research is required before Neal's metric and method can be broadly applied within the scope of residential commissioning. That work includes: developing a practical diagnostic that can determine absolute or fractional charge deviation; determining the variation of SEER with evaporator airflow; determining appropriate corrections to accommodate the range of airflow deviations, duct leakage, and oversizing found in the field; and determining how all the performance degradation effects should be combined.

"Tons at the register" (Walker et al. 1998b, 1998c) is a metric related to the combined thermal performance of residential air distribution systems and cooling equipment. It is a measure of enthalpy flow delivered intentionally at each supply register. Evaluating this metric requires measuring supply airflow, temperature, and humidity at each grille after the system conditions have reached steady-state. Currently, only one commercially available device is available to conduct such diagnostics. Our tests of a prototype of this device found significant airflow measurement problems, which the manufacturer is trying to resolve. As a result, this metric is currently not practical for commissioning, but will be as soon as appropriate diagnostic equipment is available.

Of these metrics, refrigerant charge is the most practical to determine during the course of residential commissioning. Apart from simply measuring total electricity consumption over a fixed period and "tons at the register", or visually checking equipment control settings (e.g., room thermostats, heat pump defrost and strip heat thermostats), evaluating the other metrics in the field requires complex and time-consuming diagnostics.

\section{Recommended Diagnostics}

Several diagnostic techniques are available to assess the amount of refrigerant charge in residential cooling equipment. These techniques include: superheat or subcooling tests, a 
gravimetric test, a sight glass, thermostatic expansion valve (TXV) frosting, "feeling the lines", and motor signature analysis. Of these, only the superheat and subcooling tests are quantitative, practical, well developed, and reliable. Those latter two tests are not standardized, but equipment manufacturers commonly specify them.

When a charging scale, refrigerant recovery unit, vacuum gauge, and vacuum pump are used (total cost of about $\$ 1,500$ to $\$ 2,000$ ), the gravimetric test can accurately determine the amount of refrigerant and non-condensable fluids in a cooling system by removing, weighing, and replacing them. However, this test is problematic because the amount of refrigerant required for optimal system performance is often unknown. This problem occurs due to the use of refrigerant line lengths and coils that do not conform to manufacturer's specifications. In particular, it is difficult to quickly estimate the internal volume of coils, especially when tubing wall thickness is unknown. Furthermore, volumetric calculations are insufficient to account for evaporator heat transfer characteristics that differ from those of the manufacturer-specified coil. Only a functional performance test such as the superheat or subcooling test can account for this difference.

Superheat and subcooling tests (Downey and Proctor 1999) assess charge adequacy by determining the refrigerant thermodynamic state at a specific point in the system. For the superheat test, which is used for cooling equipment with a fixed-orifice metering device (capillary-tube or short-tube orifice), the location is the compressor inlet at the condensing unit. The intent is to ensure that the refrigerant vapor is fully evaporated upon leaving the evaporator. For the subcooling test, which is used for equipment with a $T X V$ metering device, the location is the condenser exit at the condensing unit. In this case, the intent is to ensure that the refrigerant vapor is fully condensed upon leaving the condenser.

Superheat tests are more important than subcooling tests, because non-TXV-controlled equipment is substantially more sensitive to typical charge deficiencies. As such, we will discuss the superheat test here. We present more detail about this test than we do for other tests in this guide to help the reader understand the test complexity.

Superheat Test (Non-TXV equipped): In the superheat test, refrigerant suction line temperature and pressure, condenser inlet air dry-bulb temperature, and evaporator inlet air wet-bulb temperature are measured after the cooling equipment runs for about 15 minutes to achieve equilibrium (steady-state operation). "Measured" superheat is based on the difference between the measured suction line temperature and the refrigerant saturation temperature corresponding to the measured refrigerant pressure. Proper charge is typically indicated by a "measured" superheat not lower than $5^{\circ} \mathrm{F}\left(3^{\circ} \mathrm{C}\right)$ and within $5^{\circ} \mathrm{F}\left(3^{\circ} \mathrm{C}\right)$ of a target superheat at test conditions. Refrigerant charge is low if the "measured" superheat is more than $5^{\circ} \mathrm{F}\left(3^{\circ} \mathrm{C}\right)$ above the target superheat at test conditions. Charge is high if the "measured" superheat is more than $5^{\circ} \mathrm{F}\left(3^{\circ} \mathrm{C}\right)$ below the target superheat at test conditions.

The target superheat is determined using the equipment "superheat chart". Figure 1 (Downey and Proctor 1999) shows such a chart, which typically relates superheat $\left(5\right.$ to $40^{\circ} \mathrm{F}, 3$ to $\left.22^{\circ} \mathrm{C}\right)$ as a function of outdoor air dry-bulb and return-air wet-bulb temperatures. The intent of these targets is to facilitate equipment charge evaluation when it is operating at other than design conditions. Lower superheats are not listed, because they correspond to overcharging, which increases the risk of compressor flooding and associated damage. 


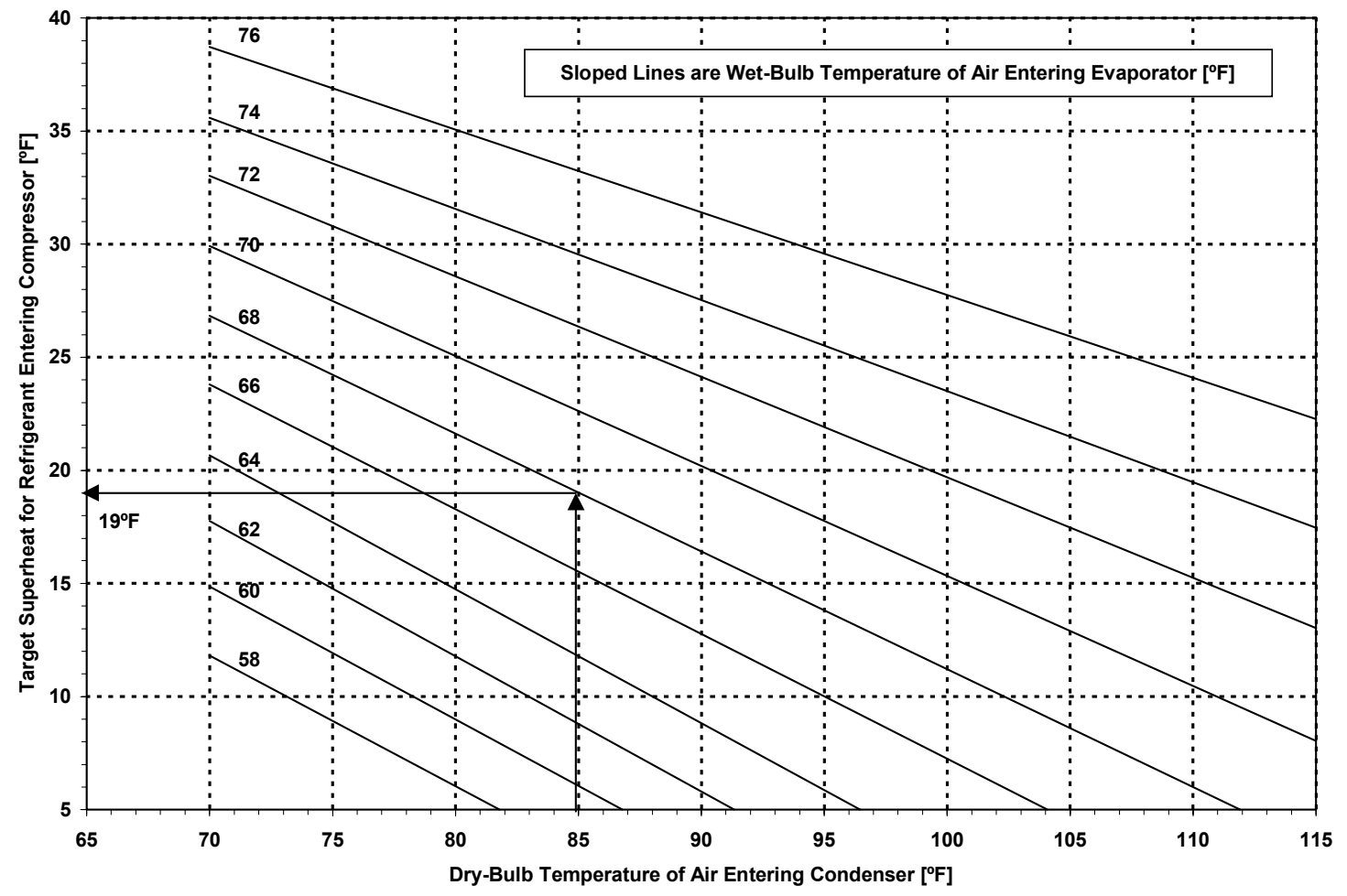

Figure 1. Sample refrigerant superheat charging chart.

The superheat test cannot be used in hot, dry climates, when there is a low return-air wetbulb temperature coincident with a high outdoor air dry-bulb temperature. To help understand this issue, consider Bakersfield, CA when the outdoor dry- and wet-bulb temperatures respectively are $104^{\circ} \mathrm{F}$ and $70^{\circ} \mathrm{F}\left(40^{\circ} \mathrm{C}\right.$ and $\left.21^{\circ} \mathrm{C}\right)$. These temperatures correspond to the $0.4 \%$ design cooling condition (ASHRAE 2001). For these conditions, the lowest return-air wet-bulb temperature for which the superheat test can be used is $66^{\circ} \mathrm{F}\left(19^{\circ} \mathrm{C}\right)$. If the indoor air temperature is $75^{\circ} \mathrm{F}\left(24^{\circ} \mathrm{C}\right)$ and the humidity ratio is the same indoors and outdoors, then the corresponding indoor (return-air) wet-bulb temperature of $61^{\circ} \mathrm{F}\left(16^{\circ} \mathrm{C}\right)$ is too low to use the superheat test. Testing in the spring when outdoor air dry-bulb temperatures are not too hot can circumvent this problem. Alternatively, the indoor wet-bulb temperature can be elevated artificially by overheating and humidifying the house. This latter technique requires further development to determine appropriate strategies.

The superheat test also cannot be used in cool weather (outdoor air temperature less than $50^{\circ} \mathrm{F}$ or $10^{\circ} \mathrm{C}$ ). This limitation is primarily to protect the compressor from failure due to insufficient lubricant circulation under these conditions.

Equipment to carry out the superheat test includes a means of measuring various temperatures and a refrigerant pressure gauge. The cost of non-automated equipment to do this is about $\$ 400$ to $\$ 500$. 
Automated devices to carry out the superheat test, along with other fault detection diagnostics, are commercially available. However, they are expensive $(\$ 2,000$ to $\$ 20,000)$ and require further development. Wray et al. (2002) describe laboratory and field evaluations of two such devices.

\section{Procedural References}

At this time, there are no standards specifying temperature and pressure measurement accuracy or specific measurement locations for a superheat test. As a result, there can be significant variability in "measured" superheats. Example problems include measuring the indoor wet-bulb within the house rather than within the return plenum downstream of return duct leaks, measuring outdoor air temperature remotely from the condensing unit in direct sunlight with an unshielded sensor, and measuring refrigerant line temperature downstream of a line restriction or with an uninsulated sensor that has poor surface contact. Uncertainties in these measurements can easily lead to "measured" superheat errors of $10^{\circ} \mathrm{F}\left(6^{\circ} \mathrm{C}\right)$ or more. Laboratory test data from Farzad and O'Neal (1988) for capillary-tube-controlled equipment indicate a $10^{\circ} \mathrm{F}\left(6^{\circ} \mathrm{C}\right)$ superheat error can result in a charge assessment difference of about 5 to $9 \%$, depending on outdoor temperature. 


\section{Combustion Appliances}

\section{Backdrafting and Spillage Potential}

\section{Introduction}

While poor design or installation of either the combustion equipment or air-moving equipment can reduce efficiency, it can also lead to downdrafting, and possibly backdrafting with combustion gas spillage. Downdrafting is inward airflow from outdoors through a flue or chimney when no connected combustion appliance is operating. Appliance backdrafting is the failure of an operating combustion appliance to reverse a downdraft and to establish a proper flow of combustion gas products toward outdoors through the attached flue or chimney. Spillage is the entry of combustion gas products into the indoor air. Excessive depressurization of a house when exhaust devices are operating can cause some or all of these phenomena.

Substantial work has been carried out in Canada and the United States over the past 20 years in an attempt to understand backdrafting and spillage events related to combustion equipment venting and the operation of exhaust devices in houses, how long such events last, and how frequently they occur (Nagda et al. 1995). In spite of these efforts, we still lack the ability to reliably answer questions of whether backdrafting and spillage can occur, how long such events last and how frequently they occur (especially where stable backdrafting might occur), and whether the spillage gases are hazardous or toxic to occupants (Nagda 1995). However, although the frequency of spillage events might be very low, spillage directly affects the indoor environment and may pose significant health, comfort, or indoor air quality problems in some cases.

In particular, sustained backdrafting represents a catastrophic failure of the venting system and can cause serious health and safety problems. Very short periods of backdrafting are normally not hazardous because the small amount of combustion by-products released can be effectively diluted by whole-building ventilation. Unfortunately no standards or guidelines exist to determine how much backdrafting is acceptable, so it is generally best to avoid it completely.

\section{Applicable Metrics}

Familiar metrics for installation and operation of combustion appliances include safety issues such as clearance to combustibles, vent sizing, and outdoor airflow rates to support combustion. Performance metrics that describe the ability of an appliance to properly vent its combustion gases or conversely its potential for backdrafting and spillage of these gases into a house are less familiar. They include house depressurization or the draft (pressure differential) in the attached vent. These metrics can apply either to startup (cold flue) conditions or to steady-state operation. For the startup case, a particular metric is the cold-vent establishment pressure (CVEP), which represents the maximum indoor-outdoor pressure differential against which the hot combustion gases from the combustion appliance can establish a proper flow through the vent.

Two other important metrics involved with this issue are the concentrations of carbon monoxide $(\mathrm{CO})$ and nitrogen dioxide $\left(\mathrm{NO}_{2}\right)$ in the combustion gases. If the appliance backdrafts, exposure to elevated concentrations of $\mathrm{CO}$ indoors can be lethal to occupants, while exposure to $\mathrm{NO}_{2}$ can lead to chronic respiratory problems.

One other relevant metric is heat exchanger leakage, which involves the direct leakage of combustion gases into the space conditioning air flowing through the air-handling unit. This 
metric is more important for existing houses than for new houses. However, it may be desirable to check new equipment to detect manufacturing defects.

Apart from visually checking clearance to combustibles, vent sizing, and combustion air intake sizes, and measuring carbon monoxide concentrations at draft hoods of unsealed appliances, the potential for appliance backdrafting and spillage is the most critical one to assess in the course of residential commissioning. Heat exchanger leakage is also an important metric to evaluate, but there is no simple and reliable diagnostic at this time. Furthermore, the norm for heat exchanger leakage is poorly defined and may be unreliable for defining acceptable leakage levels of combustion gas products.

Although not directly related to appliance backdrafting and spillage, equipment control settings (e.g., a room thermostat setting, a water heater temperature setting, a high-limit or fan control setting) are simple metrics that are easy to evaluate. We recommend that these settings be checked during residential commissioning.

\section{Recommended Diagnostics}

Chapter 8 of the National Fuel Gas Code (NFPA 1999) describes procedures for use after appliance installation, but before placing the equipment in operation. These procedures include adjusting burner fuel input, adjusting the primary air, verifying ignition and safety control operation, and checking for "worst-case depressurization scenario" appliance backdrafting and spillage ("draft" test). Appendix H of the National Fuel Gas Code describes further procedures for the safety inspection of existing appliance installations, and includes detailed instructions for the "draft" test. ASTM Guide E1998-99 (1999b) describes similar procedures within its appliance downdrafting and backdrafting tests.

An advantage of all these tests is that they are simple, rapid, and require virtually no equipment, which makes them all useful as screening tools, especially when commissioning existing houses. However, except for the ASTM backdrafting test, they all pessimistically consider a weak downdraft that might be reversible as much a failure as a strong irreversible downdraft, and will fail some houses that do not have a problem. From a safety standpoint, this is preferable to the alternative of passing a house that does have a problem. Although the ASTM backdrafting test determines how long a combustion appliance takes to establish a draft after its burner fires, the prescribed time limits may not accurately represent the acceptability for backdrafting of the specific house/appliance combinations being tested. As a result, that test is a questionable improvement over the other tests.

The qualitative norms for the simple tests are not helpful in trying to resolve what changes should be made to the house and its equipment to meet a specified maximum depressurization that might cause appliance backdrafting and spillage. Two more detailed test protocols (CGSB 1995, ASTM 1999b) quantify the potential for depressurization-induced problems. Specifically, these tests are the house depressurization test, and the cold-vent establishment pressure (CVEP) test. However, even these more advanced tests are problematic: they can easily result in false positive or negative test results (Nagda and Koontz 2000). In spite of this, given that life safety and chronic health problems have been associated with combustion gas spillage, it is still better to use these imperfect methods than not.

We recommend that at least one of the two detailed tests be used during commissioning. The house depressurization test should be used as the primary diagnostic when testing time is a constraint, which is likely the usual case. The CVEP test is preferred if more time is available, 
because it might more accurately represent the potential for backdrafting and spillage for the specific house/appliance combinations being tested (Koontz and Nagda 1996). Because the house depressurization test does not involve operating the combustion appliances, we recommend that the National Fuel Gas Code test be used to supplement the house depressurization test when that latter test is used for commissioning. For the safety of the technician and house occupants, $\mathrm{CO}$ in the indoor air must be monitored during this test to assess whether hazardous conditions exist. The test must be terminated if such conditions occur.

- House Depressurization Test: The house depressurization test (CGSB 1995, ASTM 1999b) involves measuring the change in indoor-outdoor pressure differentials created by operating various combinations of installed air-moving equipment (space conditioning, ventilation, fireplace, and laundry) that can depressurize the house when combustion appliances are off. Measured changes in pressure differentials due to operating the air-moving equipment are compared with prescribed pressure limits to determine the potential for depressurizationinduced backdrafting and spillage. These limits are based on field tests in several Canadian houses.

The flue or chimney must be allowed to cool to within 5 to $10^{\circ} \mathrm{F}$ of the room air temperature after the combustion appliances are turned off and before the test begins. A blower door can be used to accelerate the cooling. Another prerequisite for the test is that the flue or chimney be checked for blockage. Qualitative diagnostics such as visual inspection (Oberholtzer 1993) and using a smoke tube, cigarette smoke, lit match, or lighter flame to identify the presence of a draft in the flue or chimney can be used. However, no diagnostics are currently used to quantify the restriction of a venting system. Duct leakage test diagnostics could be applied, but accessing the upper end of a flue or chimney to seal it temporarily can be difficult, potentially hazardous, and time consuming.

A disadvantage of this test is that the prescribed limits may not accurately represent the potential for backdrafting and spillage of the specific appliances in the house being tested.

Pressure gauges to carry out this test cost about $\$ 450$ to $\$ 750$.

- Cold Vent Establishment Pressure Test: The cold vent establishment pressure (CVEP) test (Koontz and Nagda 1996, Grimsrud et al. 1996, ASTM 1999b) involves similar conditions to the house depressurization test. This test also identifies the pressure limit at which each operating appliance establishes a draft in an initially cold flue or chimney, with the house airmoving systems and other combustion appliances off. A blower door is used to vary the depressurization of the house from $15 \mathrm{~Pa}$ or $25 \mathrm{~Pa}$ (whichever will induce actual backdrafting) toward zero until a draft is established. This element of the test is repeated for each combustion appliance, with the flue or chimney being allowed to cool to near the outdoor air temperature between each pressure limit subtest. A blower door can be used to accelerate the cooling. A smoke tube, cigarette smoke, lit match, or lighter flame is used to identify the stagnation or establishment of a draft in the flue or chimney and the cessation of any backdrafting.

Like the house depressurization test, this test measures the change in indoor-outdoor pressure differentials created by operating various combinations of installed air-moving equipment (space conditioning, ventilation, fireplace, and laundry) that can depressurize the house when combustion appliances are off. These measured changes in pressure differentials due to 
operating the air-moving equipment are compared with the measured CVEP limits to determine the potential for depressurization-induced backdrafting and spillage.

Grimsrud et al. 1996 indicate that two trained technicians can carry out this test and ancillary work (e.g., site characterization, blower door test, installation of long-term monitoring equipment) in one house over a period of 4 to 6 hours. A computerized data acquisition system that monitors the necessary pressure difference and that adjusts the blower door fan speed to maintain each desired envelope pressure difference can be used to facilitate the CVEP test, especially when determining the CVEP limits. A temperature sensor appropriately located could be used as a backdrafting indicator when a data acquisition system is used. With this automation, the CVEP test itself should take about 3 hours. As with the DeltaQ duct leakage test, there can be a tradeoff between increased capital costs for automating the test and the time savings resulting from the automation. Together, data acquisition systems and computers to record the data cost about $\$ 3,200$ to $\$ 4,500$. However, the time saving, record keeping ability, and operator error reductions resulting from automating the test outweigh the higher capital cost of this option. Without automation, pressure gauges to carry out this test cost about $\$ 450$ to $\$ 750$.

This test has the advantage that it does not use prescribed limits like the house depressurization test or appliance backdrafting test. Consequently, its results might more accurately represent the potential for depressurization-induced backdrafting and spillage of the specific appliances in the house being tested (Koontz and Nagda 1996).

For the safety of the technician and house occupants, $\mathrm{CO}$ in the indoor air must be monitored during this test to assess whether hazardous conditions exist. The test must be terminated if such conditions occur.

\section{Procedural References}

1. NFPA. 1999. "NFPA 54-1999: National Fuel Gas Code”. Quincy, MA: National Fire Protection Association. http://www.nfpa.org/catalog/.

2. ASTM. 1999b. “ASTM Guide E1998-99, Standard Guide For Assessing DepressurizationInduced Backdrafting and Spillage From Vented Combustion Appliances”. Philadelphia, PA; American Society for Testing and Materials. http://www.astm.org.

3. CGSB. 1995. "CAN/CGSB-51.71-95, Method To Determine The Potential For PressureInduced Spillage From Vented, Fuel-Fired, Space Heating Appliances, Water Heaters and Fireplaces". Ottawa, CA; Canadian General Standards Board. http://www.pwgsc.gc.ca/cgsb/catalogue/specs/051/051_071-e.html. 


\section{ACKNOWLEDGEMENTS}

This report describes work supported by the California Energy Commission through the Public Interest Energy Research program under contract no. 500-98-033, and by the Assistant Secretary for Energy Efficiency and Renewable Energy, Building Technologies Program, of the U.S. Department of Energy under contract no. DE-AC03-76SF00098.

The authors wish to acknowledge the significant contributions of LBNL staff who were involved in analytical and experimental work that directly supported our preparation of this guide: Bass Abushakra, Darryl Dickerhoff, Nance Matson, Jennifer McWilliams, Jeff Siegel, Duo Wang, and Jacob Wempen. The authors also thank the CEC staff and members of the Project Advisory Committee for their oversight of the work leading up to and including this guide.

\section{REFERENCES}

ACCA. 1995. “ACCA Manual S - Residential Equipment Selection”. Washington, DC: Air Conditioning Contractors of America.

American Lung Association. 1999. "Asthma in Children Fact Sheet". Asthma Web Site. http://www.lungusa.org/asthma/ascpedfac99.html. September 1999.

ASHRAE. 1994. "ANSI/ASHRAE Standard 119-1988 (RA 94), Air Leakage Performance for Detached Single-Family Residential Buildings". Atlanta, GA; American Society of Heating, Refrigerating, and Air-Conditioning Engineers, Inc.

ASHRAE. 1999. "BSR/ASHRAE Draft Standard 152P-1999, Method of Test for Determining the Design and Seasonal Efficiencies of Residential Thermal Distribution Systems". Atlanta, GA; American Society of Heating, Refrigerating, and Air-Conditioning Engineers, Inc.

ASHRAE. 2001a. "ASHRAE Handbook - Fundamentals". Atlanta, GA: American Society of Heating, Refrigerating, and Air-Conditioning Engineers, Inc.

ASHRAE. 2001b. “ANSI/ASHRAE Standard 136-1993 (RA 2001), A Method of Determining Air Change Rates in Detached Dwellings". Atlanta, GA; American Society of Heating, Refrigerating, and Air-Conditioning Engineers, Inc.

ASHRAE. 2002. "ASHRAE Standard 62.2P - Ventilation and Acceptable Indoor Air Quality in Low-Rise Residential Buildings". Atlanta, GA: American Society of Heating, Refrigerating and Air-Conditioning Engineers, Inc. April.

ASTM. 1990. “ASTM Standard C1060-90, Standard Practice for Thermographic Inspection of Insulation Installations in Envelope Cavities of Frame Buildings". Philadelphia, PA; American Society for Testing and Materials.

ASTM. 1994. “ASTM Standard E1554-94, Standard Test Methods for Determining External Air Leakage of Air Distribution Systems by Fan Pressurization”. Philadelphia, PA; American Society for Testing and Materials.

ASTM. 1995. "ASTM Standard E1186-87, Standard Practices for Air Leakage Site Detection in Building Envelopes”. Philadelphia, PA; American Society for Testing and Materials.

ASTM. 1997. “ASTM Standard E1827-96, Standard Test Methods for Determining Airtightness of Buildings Using an Orifice Blower Door". Philadelphia, PA; American Society for Testing and Materials. 
ASTM. 1998. "ASTM Standard D4444-92, Standard Test Methods for Use and Calibration of Handheld Moisture Meters". West Conshohocken, PA: American Society for Testing and Materials.

ASTM. 1999a. "ASTM Standard E779-99, Standard Test Method for Determining Air Leakage Rate by Fan Pressurization”. Philadelphia, PA; American Society for Testing and Materials.

ASTM. 1999b. "ASTM Guide E1998-99, Standard Guide For Assessing DepressurizationInduced Backdrafting and Spillage From Vented Combustion Appliances". Philadelphia, PA; American Society for Testing and Materials.

Blasnik, M., T. Downey, J. Proctor, and G. Peterson.1996. "Assessment of HVAC Installations in New Homes in APS Service Territory". Proctor Engineering Group Report for Arizona Public Service Company.

Building America. 2000. "Building America: Systems Engineering". August 29. http://www.eren.doe.gov/buildings/building_america/system.shtml.

Carmody, J., S. Selkowitz, D. Arasteh, and L. Heschong. 2000. "Residential Windows: A Guide to New Technologies and Energy Performance". 2nd Edition. New York: W.W. Norton \& Company.

CEC. 1998. "Low-Rise Residential Alternative Calculation Method Approval Manual for 1998 Energy Efficiency Standards for Low-Rise Residential Buildings". Sacramento, CA: California Energy Commission. http://www.energy.ca.gov/title24/residential_acm/index.html.

CGSB. 1986. "CAN/CGSB-149.10-M86, Determination of the Airtightness of Building Envelopes by the Fan Depressurization Method". Ottawa, CA; Canadian General Standards Board.

CGSB. 1995. "CAN/CGSB-51.71-95, Method To Determine The Potential For Pressure-Induced Spillage From Vented, Fuel-Fired, Space Heating Appliances, Water Heaters and Fireplaces". Ottawa, CA; Canadian General Standards Board.

Christian, J.E. and J. Kosny. 1995. "Toward a National Opaque Wall Rating Label". Proceedings of Thermal Performance of the Exterior Envelopes of Buildings VI, Clearwater Beach, FL, pp. 221-239. Atlanta, GA; American Society of Heating, Refrigerating, and Air-Conditioning Engineers, Inc.

Christian, J.E., J. Kosny, A.O. Desjarlais, and P.W. Childs. 1998. "The Whole Wall Thermal Performance Calculator - On the Net". Proceedings of the ASHRAE Thermal Performance of the Exterior Envelopes of Buildings VII Conference. Clearwater Beach, FL. Dec 6-10. pp.287-299.

ConSol. 1999. "CIEE Final Project Report: Protocols for Energy Efficient Residential Building Envelopes". Stockton, CA; ConSol.

ConSol. 2000. "Energy Efficient Residential New Construction: Market Transformation, Spectrally Selective Glass". Report to the US Department of Energy, Contract No. DE-FG0199EE27585. Stockton, CA; ConSol. December.

Davis Energy Group. 2000. "Residential Construction Quality Assessment Project: Phase I Final Report". Report to the California Energy Commission, CEC Report P400-00-022. November.

Downey, T. and J. Proctor. 1999. "Residential Central Air Conditioner Service Program Final Report". Report of Proctor Engineering Group to Pacific Gas \& Electric Company. January 29. 
EDTM. 2000. "Manual IG Thickness Gauge, \#MG1500”. Electronic Design To Market, Inc. website http://www.edtm.com/mg1500.htm.

EIMA. 2000. "EIMA Guidelines \& Checklist". Morrow, GA: EIFS Industry Members Association. http://www.dryvit.com/eima.htm. November 1.

ESB. 1997. "Finding the No Extra Cost Energy-Efficient Home". Energy Source Builder \#51. June.

Farzad, M. and D.L. O'Neal. 1988. “An Evaluation of Improper Refrigerant Charge on the Performance of a Split System Air Conditioner with Capillary Tube Expansion". Texas A\&M University, Energy Systems Laboratory Report ESL-TR-88/07-01. July.

Francisco, P.W. 2000. Personal Communication with Craig Wray. August.

Francisco, P. and L. Palmiter. 1999. "Field Validation of ASHRAE Standard 152”. Final Report, ASHRAE Project 1056-RP.

Francisco, P.W. and L. Palmiter. 2000. "Performance of Duct Leakage Measurement Techniques in Estimating Duct Efficiency: Comparison to Measured Results". Proceedings, 2000 ACEEE Summer Study on Energy Efficient Buildings. Washington, D.C.; American Council for an Energy-Efficient Economy.

GAHI. 2000. "The GAHI Protocol for Exterior Insulation Finishing Systems (EIFS) Moisture Intrusion Inspections - One \& Two Family Homes". Atlanta, GA: Georgia Association of Home Inspectors. http://www.gahi.com/eifsprot.html. November 1.

Griffith, B.T. 1999. "Field Verification of Components: Hand-Held Detector for Low-E and Spectrally Selective Coatings". Berkeley, CA; Lawrence Berkeley National Laboratory, Environmental Energy Technologies Division, Building Technologies Department, Windows \& Daylighting Group website http://windows.lbl.gov/win prop/component_verification.

Grimsrud, D.T., M.D. Koontz, and N.L. Nagda. 1996. "Field Protocol for Determining Depressurization-Induced Backdrafting and Spillage from Vented Residential Gas Appliances (Version 2.0)". Report of Minnesota Building Research Center, University of Minnesota; ENERGEN Consulting, Inc.; and GEOMET Technologies, Inc. to Gas Research Institute and Pacific Gas and Electric Company, GRI-95/0303. August.

Halvorsen, D., K. Youso, and J. Buchta. 2000. "Special Report: Water Intrusion Can Silently Destroy Houses". Minneapolis Star Tribune, June 18.

HEM. 1998. "5 Steps to Tract Home Success". Home Energy Magazine. March/April.

James, W.L. 1988. "Electric Moisture Meters for Wood." General Technical Report FPL-GTR-6. Madison, WI: U.S. Department of Agriculture, Forest Service, Forest Products Laboratory.

Jump, D.A., I.S. Walker, and M.P. Modera. 1996. "Field Measurements of Energy Efficiency and Duct Retrofit Effectiveness in Residential Forced Air Distribution Systems". Proceedings, 1996 ACEEE Summer Study on Energy Efficient Buildings. Washington, D.C.: American Council for an Energy-Efficient Economy.

Knight, R.L. and G. Thomas. 2000. "Whole-House Contractor Team Accreditation:

Development of a Feasible Model and Implementation Process". Report of Performance Systems Development, Inc. and Bevilacqua-Knight, Inc. to California Energy Commission. CEC P40000-013CR. July. 
Koles, G., R. Hitchcock, and M.H. Sherman. 1996. "Metrics for Building Performance Assurance". Lawrence Berkeley Laboratory report LBL-39203.

Koontz, M.D. and N.L. Nagda. 1996. "Protocols for Assessing Pressure-Induced Spillage from Gas-Fired Furnaces and Water Heaters (Version 1.1)". Report of ENERGEN Consulting, Inc. and GEOMET Technologies, Inc. to Gas Research Institute and Pacific Gas and Electric Company, GRI-95/0266. July.

Lstiburek, J. and J. Carmody. 1994. "Moisture Control Handbook: Principles and Practices for Residential and Small Commercial Buildings". New York: Van Nostrand Reinhold.

Lovins, A. 1999. "Greening the Building and the Bottom Line". RSA Environment Lectures 1999/2000, Sustainability Action Group exchange, RSA at Bristol. http://www.sagersa.org.uk/lectures/texts/amorybuilding.html.

LVR-J. 2000. "Pulte Homes Commits to Energy Efficiency". Las Vegas Review-Journal and Las Vegas SUN. April 30. http://www.lvrjsun.com/realestate/REApr-30-Sun2000/All/13450419.html.

Margotta, P., J. Corrasi, and J. Craig. 1984. "Wagner Model L-600 Moisture Meter Comparison". Wood Technology Program, University of Massachusetts at Amherst.

Matson, N.E., C.P. Wray, I.S. Walker, and M.H. Sherman. 2002. "Potential Benefits of Commissioning California Homes". Lawrence Berkeley National Laboratory report LBNL48258. http://epb1.lbl.gov/EPB/Publications/lbnl-48258.pdf.

Modera, M.P. and R. Jansky. 1992. "Residential Air-Distribution Systems: Interactions with the Building Envelope". ASHRAE Thermal Performance of the Exterior Envelopes of Buildings V. Conference Proceedings, Florida, December, 623-631.

Modera, M.P. and D.J. Wilson. 1990. "The Effects of Wind on Residential Building Leakage Measurements". ASTM STP 1067, Air Change Rate and Air Tightness in Buildings, Philadelphia, PA: American Society for Testing and Materials, pp. 132-145. LBL-24195.

Nagda, N.L. 1995. "Workshop on Depressurization, Backdrafting and Indoor Air Quality: Issues and Research Needs". Report of ENERGEN Consulting, Inc. to Gas Research Institute and Pacific Gas and Electric Company, GRI-95/0403. October.

Nagda, N.L., R.W. Krug, and M.D. Koontz. 1995. "Depressurization, Backdrafting, and Spillage from Vented Gas Appliances - A Literature Review”. Report of ENERGEN Consulting, Inc. and GEOMET Technologies, Inc. to Gas Research Institute and Pacific Gas and Electric Company, GRI-95/0426. November.

Nagda, N.L. and M. Koontz. 2000. "Rationale for Proposed Method for Assessing Backdrafting and Spillage". Presentation at ASTM E6.41 - Mechanical Systems Meeting, Toronto, ON. April 11.

Neal, C.L. 1998. "Field Adjusted SEER [SEERFA] Residential Buildings: Technologies, Design, and Performance Analysis". Proceedings of the 1998 ACEEE Summer Study on Energy Efficient Buildings, 1.197. Washington, D.C.; American Council for an Energy-Efficient Economy.

Nelson, G. 2001. Personal Communication with Craig Wray. March. 
NFPA. 1999. “NFPA 54-1999: National Fuel Gas Code”. Quincy, MA: National Fire Protection Association.

NFRC. 1993. "NFRC 301-93: Standard Test Method for Emittance of Specular Surfaces Using Spectrometric Measurements”. Silver Spring, MD: National Fenestration Rating Council.

NHCID. 1998. "Moisture Testing Guide for Wood Frame Construction Clad with Exterior Insulation and Finish Systems (EIFS), Version 3.01". Prepared for EIFS Review Committee. Wilmington, NC: New Hanover County Inspection Department. August 4.

Oberholtzer, D.L. 1993. "Chimney Diagnostics for Safer and Healthy Appliance Venting”. Proceedings of the ASHRAE Indoor Air Quality Conference '93, Philadelphia, PA, pp. 249-253. Atlanta, GA; American Society of Heating, Refrigerating, and Air-Conditioning Engineers, Inc.

Pacific Consulting Services. 2000. "Market Assessment \& Evaluation Study in Support of Codes and Standards". Final Report of Pacific Consulting Services, Davis Energy Group, and Eley Associates to Pacific Gas \& Electric. PG\&E Study ID number 411. August 31.

Palmiter, L. and P.W. Francisco. 2000. "Development of a Simple Device for Field Air Flow Measurement of Residential Air Handling Equipment: Phase II”. Final Report of Ecotope, Inc. to the U.S. Department of Energy, STTR Grant \#DE-FG03-97ER86060. June.

RDH. 2000. "Building Envelope Rehabilitation: Consultant Guide”. Report of RDH Building Engineering Limited to Canada Mortgage and Housing Corporation. Ottawa, ON: Canadian Housing Information Centre. April.

Rocky Mountain Institute. 1997. "Tunneling Through the Cost Barrier." In Rocky Mountain Institute Newsletter. Summer. pp. 1-4.

Rodriguez, A.G. 1995. "Effect of Refrigerant Charge, Duct Leakage, and Evaporator Air Flow on the High Temperature Performance of Air Conditioners and Heat Pumps". Texas A\&M Energy Systems Laboratory, ESL-TH-95/08-01.

Sherman, M.H., R.F. Szydlowski, P.G. Cleary, M.P. Modera, and M.D. Levine. 1987. "Development and Implementation of Survey Techniques for Assessing In-Situ Appliance Efficiencies”. Lawrence Berkeley Laboratory report LBL-23455.

Sherman, M.H. and L. Palmiter. 1994. "Uncertainties in Fan Pressurization Measurements". In Airflow Performance of Envelopes, Components and Systems, Philadelphia, American Society for Testing and Materials (STP 1255), Modera \& Persily Eds., pp. 262-283, 1994. LBL-32115.

Sherman, M.H. and D.J. Wilson. 1986. "Relating Actual and Effective Ventilation in Determining Indoor Air Quality". Lawrence Berkeley National Laboratory report LBL-20424.

Siegel, J. and B. Manclark. 1998. "Pressure Pans: New Uses and Old Fundamentals". Home Energy Magazine, Vol. 15, No. 1, January/February 1998 pp. 37-42. Berkeley, CA: Energy Auditor \& Retrofitter, Inc.

Simpson, W.T. 1999. "Chapter 12: Drying and Control of Moisture Content and Dimensional Changes, Wood Handbook - Wood as an Engineering Material." General Technical Report FPLGTR-113. Madison, WI: U.S. Department of Agriculture, Forest Service, Forest Products Laboratory.

Uniacke, M. 2000. “Cheating - The Insulation Industry's Dirty Secret”. Berkeley, CA: Home Energy Magazine, November / December 2000. pp. 24-30. 
Walker, I., M. Modera, A. Tuluca, and I. Graham. 1996. "Energy Effectiveness of Duct Sealing and Insulation in Two Multifamily Buildings". Proceedings of 1996 ACEEE Summer Study, Asilomar, CA. Washington, D.C.; American Council for an Energy-Efficient Economy.

Walker, I., M. Sherman, M. Modera, and J. Siegel. 1998a. "Leakage Diagnostics, Sealant Longevity, Sizing and Technology Transfer in Residential Thermal Distribution Systems". Lawrence Berkeley National Laboratory report LBNL-41118. http://epb1.lbl.gov/EPB/Publications/lbl-41118.pdf.

Walker, I.S., J. Siegel, K. Brown, and M.H. Sherman. 1998b. "Saving Tons at the Register". Proceedings of 1998 ACEEE Summer Study, Pacific Grove, CA. Washington, D.C.; American Council for an Energy-Efficient Economy. Also Lawrence Berkeley National Laboratory report LBNL-41957. http://epb1.lbl.gov/EPB/Publications/lbnl-41957.pdf.

Walker, I., M. Sherman, M. Modera, and J. Siegel. 1998c. "Leakage Diagnostics, Sealant Longevity, Sizing and Technology Transfer in Residential Thermal Distribution Systems: Part II’. Lawrence Berkeley National Laboratory report LBNL-42691.

Walker, I., M. Sherman, and J. Siegel. 1999. "Distribution Effectiveness and Impacts on Equipment Sizing". Lawrence Berkeley National Laboratory report LBNL 43724. http://epb1.lbl.gov/EPB/Publications/lbnl-43724.pdf.

Walker, I.S., Sherman, M.H., Wempen, J., Wang, D., McWilliams, J.A., and Dickerhoff, D.J. 2001. “Development of a New Duct Leakage Test: Delta Q". Lawrence Berkeley National Laboratory report LBNL- 47308. http://epb1.lbl.gov/EPB/Publications/lbnl-47308.pdf.

Walker, I.S. 2001. “California's Duct Opportunity”. Lawrence Berkeley National Laboratory website. http://ducts.lbl.gov/calducts.htm\#peak.

Warren, S.R. (editor). 1994. “ASTM Hand-Held Moisture Meter Workshop”. Madison, WI: Forest Products Society.

Wilcox, B., K. Nittler, D.A. Ross, and J. Proctor. 2001. "Split System Space Cooling Refrigerant Charge and Airflow Measurement". Contractors Report, California energy Commission Publication No. P 400-01-014. March 20.

http://www.energy.ca.gov/reports/2001-03-20 400-01-014.PDF.

Wirtshafter Associates. 2000. "Residential Contractor Program Evaluation, Phase II Final Report”. Quakertown, PA: Volume 1, Summary Report. July 10.

Wray, C.P., M.A. Piette, M.H. Sherman, R.M. Levinson, N.E. Matson, D.A. Driscoll, J.A. McWilliams, T.T. Xu, and W.W. Delp. 2000. "Residential Commissioning: A Review of Related Literature". Lawrence Berkeley National Laboratory report LBNL-44535. http://epb1.lbl.gov/EPB/Publications/lbnl-44535.pdf.

Wray, C.P., I.S. Walker, J.A. Siegel, and M.H. Sherman. 2002. "Practical Diagnostics for Evaluating Residential Commissioning Metrics" Lawrence Berkeley National Laboratory report LBNL-45959. http://epb1.lbl.gov/EPB/Publications/lbnl-45959.pdf.

Yuill, G.K. 1986. "The Variation of the Effective Natural Ventilation Rate with Weather Conditions". Renewable Energy Conference '86, Solar Energy Society of Canada Inc., pp.70-75.

Yuill, G.K. 1991. "The Development of a Method of Determining Air Change Rates in Detached Dwellings for Assessing Indoor Air Quality”. ASHRAE Transactions, Vol. 97, Part 2. 


\section{APPENDIX II. EXAMPLE AUDIT AND DIAGNOSTIC PROCESS}

Jennifer McWilliams, Craig Wray, Max Sherman, and Iain Walker

Lawrence Berkeley National Laboratory

Berkeley, California 


\section{OVERVIEW}

This appendix provides a flow chart to illustrate a possible sequence for carrying out tasks in the initial audit and diagnostic phase of a whole-house residential commissioning process.

Supporting text in Table II-1 summarizes the commissioning tasks by describing what each task does, what test protocols could be used, what equipment is needed, how long each task takes, and the potential energy savings associated with carrying out each task. The task numbers in Table II-1 correspond to the numbering used in the flow chart. More details about the commissioning process and these tasks are described in Appendix I "Guidelines for Residential Commissioning". Appendix III provides a sample commissioning report that could be used to document the audit and diagnostic findings from commissioning a house using the sequence of tasks (and numbering) described here. The data included in the report are for an actual house in Concord, California, which has been retrofitted recently as part of a U.S. Department of Energy (DOE) demonstration of residential commissioning.

In general, we do not recommend that potential energy savings from commissioning be assessed on a component-by-component or task basis. Instead, the package of proposed retrofits should be addressed as a system because of the significant interactions between components. Component estimates are difficult to estimate individually, because they are highly dependent on the type of house and equipment that is being commissioned, on the quality of the construction and installations, and on the order in which retrofits are applied (returns from improvements diminish as retrofits are incrementally applied). Not withstanding these caveats and difficulties, it is still somewhat useful to roughly disaggregate the categorical energy savings for the various commissioning tasks to understand their relative generic importance. Consequently, we have listed potential savings as high (e.g., for duct sealing) when we believe that there is a potential for more than $10 \%$ savings through component improvements; medium corresponds to potential savings less than this. A low category is not used (which could be taken to mean little or no potential energy savings). For cases where such a category could be used (e.g., combustion spillage tests), there are overriding health and safety issues that take precedence, and we have noted them as such.

It is important to recognize that diagnostics involving airflow and pressure measurements must be carried out one at a time. To estimate the time required to carry out multiple tests of this type, one needs to add the individual times listed. In some cases, there may be some minor time reductions associated with having equipment already setup (e.g., 5 to 10 minutes). An example is measuring envelope airtightness with a blower door and then duct leakage only to outside using the duct pressurization test, which requires the use of the blower door as well. One exception is the DeltaQ test, which simultaneously measures envelope airtightness and duct leakage. Other diagnostics such as insulation inspection or window characterization can be carried out while these types of tests are underway, as long as envelope airtightness remains undisturbed during the test.

Also, in many cases, the equipment from one diagnostic can be used for other diagnostics, often with only slight modifications. An example is the envelope airtightness test and DeltaQ duct leakage test, where both tests use the same equipment (the DeltaQ test uses a blower door and a pressure measurement device to simultaneously determine envelope airtightness and duct leakage). Another example is using the fan-assisted flow meter to determine air-handler airflow and the fan-assisted flow hood to determine duct airflows. Both devices use a fan-assisted flow meter; the latter also has a flow capture hood. 
We have included the temperature split method of assessing air-handler airflow in the flow chart for completeness because it is currently described in California Title 24. However, we do not recommend its use, because it is prone to errors of more than $20 \%$ in many cases and nearly a factor of two in worst cases, due to flow nonuniformities and radiant effects (Palmiter and Francisco 2000). It is particularly problematic when used to assess airflows in systems that have inadequate refrigerant charge, because of the wide variation in air temperatures downstream of the evaporator coil (Wray et al. 2002). 
EXAMPLE AUDIT AND DIAGNOSTIC TASK SEQUENCE

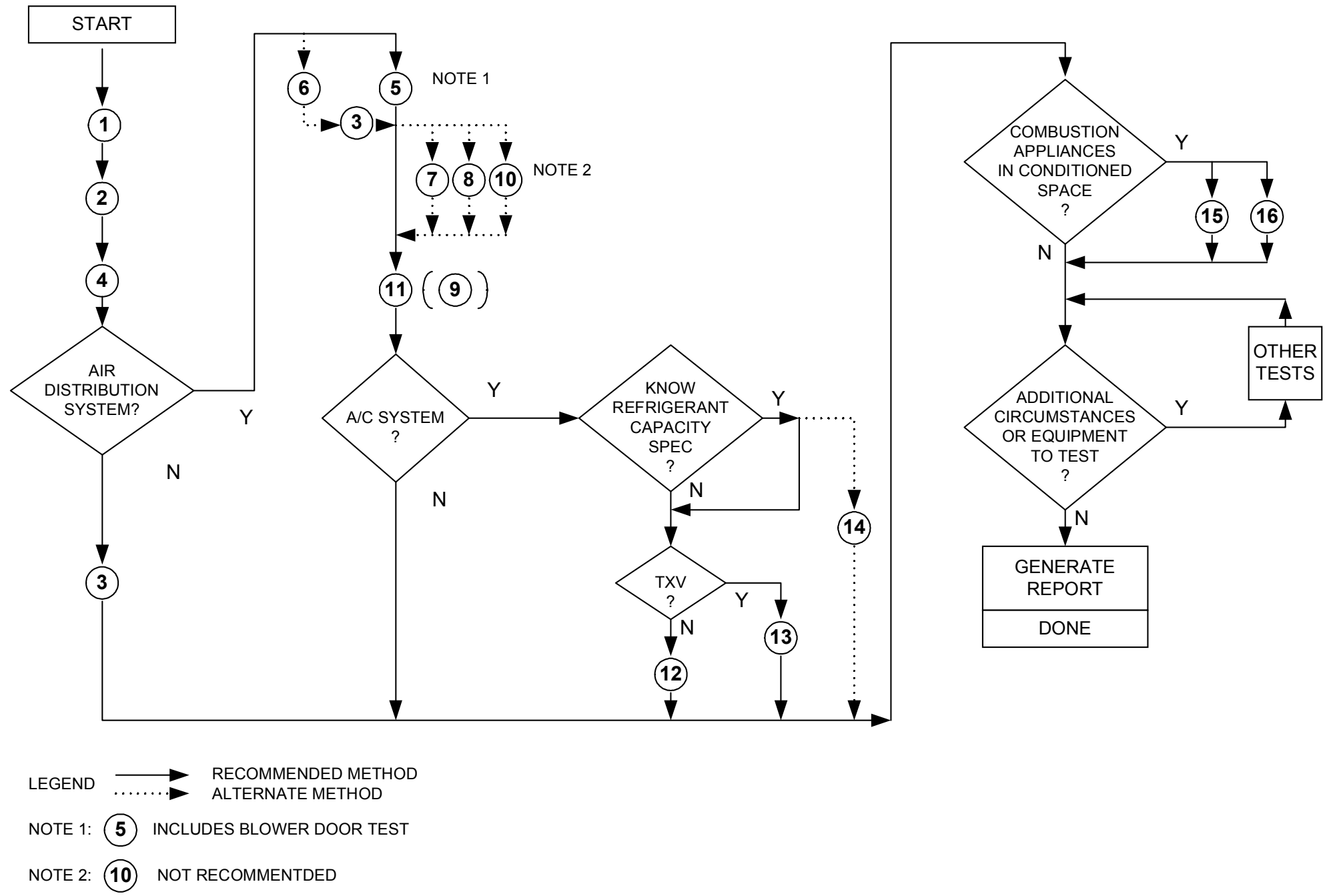


Table II-1. Recommended Audit and Diagnostic Procedures

\begin{tabular}{|c|c|c|c|c|c|c|}
\hline Task & Name & Description & Protocol & Equipment & $\begin{array}{l}\text { Time } \\
\text { (min.) }\end{array}$ & $\begin{array}{l}\text { Energy } \\
\text { Savings } \\
\text { Potential }\end{array}$ \\
\hline 1 & $\begin{array}{l}\text { Insulation } \\
\text { Inspection }\end{array}$ & $\begin{array}{l}\text { Check that the wall, attic, and floor } \\
\text { insulation installation is in } \\
\text { accordance with specifications. }\end{array}$ & $\begin{array}{l}\text { ConSol 1999, "CIEE Final Project Report: } \\
\text { Protocols for Energy Efficient Residential Building } \\
\text { Envelopes" }\end{array}$ & $\begin{array}{l}\text { Toolbox, infrared } \\
\text { camera. }\end{array}$ & $30-60$ & Medium \\
\hline 2 & $\begin{array}{l}\text { Window } \\
\text { Inspection }\end{array}$ & $\begin{array}{l}\text { Check that the window type } \\
\text { (emittance and glazing gap) is in } \\
\text { accordance with specifications. }\end{array}$ & $\begin{array}{l}\text { No standards. Use equipment manufacturer's } \\
\text { instructions. }\end{array}$ & $\begin{array}{l}\text { Handheld window } \\
\text { inspection tools. }\end{array}$ & 30 & Medium \\
\hline 3 & $\begin{array}{l}\text { Envelope } \\
\text { Airtightness Test } \\
\text { and Leak } \\
\text { Detection }\end{array}$ & $\begin{array}{l}\text { Determine the air tightness of the } \\
\text { building envelope and determine the } \\
\text { location of leaks. }\end{array}$ & ASTM E779-99, E1827-96, and E1186-87. & $\begin{array}{l}\text { Blower door or } \\
\text { equivalent and } \\
\text { smoke stick. }\end{array}$ & $30-60$ & Medium \\
\hline 4 & $\begin{array}{l}\text { Envelope Moisture } \\
\text { Test }\end{array}$ & $\begin{array}{l}\text { Check that construction details will } \\
\text { not lead to moisture problems later } \\
\text { on. The inspector must be } \\
\text { knowledgeable about common } \\
\text { moisture problems in the region. }\end{array}$ & $\begin{array}{l}\text { No general standards exist. Available resources } \\
\text { include EIFS inspection protocols from North } \\
\text { Carolina and Georgia (NHCID } 1998 \text { and GAHI } \\
\text { 2000), plus construction guidelines by Lstiburek } \\
\text { and Carmody (1994) and RDH Building } \\
\text { Engineering (2000). }\end{array}$ & $\begin{array}{l}\text { Surface scanning } \\
\text { dielectric meter } \\
\text { and conductance } \\
\text { probe. }\end{array}$ & $30-60$ & $\begin{array}{l}\text { Health and } \\
\text { Safety }\end{array}$ \\
\hline 5 & $\begin{array}{l}\text { DeltaQ Duct } \\
\text { Leakage Test }\end{array}$ & $\begin{array}{l}\text { Determine the building envelope } \\
\text { leakage and duct leakage (at } \\
\text { operating conditions) using a } \\
\text { combined test. }\end{array}$ & Walker et al. 2001. & $\begin{array}{l}\text { Blower door or } \\
\text { equivalent. }\end{array}$ & 30 & High \\
\hline 6 & $\begin{array}{c}\text { Duct } \\
\text { Pressurization }\end{array}$ & $\begin{array}{l}\text { Determine the duct leakage at a given } \\
\text { pressure across the ducts. }\end{array}$ & ASTM E1554-94 & $\begin{array}{l}\text { Fan-assisted flow } \\
\text { meter. }\end{array}$ & 30 & High \\
\hline 7 & $\begin{array}{l}\text { Air-Handler } \\
\text { Airflow: Fan- } \\
\text { Assisted Flow } \\
\quad \text { Meter }\end{array}$ & $\begin{array}{l}\text { Determine the airflow across the air } \\
\text { handler by redirecting all of the flow } \\
\text { through a calibrated flow meter. }\end{array}$ & No standards. See CEE (2000) Section 3.13.1. & $\begin{array}{l}\text { Fan-assisted flow } \\
\text { meter. }\end{array}$ & 30 & Medium \\
\hline 8 & $\begin{array}{c}\text { Air-Handler } \\
\text { Airflow: Plate and } \\
\text { Grid }\end{array}$ & $\begin{array}{l}\text { Determine the airflow across the air } \\
\text { handler by inserting a calibrated flow } \\
\text { plate into the filter slot. }\end{array}$ & Manufacturer's instructions. & $\begin{array}{l}\text { Calibrated flow } \\
\text { plate. }\end{array}$ & 30 & Medium \\
\hline
\end{tabular}




\section{Table II-1 (continued). Recommended Audit and Diagnostic Procedures}

\begin{tabular}{|c|c|c|c|c|c|c|}
\hline Task & Name & Description & Protocol & Equipment & $\begin{array}{l}\text { Time } \\
\text { (min.) }\end{array}$ & $\begin{array}{l}\text { Energy } \\
\text { Savings } \\
\text { Potential }\end{array}$ \\
\hline 9 & $\begin{array}{l}\text { Air-Handler } \\
\text { Airflow: Sum-of- } \\
\text { Registers }\end{array}$ & $\begin{array}{l}\text { Determine the airflow across the air-handler } \\
\text { by adding up the duct leakage plus the flow } \\
\text { through the grilles. This can be done on } \\
\text { either the supply or return side of the system. }\end{array}$ & $\begin{array}{l}\text { No standards. See CEE (2000) Section } \\
\text { 3.13.3. }\end{array}$ & $\begin{array}{l}\text { Fan-assisted flow } \\
\text { hood (needs a duct } \\
\text { leakage test also). }\end{array}$ & 60 & Medium \\
\hline $10 *$ & $\begin{array}{l}\text { Air-Handler } \\
\text { Airflow: } \\
\text { Temperature Split }\end{array}$ & $\begin{array}{l}\text { Assess the airflow across the air-handler by } \\
\text { measuring upstream and downstream air } \\
\text { temperatures. Does not measure airflow. }\end{array}$ & $\begin{array}{l}\text { No standards. See CEE (2000) Section } \\
\text { 3.13.2. }\end{array}$ & Temperature sensors. & 30 & Medium \\
\hline 11 & $\begin{array}{l}\text { Supply Register } \\
\text { Airflows }\end{array}$ & $\begin{array}{l}\text { Determine whether the airflow into each } \\
\text { room is in accordance with specifications. }\end{array}$ & $\begin{array}{l}\text { No standards. Use equipment } \\
\text { manufacturer's instructions. }\end{array}$ & $\begin{array}{l}\text { Fan assisted flow } \\
\text { hood. }\end{array}$ & 60 & Medium \\
\hline 12 & $\begin{array}{c}\text { Superheat } \\
\text { Refrigerant } \\
\text { Charge Test }\end{array}$ & $\begin{array}{l}\text { Assess the refrigerant charge level for fixed- } \\
\text { orifice-controlled cooling equipment. }\end{array}$ & $\begin{array}{l}\text { No standards. See CEE (2000) Section } \\
\text { 3.14.1. }\end{array}$ & $\begin{array}{l}\text { Refrigerant gauge } \\
\text { set, temperature } \\
\text { sensors. }\end{array}$ & 60 & High \\
\hline 13 & $\begin{array}{l}\text { Subcooling } \\
\text { Refrigerant } \\
\text { Charge Test }\end{array}$ & $\begin{array}{l}\text { Assess the refrigerant charge level for TXV- } \\
\text { controlled cooling equipment. }\end{array}$ & $\begin{array}{l}\text { No standards. See CEE (2000) Section } \\
\text { 3.14.2. }\end{array}$ & $\begin{array}{l}\text { Refrigerant gauge } \\
\text { set, temperature } \\
\text { sensors. }\end{array}$ & 60 & High \\
\hline 14 & Gravimetric Test & $\begin{array}{l}\text { Determine the amount of refrigerant charge } \\
\text { present in the cooling equipment. }\end{array}$ & $\begin{array}{l}\text { No standards. See CEE (2000) Section } \\
\text { 3.14.4. }\end{array}$ & $\begin{array}{l}\text { Refrigerant recovery } \\
\text { equipment, vacuum } \\
\text { gauge, vacuum } \\
\text { pump, charging } \\
\text { scale. }\end{array}$ & 120 & High \\
\hline 15 & $\begin{array}{c}\text { House } \\
\text { Pressurization } \\
\text { Spillage Test }\end{array}$ & $\begin{array}{l}\text { Assess whether house depressurization } \\
\text { caused by air moving appliances in a house } \\
\text { could cause combustion appliance spillage, } \\
\text { compared to generic norms. }\end{array}$ & $\begin{array}{l}\text { NFPA 54-1999, ASTM Guide E1998-99, } \\
\text { CAN/CGSB-51.71-95. }\end{array}$ & $\begin{array}{l}\text { Digital pressure } \\
\text { sensor. }\end{array}$ & 30 & $\begin{array}{l}\text { Health and } \\
\text { Safety }\end{array}$ \\
\hline 16 & $\begin{array}{l}\text { Cold Vent } \\
\text { Establishment } \\
\text { Spillage Test }\end{array}$ & $\begin{array}{l}\text { Assess whether house depressurization } \\
\text { caused by air moving appliances in a house } \\
\text { could cause combustion appliance spillage, } \\
\text { compared to house specific norms. }\end{array}$ & $\begin{array}{l}\text { NFPA 54-1999, ASTM Guide E1998-99, } \\
\text { CAN/CGSB-51.71-95. }\end{array}$ & $\begin{array}{l}\text { Blower door or } \\
\text { equivalent and } \\
\text { digital pressure } \\
\text { sensor. }\end{array}$ & 180 & $\begin{array}{l}\text { Health and } \\
\text { Safety }\end{array}$ \\
\hline
\end{tabular}

\footnotetext{
* This test is not recommended, but is included for completeness because it is currently described in California Title 24 .
} 


\section{REFERENCES}

ASTM. 1994. “ASTM Standard E1554-94, Standard Test Methods for Determining External Air Leakage of Air Distribution Systems by Fan Pressurization”. Philadelphia, PA; American Society for Testing and Materials. http://www.astm.org.

ASTM. 1995. “ASTM Standard E1186-87, Standard Practices for Air Leakage Site Detection in Building Envelopes”. Philadelphia, PA; American Society for Testing and Materials.

http://www.astm.org.

ASTM. 1997. “ASTM Standard E1827-96, Standard Test Methods for Determining Airtightness of Buildings Using an Orifice Blower Door". Philadelphia, PA; American Society for Testing and Materials. http://www.astm.org.

ASTM. 1999a. "ASTM Standard E779-99, Standard Test Method for Determining Air Leakage Rate by Fan Pressurization”. Philadelphia, PA; American Society for Testing and Materials. http://www.astm.org.

ASTM. 1999b. "ASTM Guide E1998-99, Standard Guide For Assessing DepressurizationInduced Backdrafting and Spillage From Vented Combustion Appliances". Philadelphia, PA; American Society for Testing and Materials. http://www.astm.org.

CEE. 2000. "Specification of Energy-Efficient Installation and Maintenance Practices for Residential HVAC Systems”. Boston, MA; Consortium for Energy Efficiency. http://www.ceeformt.org/resid/rs-ac/hvac.php3.

CGSB. 1995. "CAN/CGSB-51.71-95, Method To Determine The Potential For Pressure-Induced Spillage From Vented, Fuel-Fired, Space Heating Appliances, Water Heaters and Fireplaces”. Ottawa, CA; Canadian General Standards Board. http://www.pwgsc.gc.ca/cgsb/catalogue/specs/051/051 071-e.html.

ConSol. 1999. “CIEE Final Project Report: Protocols for Energy Efficient Residential Building Envelopes". Stockton, CA; ConSol. Also available from the California Energy Commission at http://www.energy.ca.gov/efficiency/qualityhomes/protocols.html.

GAHI. 2000. "The GAHI Protocol for Exterior Insulation Finishing Systems (EIFS) Moisture Intrusion Inspections - One \& Two Family Homes”. Atlanta, GA: Georgia Association of Home Inspectors. http://www.gahi.com/eifsprot.html. November 1.

Lstiburek, J. and J. Carmody. 1994. "Moisture Control Handbook: Principles and Practices for Residential and Small Commercial Buildings". New York: Van Nostrand Reinhold. http://www.buildingscience.com/resources/books/default.htm.

NFPA. 1999. “NFPA 54-1999: National Fuel Gas Code”. Quincy, MA: National Fire Protection Association. http://www.nfpa.org/catalog/.

NHCID. 1998. "Moisture Testing Guide for Wood Frame Construction Clad with Exterior Insulation and Finish Systems (EIFS), Version 3.01". Prepared for EIFS Review Committee. Wilmington, NC: New Hanover County Inspection Department. August 4.

Palmiter, L. and P.W. Francisco. 2000. "Development of a Simple Device for Field Air Flow Measurement of Residential Air Handling Equipment: Phase II". Final Report of Ecotope, Inc. to the U.S. Department of Energy, STTR Grant \#DE-FG03-97ER86060. June. 
RDH. 2000. "Building Envelope Rehabilitation: Consultant Guide”. Report of RDH Building Engineering Limited to Canada Mortgage and Housing Corporation. Ottawa, ON: Canadian Housing Information Centre. April.

http://www.cmhc-schl.gc.ca/publications/en/rh-pr/tech/2000-115.htm.

Walker, I.S., M.H. Sherman, J. Wempen, D. Wang, J.A. McWilliams, and D.J. Dickerhoff, D.J. 2001. "Development of a New Duct Leakage Test: Delta Q". Lawrence Berkeley National Laboratory report LBNL-47308. http://epb1.1bl.gov/EPB/Publications/lbnl-47308.pdf.

Wray, C.P., I.S. Walker, J.A. Siegel, and M.H. Sherman. 2002. "Practical Diagnostics for Evaluating Residential Commissioning Metrics" Lawrence Berkeley National Laboratory report LBNL-45959. http://epb1.lbl.gov/EPB/Publications/lbnl-45959.pdf. 


\section{APPENDIX III. EXAMPLE COMMISSIONING REPORT}

Jennifer McWilliams, Craig Wray, Max Sherman, and Iain Walker

Lawrence Berkeley National Laboratory

Berkeley, California 


\section{Table III-1. Sample Commissioning Report}

The house surveyed is located in Concord, CA. It was built in 1976, and contains 2,462 square feet of living space. This house is a two-story, three-bedroom, two and a half bath home. The construction is wood frame with stucco siding, a clay tile roof, and an uninsulated slab-on-grade foundation. Below is a chart of the tests performed at this house and a summary of the results.

A visual inspection of the furnace/ air conditioner indicated that it is a 25 year old, low efficiency unit that is in need of replacement. There is an opportunity to replace this unit with a high efficiency (90\% AFUE) furnace that includes an evaporator equipped with TXV control and a 14 SEER A/C condenser. The new unit should be sized in accordance with the package of energy saving measures that are performed in the retrofit. Note that there is no need to increase the airhandler airflow or to add charge to the old cooling system if the unit is being replaced.

\begin{tabular}{|c|c|c|c|c|c|}
\hline Task & $\begin{array}{c}\text { Tuning \& } \\
\text { Tweaking (T) } \\
\text { or } \\
\text { Oportunity (O) }\end{array}$ & Name & Result & Recommendation & $\begin{array}{l}\text { Energy } \\
\text { Savings } \\
\text { Potential }\end{array}$ \\
\hline 1 & P & $\begin{array}{l}\text { Insulation } \\
\text { Inspection }\end{array}$ & $\begin{array}{l}\text { R-26 ceiling } \\
\text { R-11 walls } \\
\text { Uninsulated slab. }\end{array}$ & Add insulation in attic to bring it up to nominal R-38. & Medium \\
\hline 2 & $\mathrm{O}$ & $\begin{array}{l}\text { Window } \\
\text { Inspection }\end{array}$ & $\begin{array}{l}\text { Single-glazed aluminum } \\
\text { frame windows. }\end{array}$ & $\begin{array}{l}\text { Upgrade to double-glazed, low-e, low solar gain, fiberglass or } \\
\text { vinyl frame windows. }\end{array}$ & Medium \\
\hline 3 & $\mathrm{~T}$ & $\begin{array}{l}\text { Envelope } \\
\text { Airtightness Test } \\
\text { and Leak } \\
\text { Detection }\end{array}$ & $\begin{array}{l}\mathrm{NL}=0.97, \mathrm{ELA}=278 \mathrm{in}^{2} . \\
\text { Major leak sites were found } \\
\text { between the attic and living } \\
\text { spaces. }\end{array}$ & $\begin{array}{l}\text { Seal building envelope, but no tighter than } \mathrm{NL}=0.5 \text {. Seal } \\
\text { mechanical and plumbing chases in attic. Seal top of stairwell } \\
\text { walls in attic. Improve attic hatch weatherstripping. Seal } \\
\text { plumbing penetrations in kitchen and bathrooms. }\end{array}$ & Medium \\
\hline 5 & $\mathrm{~T}$ & $\begin{array}{l}\text { Delta Q Duct } \\
\text { Leakage Test }\end{array}$ & $\mathrm{Qs}=35 \mathrm{cfm}, \mathrm{Qr}=408 \mathrm{cfm}$ & $\begin{array}{l}\text { Install new return duct because the location of the existing duct } \\
\text { does not allow access for sealing. }\end{array}$ & High \\
\hline 7 & $\mathrm{~T}$ & $\begin{array}{l}\text { Air-Handler } \\
\text { Airflow: Fan- } \\
\text { Assisted Flow } \\
\quad \text { Meter }\end{array}$ & $804 \mathrm{cfm}$ & Increase airflow to $1200 \mathrm{cfm}$ if cooling system is not replaced. & Medium \\
\hline 11 & $\mathrm{~T}$ & $\begin{array}{l}\text { Supply Register } \\
\text { Airflows }\end{array}$ & $\begin{array}{l}\text { Refer to register flow chart } \\
\text { for measured values. }\end{array}$ & $\begin{array}{l}\text { Dining room airflow is } 25 \text { to } 35 \% \text { low, master bedroom airflow } \\
\text { is } 65 \% \text { low, other bedrooms and bathrooms are moderately } \\
\text { high. Adjust dampers to improve comfort. }\end{array}$ & Medium \\
\hline 12 & $\mathrm{~T}$ & $\begin{array}{c}\text { Superheat } \\
\text { Refrigerant } \\
\text { Charge Test }\end{array}$ & Low charge. & Add refrigerant charge if cooling system is not replaced. & High \\
\hline
\end{tabular}




\section{Table III-2. Supply Grille Airflow Comparison}

\begin{tabular}{|l|c|c|c|c|}
\hline Location of Grille & Measured flow [cfm] & Required flow* [cfm] & Deviation [cfm] & Deviation [\%] \\
\hline \hline Dining/living (near kitchen door) & 101 & 156 & $\mathbf{- 5 5}$ & $\mathbf{- 3 5}$ \\
\hline Dining/living (under piano) & 55 & 74 & -19 & -26 \\
\hline Game room (bathroom) & 126 & 131 & -5 & -4 \\
\hline Game room (laundry) & 140 & 131 & 9 & 7 \\
\hline Bathroom & 28 & 8 & 20 & 250 \\
\hline Master bedroom & 36 & 102 & $\mathbf{- 6 6}$ & $\mathbf{- 6 5}$ \\
\hline Master bedroom (closet) & 32 & 51 & -19 & -37 \\
\hline Master bathroom & 28 & 15 & 13 & 87 \\
\hline Bedroom (front of stairs) & 67 & 40 & 27 & 68 \\
\hline Bedroom (corner) & 60 & 45 & 15 & 33 \\
\hline Office & 76 & 43 & 33 & 77 \\
\hline Girls bathroom & 29 & 9 & 20 & 222 \\
\hline \hline Total & 778 & 805 & -27 & 3 \\
\hline \hline
\end{tabular}

* Required flow is based on room thermal loads calculated using ACCA Manual J for the post-retrofit condition. 